\title{
A Monte Carlo Evaluation of the Stock Synthesis Assessment Program
}

\author{
David B. Sampson and Yanshui Yin \\ Oregon State University, Newport, Oregon
}

\begin{abstract}
Stock assessments for many U.S. Pacific coast groundfish stocks are developed using the catch at age method known as Stock Synthesis. The Stock Synthesis computer program attempts to reconstruct the demographic history of a stock from observed changes in fish age or size distributions, coupled with auxiliary information such as an index of stock biomass developed from a research survey or an index of fishing mortality based on fishing effort. In this study Monte Carlo simulation techniques were used to generate fishery and survey data with known characteristics. The simulated data were then analyzed with the age-structured version of the Stock Synthesis program and results from the program were compared with the true values to evaluate the influence of measurement errors on the accuracy of the Stock Synthesis results. Data sets were constructed with low and high levels of random error in each of four types of sample data (fishery age composition, a fishing effort index, survey age composition, and a survey index of stock biomass). A series of experiments, based on a fractional factorial design, was conducted to examine the importance of eight factors: low versus high rates of natural mortality; constant versus variable annual recruitment; low versus high rates of increase in fishing mortality; dome-shaped versus asymptotic fishery selectivity; short versus long data series; low versus high variability in the fishing effort index; low versus high variability in the survey biomass index; and small versus large samples for age composition. On average the Stock Synthesis estimates for total biomass, exploitable biomass, recruitment, and fishing mortality in the ending year were slightly positively biased (3.5-6.1\%) but less variable than the input data. In general, the number of years in the data series and the size of the age samples were the most influential factors, with increased amounts of data producing less biased and less variable estimates.
\end{abstract}




\section{Introduction}

Although fisheries managers and the fishers they regulate generally appreciate that exploited fish stocks have limited productive capabilities and therefore cannot support unconstrained harvests, the fisheries scientists who advise the managers are rarely able to provide highly accurate estimates of the size or potential productivity of any stock. The patchiness and inaccessibility of most fishes, coupled with natural variability and an uncontrolled environment, make it particularly difficult to measure, let alone predict, the stock size and dynamics of wild fish populations. Inaccurate perceptions of stock size and dynamics can be costly, however. Estimates that are too small can result in lost fishing opportunities due to regulations that are too restrictive; estimates that are too liberal can result in overfishing, overcapitalization of the fishing fleet, and decreased long-term productivity of the fish stock.

Fisheries managers rely on fish stock assessments to provide them with a biological foundation for their management decisions. These assessments supply fundamental information about the status of the stocks, whether the stocks are increasing and can support increased levels of harvest, or whether they are decreasing and require stricter control of harvests. Often the stock assessments recommend annual catch quotas to act as upper bounds on the rates of fish removals. Although fisheries scientists are normally aware of the tremendous variability associated with fisheries data, stock assessment documents often do not state explicitly that the estimates of the current stock size can be highly inaccurate, and that recommended catch quotas may consequently fail to meet management objectives.

Determining a stock assessment's level of accuracy is a very involved process. In many modern stock assessments age-structured population models (Megrey 1989) are applied to catch at age data collected from multistage sampling schemes (Gavaris and Gavaris 1983, Quinn et al. 1983). It is a daunting challenge to estimate sampling variances for catch at age data. To trace the consequences of these sampling errors on the final stock assessment estimates is an even more formidable problem. However, there have been some attempts to do so (Pope and Garrod 1975, Mohn 1983, Rivard 1983, Sampson 1987, Prager and MacCall 1988, Kimura 1989, Rivard 1989, Pelletier and Gros 1991, Restrepo et al. 1992). If stock assessments could routinely provide estimates of their accuracy and measures of the relative importance of different sources of uncertainty, then it would be possible to identify which inputs were most in need of improvement. Appropriate modifications to the sampling programs could then be specified.

The stock assessments for many U.S. Pacific Coast groundfish stocks are developed using a catch at age method known as Stock Synthesis (Methot 1990, Pacific Fishery Management Council [PFMC] 1996). This program, which elaborates on the methods of Fournier and Archibald (1982) and 
Deriso et al. (1985), attempts to reconstruct the demographic history of a stock from observed changes in fish age distributions, coupled with auxiliary information such as survey indices of stock biomass. Stock Synthesis uses time series of catch biomass and age (or size) composition to derive maximum likelihood estimates of numerical stock abundance, stock biomass, and related parameters. It is a very powerful tool for examining fisheries data; it offers considerable flexibility in the types of data that it can accommodate.

In this study we evaluated the influence of random sampling errors in fishery and survey data on the accuracy of a suite of estimates output by the age-structured version of the Stock Synthesis assessment program. We used Monte Carlo simulation techniques (Rubinstein 1981) to generate fishery data with known characteristics. The random data sets were then analyzed using the Stock Synthesis program and estimates from Stock Synthesis were compared with the true values. There have been few published Monte Carlo evaluations of the Stock Synthesis program.

\section{Stock Synthesis Approach}

The Stock Synthesis program uses standard deterministic equations to describe the dynamics of an age-structured population (Methot 1990). The number of fish in a given year class follows an exponential decay function,

$$
N_{y a}=N_{y-1, a-1} \times \exp \left[-\left(M+S_{a-1} \times F_{y-1}\right)\right]
$$

where $N_{y a}$ denotes the number of fish at the start of year $y$ that are $a$ years old, $M$ is the instantaneous rate of natural mortality, $S_{a}$ is the selectivity coefficient for age $a$ fish, and $F_{y}$ is the instantaneous rate of fishing mortality in year $y$ for fully selected ages. For simplicity here, $M$ is constant and the $S_{a}$ do not vary from year to year. The number of fish in the oldest (terminal, $T$ ) age class is given by

$$
N_{y T}=N_{y-1, T} \times \exp \left[-\left(M+S_{T} \times F_{y-1}\right)\right]+N_{y-1, T-1} \times \exp \left[-\left(M+S_{T-1} \times F_{y-1}\right)\right]
$$

The Synthesis program hosts a range of methods for modeling the selectivity coefficients. We used the four parameter "double-logistic" function, which produces a selectivity versus age relationship that is sometimes described as being "dome-shaped,"

$$
S_{a}=\frac{\{1+\exp [-b 1 \times(a-a \mathrm{l})]\}^{-1} \times\{1+\exp [b 2 \times(a-a 2)]\}^{-1}}{\operatorname{Max} S},
$$

where $a$ c controls the inflection age and $b$ l controls the slope for the ascending portion of the curve, and $a 2$ and $b 2$ exert similar controls over 
the descending portion of the curve. MaxS, which is the maximum value of the numerator for integer values of age up to the terminal age, scales the selectivity coefficients so that at least one age class has a selectivity coefficient of one and thus suffers the full rate of fishing mortality. If parameter $b 2$ is zero, the selectivity function degenerates to a so-called "asymptotic" selectivity versus age relationship.

The catch at age (in numbers of fish) is given by the following catch equation,

$$
C_{y a}=\frac{N_{y a} S_{a} F_{y} \times\left\{1-\exp \left[-\left(M+S_{a} F_{y}\right)\right]\right\}}{M+S_{a} F_{y}} .
$$

The yield at age (catch in weight) is given by

$$
Y_{y a}=C_{y a} W_{a},
$$

where $W_{a}$ denotes the average weight of age $a$ fish in the fishery, here assumed to be time-independent.

As it is typically configured, the Synthesis program assumes that the data for catch biomass are measured with perfect accuracy and it adjusts the estimates for $F_{y}$ so that the estimate of total catch biomass,

$$
Y_{y}=\sum_{a} Y_{y a}
$$

equals the observed total catch biomass.

All methods for analyzing catch at age data require additional auxiliary information for tuning the analysis (Pope and Shepherd 1982, Shepherd and Nicholson 1991). It is otherwise impossible to distinguish a stock that is increasing from one that is decreasing. For tuning a Stock Synthesis analysis one can use survey indices of stock biomass or numerical abundance, or data series for fishing effort or catch per unit effort. If survey biomass data are used and the survey is conducted at the beginning of the year, the expected value of the survey biomass index is given by

$$
E\left[B_{y}^{\prime}\right]=Q^{\prime} \sum_{a} N_{y a} W_{a}^{\prime} S_{a}^{\prime},
$$

where $Q^{\prime}$ denotes the survey catchability coefficient, $W_{a}^{\prime}$ is the average fish weight at age in the survey, $S_{a}^{\prime}$ is the survey selectivity coefficient for age $a$ fish. If fishing effort data $(f)$ are used, the expected value of the effort is related to the rate of fishing mortality by

$$
F_{y}=Q E\left[f_{y}\right],
$$


where $Q$ denotes the fishery catchability coefficient.

The Stock Synthesis program is very flexible in allowing the user to either fix or estimate the various parameters that define the population structure and dynamics. For parameters that are estimated rather than prespecified, the values are maximum likelihood estimates and satisfy a total likelihood function of the form

$$
L_{\text {total }}=\sum_{i} L_{i} e_{i},
$$

where $L_{i}$ denotes the log-likelihood value for likelihood component $i$ and $e_{i}$ is the so-called "emphasis value" for component $i$. An application with the Synthesis program will include a likelihood component and emphasis value for each type of input data that contains observation error, e.g., the fishery age composition data, the fishing effort data, the survey age composition data, and the survey biomass index. If the entire model has been correctly defined, which includes properly specifying the measurement error associated with each type of observation, the emphasis values should all be equal to one.

By assuming that age determination is exact and that simple random samples of fish are obtained (either from the fishery or the survey), then the age composition data are distributed as multinomial random variables and the likelihood component for these data is

$$
L_{\text {age }}=\sum_{y} J_{y} \times \sum_{a}\left\{p_{y a} \times \log \left(E\left[p_{y a}\right]\right)-p_{y a} \times \log \left(p_{y a}\right)\right\}
$$

where $J_{y}$ is the number of fish in the sample for year $y, p_{y a}$ is the observed proportion at age in the sample for year $y$, and $E\left[p_{y a}\right]$ is the true proportion at age in the sample for year $y$. If the observed proportions at age are all equal to the expected proportions at age, the $L_{\text {age }}$ likelihood component will be zero.

If the survey estimates of biomass are distributed as a lognormally distributed random variable, then the likelihood component for these data is

$$
L_{\text {survey }}=-\log \left(\sigma_{S y}\right)-\sum_{y} \frac{1}{2 \sigma_{S y}^{2}} \times\left(\log \frac{B_{y}^{\prime}}{E\left[B_{y}^{\prime}\right]}\right)^{2},
$$

where $B_{y}^{\prime}$ is the observed survey estimate of biomass in year $y$ and $E\left[B_{y}^{\prime}\right]$ is its expected value, and $\sigma_{S y}$ is the true, log-scale standard deviation for these data,

$$
\sigma_{S y}=\sqrt{\log \left(1+c v_{S y}^{2}\right)}
$$


The term $c v_{s y}$ is the true, arithmetic-scale coefficient of variation of the survey biomass estimate in year $y$.

If the fishing effort data also follow a log-normal distribution, the likelihood component for these data is

$$
L_{\text {effort }}=-\log \left(\sigma_{F}\right)-\sum_{y} \frac{1}{2 \sigma_{F}^{2}} \times\left(\log \frac{f_{y}^{\prime}}{E\left[f_{y}^{\prime}\right]}\right)^{2},
$$

where $f_{y}^{\prime}$ is the observed fishing effort in year $y$ and $E\left[f_{y}^{\prime}\right]$ is its expected value, and $\sigma_{F}$ is the true, log-scale standard deviation for these data,

$$
\sigma_{F}=\sqrt{\log \left(1+c v_{F}^{2}\right)} .
$$

The term $c v_{F}$ is the true, arithmetic-scale coefficient of variation for the fishing effort series.

One can configure the Stock Synthesis program to include likelihood components that tend to constrain the estimates of annual recruitment to conform to a spawner recruit relationship. We specified zero emphasis on the spawner recruit components.

\section{Methods}

We used a brute-force approach for evaluating the performance of the Stock Synthesis program. We generated random data sets with known characteristics, analyzed the data sets using the Stock Synthesis program, and then compared estimates from Synthesis with the true values. We used the Windows 95 version of Stock Synthesis, which was provided to us in June 1997 by the program's author (Richard Methot, National Marine Fisheries Service, Northwest Fisheries Science Center, Seattle, WA). We produced the random data sets using two $\mathrm{C}++$ programs developed by Yanshui Yin. The first program simulates the dynamics of an age-structured population using the same deterministic equations (described above) that underlie Methot's Stock Synthesis program. The second program takes the true demographic data produced by the first program and generates random data sets that can be analyzed directly by the Stock Synthesis program.

\section{Stock Synthesis Configuration for this Study}

We simulated a simple fishery system: a single fishery with data available annually on total catch, age composition, and nominal fishing effort; and a single survey that provided estimates of annual stock biomass and age composition. In all cases we generated the simulated random data in such a manner that they would be unbiased, and we gave the Synthesis pro- 
gram the true parameter values as the initial values with which to begin its iterative search for the set of maximum likelihood parameter estimates. We conducted a small sensitivity study to examine the influence of using the true parameter values as the initial values. For all simulated stocks we generated a set of errorless data that we analyzed with Stock Synthesis and thereby verified an exact correspondence between the deterministic population equations of the data simulator and those of Stock Synthesis.

The Stock Synthesis program assumes that the data on catch biomass are exact, but in our simulations the catch biomass data were estimates that were lognormally distributed with a $10 \%$ relative accuracy (on the arithmetic scale). The age composition data were generated as simple random samples from the true catch and were not roughened to mimic randomness due to age-reading errors, nor were they distorted to mimic age-reading bias. The Stock Synthesis program was configured to treat the age composition data as being without reading error but with multinomial sampling error, and the program was given the true sample size. The fishing effort data for a given simulation were generated as lognormally distributed random variables with expected values equal to the true values $(F / Q)$ and with a fixed coefficient of variation $\left(c v_{F}\right)$ for all years. The Stock Synthesis program was configured to treat the effort data as being lognormally distributed and was given the true log-scale standard deviation for these data $\left(\sigma_{F}\right)$. The survey estimates of biomass for a given simulation were generated as lognormal random variables with expected values equal to the true values $\left(Q^{\prime} \times \sum_{a} N_{y a} W_{a}^{\prime} S_{a}^{\prime}\right)$ and with a fixed coefficient of variation $\left(c v_{S}\right)$ for all years. The Stock Synthesis program was configured to treat the survey biomass estimates as being lognormally distributed and was given the true $\log$-scale standard deviation for these data $\left(\sigma_{s}\right)$.

For each of the types of simulated data that were lognormally distributed (the catch biomass, fishing effort, and survey biomass) the mean and standard deviation on the arithmetic scale $(E[Y], V[Y])$ were related to the mean and standard deviation on the log scale $(\mu, \sigma)$ by the following:

$$
\begin{gathered}
E[Y]=\exp \left(\mu+0.5 \sigma^{2}\right), \\
V[Y]=\exp \left(2 \mu+\sigma^{2}\right) \times\left[\exp \left(\sigma^{2}\right)-1\right],
\end{gathered}
$$

i.e., the parameters defining lognormal random data were adjusted so that the generated random data did not have transformation bias.

The fish stock in all the simulations was unfished prior to the start of the simulated period and suffered an instantaneous rate of fishing mortality $(F)$ of $0.07 /$ yr during the first year, with $F$ increasing a fixed amount at the start of each year thereafter. Also, the instantaneous rate of natural mortality $(M)$ was constant with age and through time, and the Stock Synthesis program was configured with $M$ fixed at its correct value. The true 
fishery catchability coefficient and the survey catchability coefficient were constant throughout each simulated period $\left(E[Q]=0.003, E\left[Q^{\prime}\right]=0.1\right)$; the selectivity coefficients for the fishery and survey were also constant throughout each simulated period. There was no sexual dimorphism in the simulated stock. The two sexes had identical growth and identical vulnerability to the fishing and survey gear. The weight at age data were generated from the following deterministic equation,

$$
W_{a}=0.0001\{10[1-\exp (-0.2 a)]\}^{3}
$$

and the Synthesis program was given exact information on weight at age. In our simulations and in the Synthesis configuration weight at age changed abruptly with age at the start of each year.

\section{Experimental Design}

Random data sets were generated in accordance with a one-eighth fraction of a $2^{8}$ factorial design (Table 1). For each experimental treatment 200 random data sets were generated and then analyzed with Stock Synthesis. The eight control variables (and their low versus high levels) were: (1) the rate of natural mortality ( 0.2 versus $0.4 / \mathrm{yr})$; (2) the annual increase in the rate of fishing mortality $(0.01$ versus $0.02 / y r) ;(3)$ the number of years in the data series ( 8 versus 16 years); (4) annual recruitment (constant versus variable); (5) fishery selectivity (domed versus asymptotic); (6) the size of the annual age composition samples (100 versus 400 fish per annual sample); (7) the coefficient of variation of the annual fishing effort data (20\% versus $80 \%$, arithmetic scale); and (8) the coefficient of variation of the annual survey biomass estimates ( $20 \%$ versus $80 \%$, arithmetic scale).

Several stock parameters were coupled with the level of the natural mortality coefficient. When $M$ was $0.2 / y r$, the initial and terminal age classes were 4 and 20 years, the true ascending and descending inflection ages ( $a$ l and $a 2$ ) for the fishery selection function were 6 and 16 years, and the true ascending inflection age for the survey selection function was 5 years. When $M$ was $0.4 / y r$, the initial and terminal age classes were 2 and 10 years, the true ascending and descending inflection ages for the fishery selection function were 4 and 8 years, and the true ascending inflection age for the survey selection function was 3 years. The ascending slope coefficients $(b 1)$ for the fishery selection function and for the survey selection function were 1.0 and $1.5 / \mathrm{yr}$ for all simulated stocks. When the fishery selection function was domed, the descending slope coefficient (b2) was 1.0/yr.

For experimental treatments with constant recruitment, the annual recruitment was 3,000 fish (in thousands) and the initial age composition at the start of the first year was at equilibrium with this level of recruitment, and Stock Synthesis was configured to estimate the initial equilibri- 
Table 1. Fractional factorial experimental design.

Treatment FishSel NatMort RecVar FishMort NumYrs SmplSize FishCV SurvCV

\begin{tabular}{|c|c|c|c|c|c|c|c|c|}
\hline 1 & -1 & -1 & -1 & -1 & -1 & -1 & -1 & 1 \\
\hline 2 & 1 & -1 & -1 & -1 & -1 & 1 & 1 & 1 \\
\hline 3 & -1 & 1 & -1 & -1 & -1 & 1 & 1 & -1 \\
\hline 4 & 1 & 1 & -1 & -1 & -1 & -1 & -1 & -1 \\
\hline 5 & -1 & -1 & 1 & -1 & -1 & 1 & -1 & -1 \\
\hline 6 & 1 & -1 & 1 & -1 & -1 & -1 & 1 & -1 \\
\hline 7 & -1 & 1 & 1 & -1 & -1 & -1 & 1 & 1 \\
\hline 8 & 1 & 1 & 1 & -1 & -1 & 1 & -1 & 1 \\
\hline 9 & -1 & -1 & -1 & 1 & -1 & -1 & 1 & -1 \\
\hline 10 & 1 & -1 & -1 & 1 & -1 & 1 & -1 & -1 \\
\hline 11 & -1 & 1 & -1 & 1 & -1 & 1 & -1 & 1 \\
\hline 12 & 1 & 1 & -1 & 1 & -1 & -1 & 1 & 1 \\
\hline 13 & -1 & -1 & 1 & 1 & -1 & 1 & 1 & 1 \\
\hline 14 & 1 & -1 & 1 & 1 & -1 & -1 & -1 & 1 \\
\hline 15 & -1 & 1 & 1 & 1 & -1 & -1 & -1 & -1 \\
\hline 16 & 1 & 1 & 1 & 1 & -1 & 1 & 1 & -1 \\
\hline 17 & -1 & -1 & -1 & -1 & 1 & -1 & -1 & -1 \\
\hline 18 & 1 & -1 & -1 & -1 & 1 & 1 & 1 & -1 \\
\hline 19 & -1 & 1 & -1 & -1 & 1 & 1 & 1 & 1 \\
\hline 20 & 1 & 1 & -1 & -1 & 1 & -1 & -1 & 1 \\
\hline 21 & -1 & -1 & 1 & -1 & 1 & 1 & -1 & 1 \\
\hline 22 & 1 & -1 & 1 & -1 & 1 & -1 & 1 & 1 \\
\hline 23 & -1 & 1 & 1 & -1 & 1 & -1 & 1 & -1 \\
\hline 24 & 1 & 1 & 1 & -1 & 1 & 1 & -1 & -1 \\
\hline 25 & -1 & -1 & -1 & 1 & 1 & -1 & 1 & 1 \\
\hline 26 & 1 & -1 & -1 & 1 & 1 & 1 & -1 & 1 \\
\hline 27 & -1 & 1 & -1 & 1 & 1 & 1 & -1 & -1 \\
\hline 28 & 1 & 1 & -1 & 1 & 1 & -1 & 1 & -1 \\
\hline 29 & -1 & -1 & 1 & 1 & 1 & 1 & 1 & -1 \\
\hline 30 & 1 & -1 & 1 & 1 & 1 & -1 & -1 & -1 \\
\hline 31 & -1 & 1 & 1 & 1 & 1 & -1 & -1 & 1 \\
\hline 32 & 1 & 1 & 1 & 1 & 1 & 1 & 1 & 1 \\
\hline
\end{tabular}

Factors:

FishSel $=$ Fishery selectivity (domed vs. asymptotic).

NatMort = Natural mortality $(0.2$ vs. $0.4 /$ yr $)$.

RecVar = Recruitment variability (constant vs. variable).

FishMort $=$ Fishing mortality trend $(0.01$ vs. $0.02 / \mathrm{yr})$.

NumYrs $=$ Number of years of data ( 8 vs. 16 yrs).

SmplSize $=$ Sample size for age compositions (100 vs. 400 fish).

FishCV $=$ Fishing effort variability $(20 \%$ vs. $80 \%$ CV).

SurvCV $=$ Survey biomass variability (20\% vs. $80 \%$ CV). 
um age composition. For treatments with variable recruitment, the average annual recruitment was also 3,000 fish, but the annual recruitment values followed the sequence 3,500,4,000, 1,200,4,200,3,000,3,200, $1,700,3,200$ during the simulation period (with repetition as necessary) and for the years preceding the simulation, and Stock Synthesis was configured to estimate the initial non-equilibrium age composition.

Because we used a fractional factorial design, not all effects and interactions were separately estimable (Table 2). For example, the main effect for fishery selection was "aliased" with two four-way interactions and with two three-way interactions (natural mortality $\times$ recruitment variability $\times$ sample size) and (natural mortality $\times$ fishing mortality $\times$ effort variability), meaning that the value estimated for the fishery selectivity effect included these additional interactions (Box et al. 1978).

For any given data set the Stock Synthesis program produces a wide variety of outputs including ones for the annual series of biomass, fishing mortality, and recruitment. In our analyses we focused on the bias and variability of the Synthesis estimates for the last year for total biomass $\left(\sum N_{a} W_{a}\right)$, exploitable biomass $\left(\sum S_{a} N_{a} W_{a}\right)$, rate of fishing mortality, and recruitment, and on estimates for the first year for total biomass. To reduce potential non-normality of these outputs and yet provide replicates for subsequent analyses, for each experimental treatment and output type we separated the 200 sets of estimates from Synthesis into four groups of 50 and then calculated the relative bias and relative variability for each group. We measured relative bias within each group of 50 estimates using the average of

$$
\frac{(\text { estimated value-true value) }}{\text { true value }},
$$

and measured relative variability within each group using the coefficient of variation. To summarize results for each experimental treatment and output type we calculated the mean relative bias and mean coefficient of variation by averaging the four replicate measures. For each of the ten output types (the dependent variables) we conducted separate fractional analyses of variance using the Minitab statistics program (release 11.12 for Windows).

\section{Sensitivity to Initial Parameter Values}

Likelihood functions can have multiple maxima, in which case the choice of initial values for the parameters can influence whether or not the search algorithm finds a local rather than the global maximum. Also, when the search algorithm follows certain paths it may get stuck in regions where the likelihood surface is very flat. With each of the 200 replicates for each treatment in our main experiment we started the Stock Synthesis program with the true parameter values. To examine the influence of initial param- 
eter values on the performance of Stock Synthesis we generated 50 additional random data sets for two experimental treatments: treatment 6 , which had large relative variability in the output estimates; and treatment 30 , which had small relative variability. For each of these 100 data sets we ran the Synthesis program 20 times, each time using a different set of randomized initial parameter values, with each parameter varying uniformly within $\pm 50 \%$ of its true value.

\section{Results}

The five types of Stock Synthesis estimates that we examined varied greatly in relative bias and relative variability among the 32 experimental treatments (Table 3), with the estimates of ending exploitable biomass on average showing the largest negative bias $(-4.8 \%)$ and the estimates of the ending fishing mortality coefficient showing the largest positive bias (31.6\%). The mean relative variability ranged from a low of $3.6 \%$ for the estimates of starting biomass to a high of $92 \%$ for the estimates of the ending fishing mortality coefficient. For all 32 treatments the estimates of starting biomass were less variable than the other four types of estimates.

The Stock Synthesis estimates of ending biomass, ending exploitable biomass, ending recruitment, and starting biomass were in general skewed to the right, whereas the estimates of the ending fishing mortality coefficient were reasonably symmetric (e.g., Fig. 1). Because the analyses of variance were applied to averages of 50 values, the residuals from the analyses were reasonably well approximated by normal distributions. For the variables that measured relative bias, diagnostic plots of the residual versus fitted values indicated little evidence of heterogeneous variability, but similar plots for the variables that measured relative variation showed some tendency for residual variability to increase with the magnitude of the fitted values.

In the analyses of variance the main effects and two-way interactions were highly significant $(P<0.01)$ for all ten dependent variables (Table 4 ). The three-way interactions were not significant $(P<0.05)$ for any of the variables, however, except for the relative bias of the starting biomass estimate, which was significant at the $P=0.05$ level.

\section{Effects on Relative Bias}

On average across all levels of the eight factors the five types of estimates that we examined had slight but statistically significant $(P<0.05)$ positive bias, ranging from a low of $1.7 \%$ for the estimates of starting biomass to a high of $6.1 \%$ for the estimates of the ending fishing mortality coefficient (Table 5). The factor for age composition sample size was the most influential main effect for four of the five types of Synthesis estimates, and the estimated coefficients for this factor were negative for all five types of estimates, indicating that larger samples produced less biased estimates. 
Table 2. Alias structure of the fractional factorial design.

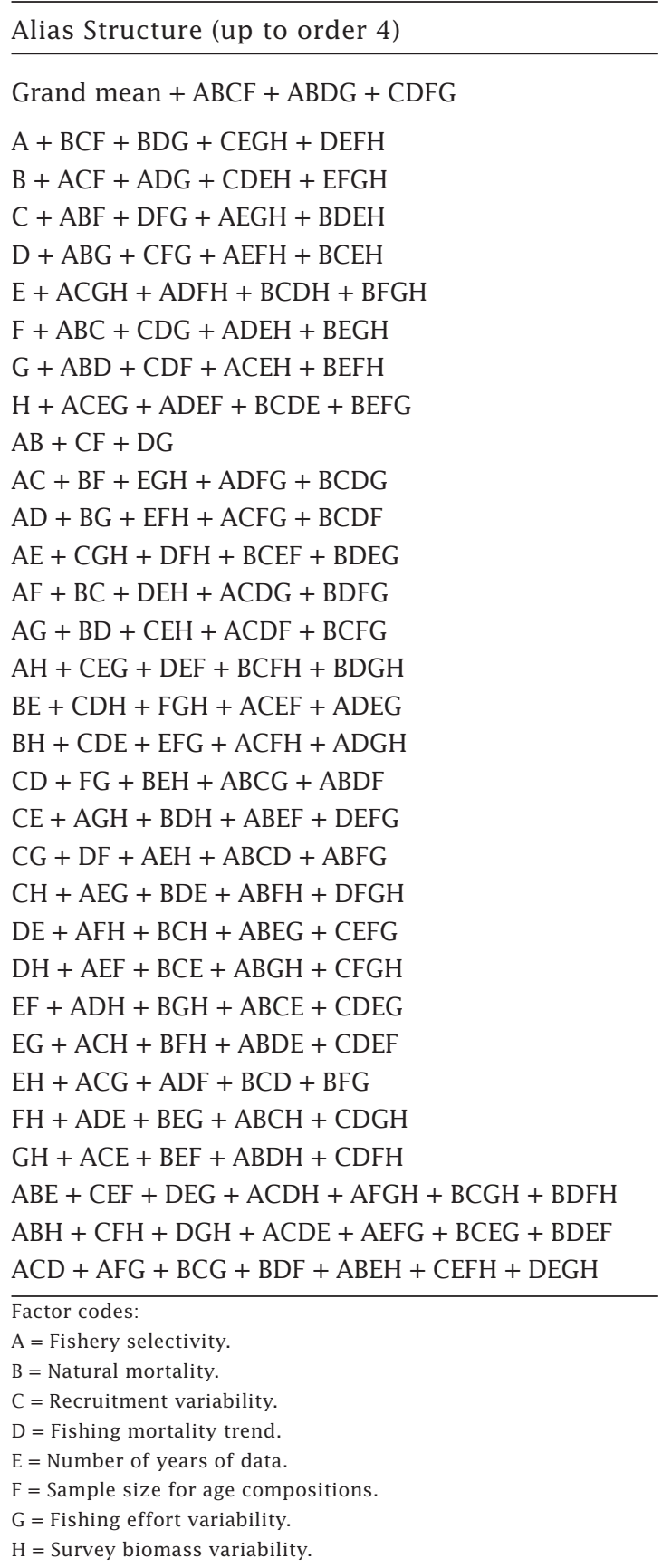


Table 3. Relative bias and relative variability for the 32 experimental treatments.

\begin{tabular}{|c|c|c|c|c|c|c|c|c|c|c|}
\hline \multirow[b]{2}{*}{ Treatment } & \multicolumn{5}{|c|}{ Mean relative bias } & \multicolumn{5}{|c|}{ Mean relative variability } \\
\hline & end Bio & end exB & end $F$ & end Rec & start Bio & end Bio & end exB & end $F$ & end Rec & start Bio \\
\hline 1 & 0.1015 & 0.0853 & 0.0159 & 0.1258 & 0.0557 & 0.3611 & 0.4201 & 0.3220 & 0.5804 & 0.2548 \\
\hline 2 & -0.0426 & -0.0436 & 0.2274 & -0.0260 & -0.0238 & 0.4029 & 0.3756 & 0.4961 & 0.4971 & 0.2019 \\
\hline 3 & 0.0439 & 0.0468 & 0.0121 & 0.0411 & 0.0338 & 0.2124 & 0.2282 & 0.2986 & 0.2522 & 0.1670 \\
\hline 4 & 0.0775 & 0.0796 & 0.0493 & 0.0990 & 0.0487 & 0.3731 & 0.4292 & 0.3495 & 0.5258 & 0.2743 \\
\hline 5 & -0.0001 & -0.0027 & 0.0463 & -0.0050 & -0.0037 & 0.2135 & 0.2156 & 0.2592 & 0.2867 & 0.1330 \\
\hline 6 & 0.1659 & 0.1571 & 0.0321 & 0.2094 & 0.0825 & 0.4972 & 0.5871 & 0.4280 & 0.7682 & 0.3283 \\
\hline 7 & -0.0242 & -0.0222 & 0.3160 & -0.0023 & -0.0225 & 0.4011 & 0.4002 & 0.9220 & 0.4869 & 0.2914 \\
\hline 8 & -0.0401 & -0.0483 & 0.1472 & -0.0337 & -0.0279 & 0.2827 & 0.2615 & 0.3504 & 0.3398 & 0.1447 \\
\hline 9 & 0.0991 & 0.0959 & 0.0241 & 0.1080 & 0.0585 & 0.3212 & 0.3775 & 0.3486 & 0.5367 & 0.2068 \\
\hline 10 & 0.0628 & 0.0602 & 0.0019 & 0.0872 & 0.0282 & 0.2699 & 0.2810 & 0.2575 & 0.4018 & 0.1285 \\
\hline 11 & 0.0197 & 0.0204 & 0.0360 & 0.0257 & 0.0138 & 0.2106 & 0.2194 & 0.2658 & 0.2720 & 0.1294 \\
\hline 12 & 0.0485 & 0.0380 & 0.2878 & 0.0915 & 0.0166 & 0.4852 & 0.4941 & 0.7910 & 0.6657 & 0.2405 \\
\hline 13 & 0.0633 & 0.0589 & 0.0136 & 0.0796 & 0.0370 & 0.2773 & 0.2943 & 0.3112 & 0.3949 & 0.1599 \\
\hline 14 & 0.1414 & 0.1261 & 0.0345 & 0.1725 & 0.0580 & 0.4643 & 0.5370 & 0.3863 & 0.6951 & 0.2556 \\
\hline 15 & -0.0061 & -0.0069 & 0.1290 & 0.0274 & -0.0011 & 0.3341 & 0.3311 & 0.4285 & 0.4226 & 0.2774 \\
\hline 16 & 0.0421 & 0.0434 & 0.0292 & 0.0563 & 0.0227 & 0.2832 & 0.2920 & 0.2880 & 0.3514 & 0.1498 \\
\hline 17 & 0.0141 & 0.0009 & 0.0135 & 0.0279 & 0.0044 & 0.1461 & 0.1490 & 0.1702 & 0.3349 & 0.0743 \\
\hline 18 & 0.0094 & 0.0108 & 0.0183 & -0.0048 & 0.0047 & 0.1744 & 0.1705 & 0.2129 & 0.2506 & 0.0426 \\
\hline 19 & 0.0236 & 0.0236 & 0.0467 & 0.0271 & 0.0027 & 0.2706 & 0.2763 & 0.3148 & 0.3379 & 0.0948 \\
\hline 20 & 0.0295 & 0.0255 & 0.0257 & 0.0768 & 0.0050 & 0.2349 & 0.2466 & 0.2371 & 0.3963 & 0.1155 \\
\hline 21 & 0.0070 & 0.0024 & 0.0098 & 0.0284 & 0.0036 & 0.1557 & 0.1608 & 0.1720 & 0.2409 & 0.0597 \\
\hline 22 & 0.1275 & 0.1139 & 0.1960 & 0.1720 & 0.0178 & 0.5265 & 0.5542 & 0.7680 & 0.8750 & 0.0936 \\
\hline 23 & 0.0508 & 0.0623 & 0.0178 & 0.0607 & 0.0732 & 0.2557 & 0.2753 & 0.3092 & 0.3467 & 0.2243 \\
\hline 24 & 0.0246 & 0.0228 & 0.0034 & 0.0318 & 0.0014 & 0.1381 & 0.1466 & 0.1667 & 0.1896 & 0.0647 \\
\hline 25 & -0.0046 & -0.0111 & 0.0884 & 0.0060 & -0.0037 & 0.2575 & 0.2635 & 0.3053 & 0.4238 & 0.0591 \\
\hline 26 & 0.0165 & 0.0149 & 0.0043 & 0.0178 & 0.0044 & 0.1625 & 0.1575 & 0.1698 & 0.2286 & 0.0358 \\
\hline 27 & 0.0184 & 0.0197 & -0.0121 & 0.0275 & 0.0056 & 0.1443 & 0.1534 & 0.1623 & 0.1920 & 0.0754 \\
\hline 28 & 0.0170 & 0.0269 & 0.0132 & 0.0041 & 0.0127 & 0.1773 & 0.2013 & 0.2502 & 0.2895 & 0.0825 \\
\hline 29 & 0.0054 & 0.0014 & 0.0159 & 0.0093 & 0.0021 & 0.1314 & 0.1338 & 0.1873 & 0.2078 & 0.0423 \\
\hline 30 & 0.0287 & 0.0237 & 0.0110 & 0.0690 & 0.0044 & 0.1259 & 0.1260 & 0.1605 & 0.3254 & 0.0463 \\
\hline 31 & 0.0092 & 0.0218 & 0.0578 & 0.0103 & 0.0357 & 0.2463 & 0.2601 & 0.3038 & 0.3238 & 0.1811 \\
\hline 32 & 0.0839 & 0.0831 & 0.0252 & 0.1040 & 0.0044 & 0.3068 & 0.3278 & 0.3553 & 0.4042 & 0.0526 \\
\hline Min & -0.0426 & -0.0483 & -0.0121 & -0.0337 & -0.0279 & 0.1259 & 0.1260 & 0.1605 & 0.1896 & 0.0358 \\
\hline Max & 0.1659 & 0.1571 & 0.3160 & 0.2094 & 0.0825 & 0.5265 & 0.5871 & 0.9220 & 0.8750 & 0.3283 \\
\hline
\end{tabular}


Ending Biomass

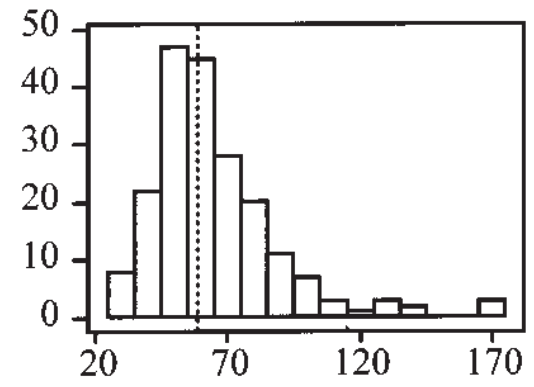

Ending Recruitment

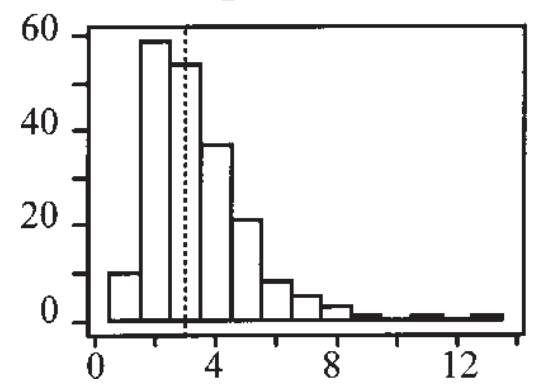

Ending F

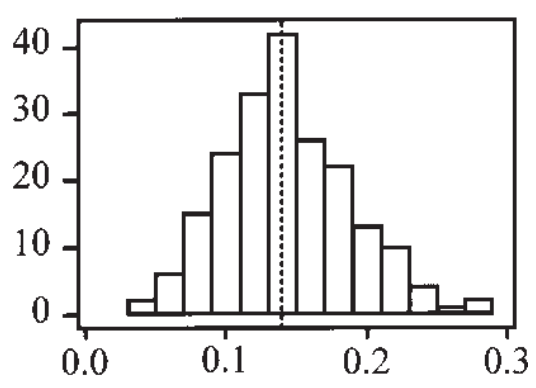

Starting Biomass

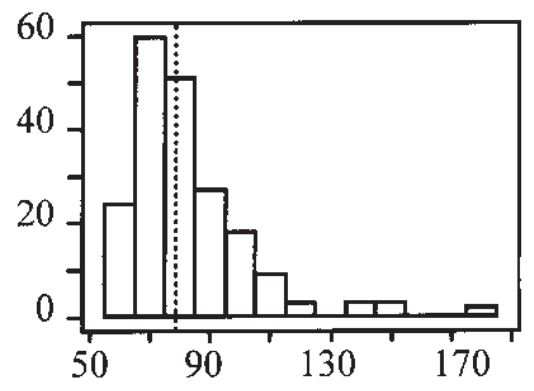

Figure 1. Example histograms from experimental treatment 1 of variables output by the Stock Synthesis program and used as dependent experimental variables. The dashed vertical lines indicate the true values. The units for the biomass and recruitment axes are in thousands. 
The number of years in the data series was the only other factor that produced a significant $(P<0.05)$ main effect for all five types of estimates, and in all cases an increase in the number of years produced less biased estimates. For the estimates of ending biomass, ending exploitable biomass, and ending recruitment, however, the interactions between these two factors were significantly $(P<0.05)$ positive, indicating that these two effects were not additive. Recruitment variability was the only factor that did not produce a significant $(P<0.05)$ main effect for any of the estimate types, but there were significant interactions with this factor for several estimate types. For all five estimate types there were significant $(P<0.05)$ interactions between the natural mortality coefficient and the number of years, between the recruitment variability and the fishing effort variability, and between the number of years and the survey biomass variability.

\section{Effects on Relative Variability}

The overall average relative variability of the five types of Stock Synthesis estimates ranged from a low of $14.6 \%$ for the estimates of starting biomass to a high of $40.1 \%$ for the estimates of ending recruitment (Table 6). The factors for the number of years in the data series and for the age composition sample size produced the first or second most influential main effects for all five types of estimates, and the estimated coefficients for these factors were always negative, indicating that larger samples and longer data series produced less variable estimates. The interactions between these two factors were significantly $(P<0.05)$ positive in all cases, however, indicating that these two effects were not additive. The factors for fishing effort variability and survey biomass variability were significant $(P<0.05)$ and had great influence over the variability in the estimates of ending biomass, ending exploitable biomass, ending fishing mortality, and ending recruitment, but not over the estimate of starting biomass. All eight factors produced significant $(P<0.05)$ main effects for at least three of the five types of estimates.

\section{Sensitivity to Initial Parameter Values}

For treatment 30, which in the main experiment produced output values with little variability, using randomized initial parameter values had essentially no effect on the final Stock Synthesis output values. For example, across the 50 random data sets the coefficients of variation for the estimates of ending biomass (calculated from 20 replicates for each data set) ranged from $0.06 \%$ to $0.26 \%$ and the coefficients of variation for the log-likelihood values ranged from less than $0.0001 \%$ to $0.14 \%$. For treatment 6 , however, which in the main experiment produced output values with large variability, the Synthesis program did not always converge to the same output values when started with randomized parameter values. Although the final log-likelihood values were consistent across the 50 ran- 
Table 4. ANOVA tables from fractional factorial experiment.

\begin{tabular}{lrcccc}
\hline Source & DF & SS & MS & $F$ & $P$ \\
\hline Relative bias in ending total biomass. & & & & \\
Main effects & 8 & 0.0916 & 0.0114 & 7.08 & 0.000 \\
2-way interactions & 20 & 0.2066 & 0.0103 & 6.39 & 0.000 \\
3-way interactions & 3 & 0.0065 & 0.0022 & 1.33 & 0.269 \\
Residual error & 96 & 0.1552 & 0.0016 & & \\
Total & 127 & 0.4599 & & &
\end{tabular}

Relative bias in ending exploitable biomass.

$\begin{array}{lrllll}\text { Main effects } & 8 & 0.0757 & 0.0095 & 5.73 & 0.000 \\ \text { 2-way interactions } & 20 & 0.1967 & 0.0098 & 5.96 & 0.000 \\ \text { 3-way interactions } & 3 & 0.0051 & 0.0017 & 1.03 & 0.383 \\ \text { Residual error } & 96 & 0.1584 & 0.0017 & & \\ \text { Total } & 127 & 0.4359 & & & \end{array}$

Relative bias in ending $F$.

$\begin{array}{lrrrrr}\text { Main effects } & 8 & 0.4512 & 0.0564 & 19.18 & 0.000 \\ \text { 2-way interactions } & 20 & 0.4188 & 0.0209 & 7.12 & 0.000 \\ \text { 3-way interactions } & 3 & 0.0109 & 0.0036 & 1.23 & 0.303 \\ \text { Residual error } & 96 & 0.2822 & 0.0029 & & \\ \text { Total } & 127 & 1.1631 & & & \end{array}$

Relative bias in ending recruitment.

\begin{tabular}{lrrrrr} 
Main effects & 8 & 0.1669 & 0.0209 & 8.23 & 0.000 \\
2-way interactions & 20 & 0.2526 & 0.0126 & 4.98 & 0.000 \\
3-way interactions & 3 & 0.0119 & 0.0040 & 1.56 & 0.203 \\
Residual error & 96 & 0.2434 & 0.0025 & & \\
Total & 127 & 0.6748 & & & \\
Relative bias in starting biomass. & & & & \\
Main effects & 8 & 0.0269 & 0.0034 & 7.37 & 0.000 \\
2-way interactions & 20 & 0.0603 & 0.0030 & 6.62 & 0.000 \\
3-way interactions & 3 & 0.0045 & 0.0015 & 3.29 & 0.024 \\
Residual error & 96 & 0.0437 & 0.0005 & & \\
Total & 127 & 0.1354 & & & \\
\hline
\end{tabular}


Table 4. (Continued.)

\begin{tabular}{lrrrrr}
\hline Source & DF & SS & MS & $F$ & $P$ \\
\hline Relative variability in ending total biomass. & & & & \\
Main effects & 8 & 1.2957 & 0.1620 & 59.14 & 0.000 \\
2-way interactions & 20 & 0.3174 & 0.0159 & 5.80 & 0.000 \\
3-way interactions & 3 & 0.0091 & 0.0030 & 1.11 & 0.350 \\
Residual error & 96 & 0.2629 & 0.0027 & & \\
Total & 127 & 1.8851 & & &
\end{tabular}

Relative variability in ending exploitable biomass.

$\begin{array}{lrrrrr}\text { Main effects } & 8 & 1.5774 & 0.1972 & 47.57 & 0.000 \\ \text { 2-way interactions } & 20 & 0.4643 & 0.0232 & 5.60 & 0.000 \\ \text { 3-way interactions } & 3 & 0.0102 & 0.0034 & 0.82 & 0.485 \\ \text { Residual error } & 96 & 0.3979 & 0.0041 & & \\ \text { Total } & 127 & 2.4498 & & & \end{array}$

Relative variability in ending $F$.

\begin{tabular}{lrrrrr} 
Main effects & 8 & 2.8859 & 0.3607 & 39.13 & 0.000 \\
2-way interactions & 20 & 1.2001 & 0.0600 & 6.51 & 0.000 \\
3-way interactions & 3 & 0.0485 & 0.0162 & 1.75 & 0.161 \\
Residual error & 96 & 0.8850 & 0.0092 & & \\
Total & 127 & 5.0195 & & & \\
Relative variability in ending R. & & & & \\
Main effects & 8 & 2.8630 & 0.3579 & 52.36 & 0.000 \\
2-way interactions & 20 & 0.6966 & 0.0348 & 5.10 & 0.000 \\
3-way interactions & 3 & 0.0280 & 0.0093 & 1.36 & 0.259 \\
Residual error & 96 & 0.6561 & 0.0068 & & \\
Total & 127 & 4.2437 & & & \\
Relative variability in starting & biomass. & & & \\
Main effects & 8 & 0.7888 & 0.0986 & 68.17 & 0.000 \\
2-way interactions & 20 & 0.1335 & 0.0067 & 4.62 & 0.000 \\
3-way interactions & 3 & 0.0010 & 0.0003 & 0.22 & 0.880 \\
Residual error & 96 & 0.1388 & 0.0014 & & \\
Total & 127 & 1.0621 & & & \\
\hline
\end{tabular}


Table 5. Analysis of relative bias.

\begin{tabular}{|c|c|c|c|c|c|}
\hline Factor & End Bio & End exBio & End $F$ & End Rec & Start Bio \\
\hline Grand mean & $0.03792^{\mathrm{a}}$ & $0.00359^{a}$ & $0.06055^{\mathrm{a}}$ & $0.05388^{a}$ & $0.01734^{a}$ \\
\hline FishSel & $0.01163^{a}$ & $0.01055^{a}$ & 0.00862 & $0.01655^{a}$ & -0.00111 \\
\hline NatMort & $-0.01178^{a}$ & $-0.00806^{\mathrm{a}}$ & $0.01346^{a}$ & $-0.01343^{a}$ & -0.00329 \\
\hline RecVar & 0.00453 & 0.00447 & 0.00726 & 0.00797 & 0.00063 \\
\hline FishMort & 0.00240 & 0.00319 & $-0.01305^{a}$ & 0.00212 & 0.00137 \\
\hline NumYrs & $-0.00911^{\mathrm{a}}$ & $-0.00766^{\mathrm{a}}$ & $-0.02711^{\mathrm{a}}$ & $-0.01214^{a}$ & $-0.00619^{a}$ \\
\hline SmplSize & $-0.01682^{\mathrm{a}}$ & $-0.01572^{\mathrm{a}}$ & $-0.02146^{a}$ & $-0.02475^{a}$ & $-0.01053^{a}$ \\
\hline FishCV & 0.00639 & $0.00750^{\mathrm{a}}$ & $0.02470^{\mathrm{a}}$ & 0.00461 & 0.00258 \\
\hline SurvCV & -0.00292 & -0.00478 & $0.03523^{a}$ & 0.00084 & $-0.00629^{a}$ \\
\hline FishSel×NatMort & -0.00238 & -0.00396 & $-0.01001^{\mathrm{a}}$ & -0.00327 & -0.00249 \\
\hline FishSel×RecVar & $0.01768^{\mathrm{a}}$ & $0.01487^{\mathrm{a}}$ & $-0.01659^{a}$ & $0.01927^{\mathrm{a}}$ & 0.00355 \\
\hline FishSel×FishMort & 0.00317 & 0.00297 & -0.00521 & 0.00275 & 0.00132 \\
\hline FishSel×NumYrs & 0.00171 & 0.00199 & -0.00492 & 0.00055 & -0.00319 \\
\hline FishSel×SmplSize & $-0.01316^{\mathrm{a}}$ & $-0.01225^{a}$ & 0.00942 & $-0.01662^{\mathrm{a}}$ & $-0.00394^{\mathrm{a}}$ \\
\hline FishSel×FishCV & 0.00054 & 0.00032 & $0.00980^{\mathrm{a}}$ & 0.00077 & -0.00162 \\
\hline FishSel $\times$ SurvCV & -0.00104 & -0.00239 & $0.01413^{a}$ & 0.00060 & -0.00314 \\
\hline NatMort×NumYrs & $0.01508^{a}$ & $0.01610^{\mathrm{a}}$ & $-0.02471^{\mathrm{a}}$ & $0.01448^{\mathrm{a}}$ & $0.00974^{\mathrm{a}}$ \\
\hline NatMort $\times$ SurvCV & -0.00446 & -0.00477 & 0.00855 & -0.00386 & $-0.00428^{a}$ \\
\hline RecVar×FishMort & 0.00113 & 0.00095 & $-0.01522^{\mathrm{a}}$ & 0.00207 & 0.00106 \\
\hline RecVar $\times$ NumYrs & $0.00880^{\mathrm{a}}$ & $0.00929^{a}$ & 0.00142 & $0.01096^{\mathrm{a}}$ & $0.00603^{\mathrm{a}}$ \\
\hline RecVar $\times$ FishCV & $0.01549^{\mathrm{a}}$ & $0.01494^{\mathrm{a}}$ & $-0.01177^{a}$ & $0.01967^{\mathrm{a}}$ & $0.00659^{\mathrm{a}}$ \\
\hline RecVar $\times$ SurvCV & 0.00647 & 0.00695 & -0.00301 & 0.00367 & 0.00157 \\
\hline FishMort×NumYrs & $-0.00941^{\mathrm{a}}$ & $-0.00831^{\mathrm{a}}$ & 0.00508 & $-0.01287^{a}$ & $-0.00431^{a}$ \\
\hline FishMort×SurvCV & $0.00982^{\mathrm{a}}$ & $0.01029^{a}$ & $-0.01427^{a}$ & 0.00658 & $0.00836^{\mathrm{a}}$ \\
\hline NumYrs $\times$ SmplSize & $0.01159^{a}$ & $0.01040^{\mathrm{a}}$ & 0.00197 & $0.01314^{\mathrm{a}}$ & 0.00299 \\
\hline NumYrs $\times$ FishCV & 0.00392 & 0.00371 & -0.00544 & 0.00094 & 0.00051 \\
\hline NumYrs $\times$ SurvCV & $0.01068^{\mathrm{a}}$ & $0.01139^{a}$ & $-0.01193^{a}$ & $0.01273^{a}$ & $0.00389^{a}$ \\
\hline SmplSize×SurvCV & -0.00179 & -0.00090 & $-0.01053^{a}$ & -0.00212 & 0.00126 \\
\hline FishCV×SurvCV & -0.00697 & $-0.00796^{\mathrm{a}}$ & $0.02967^{\mathrm{a}}$ & -0.00284 & $-0.01007^{\mathrm{a}}$ \\
\hline FishSel $\times$ NatMort $\times$ NumYrs & -0.00432 & -0.00472 & 0.00097 & -0.00244 & $-0.00492^{\mathrm{a}}$ \\
\hline FishSel×NatMort $\times$ SurvCV & 0.00350 & 0.00264 & -0.00906 & 0.00835 & 0.00278 \\
\hline FishSel $\times$ RecVar $\times$ FishMort & -0.00442 & -0.00325 & -0.00136 & -0.00415 & -0.00179 \\
\hline Coefficient Std Deviation & 0.00355 & 0.00359 & 0.00479 & 0.00445 & 0.00189 \\
\hline
\end{tabular}

Factor coefficients (relative bias) from fractional factorial experiment.

a Coefficients with $t$-statistics significant at the $P=0.05$ level. 
Table 6. Analysis of relative variability.

\begin{tabular}{|c|c|c|c|c|c|}
\hline Factor & End Bio & End exBio & End $F$ & End Rec & Start Bio \\
\hline Grand mean & $0.27637^{a}$ & $0.29207^{a}$ & $0.33586^{a}$ & $0.40137^{a}$ & $0.14649^{a}$ \\
\hline FishSel & $0.03020^{\mathrm{a}}$ & $0.03216^{\mathrm{a}}$ & $0.01834^{\mathrm{a}}$ & $0.04887^{\mathrm{a}}$ & -0.00543 \\
\hline NatMort & -0.00410 & -0.00813 & $0.02620^{\mathrm{a}}$ & $-0.03911^{\mathrm{a}}$ & $0.01385^{\mathrm{a}}$ \\
\hline RecVar & $0.01362^{\mathrm{a}}$ & $0.01437^{a}$ & $0.02638^{\mathrm{a}}$ & $0.01480^{\mathrm{a}}$ & $0.01004^{\mathrm{a}}$ \\
\hline FishMort & $-0.01401^{\mathrm{a}}$ & $-0.01398^{a}$ & $-0.02513^{a}$ & $-0.01792^{\mathrm{a}}$ & $-0.01380^{\mathrm{a}}$ \\
\hline NumYrs & $-0.06049^{a}$ & $-0.06691^{a}$ & $-0.07054^{\mathrm{a}}$ & $-0.06595^{a}$ & $-0.06246^{\mathrm{a}}$ \\
\hline SmplSize & $-0.04911^{\mathrm{a}}$ & $-0.06119^{a}$ & $-0.06913^{a}$ & $-0.09841^{\mathrm{a}}$ & $-0.04137^{\mathrm{a}}$ \\
\hline FishCV & $0.03492^{\mathrm{a}}$ & $0.03615^{a}$ & $0.07578^{\mathrm{a}}$ & $0.04165^{\mathrm{a}}$ & 0.00585 \\
\hline SurvCV & $0.03901^{\mathrm{a}}$ & $0.03597^{a}$ & $0.06854^{\mathrm{a}}$ & $0.04626^{a}$ & 0.00165 \\
\hline FishSel $\times$ NatMort & $-0.01730^{\mathrm{a}}$ & $-0.01622^{\mathrm{a}}$ & $-0.03185^{a}$ & $-0.01585^{a}$ & $-0.01434^{\mathrm{a}}$ \\
\hline FishSel $\times$ RecVar & 0.00791 & $0.01538^{a}$ & $-0.01771^{\mathrm{a}}$ & $0.02852^{\mathrm{a}}$ & $-0.00918^{a}$ \\
\hline FishSel $\times$ FishMort & -0.00817 & -0.00820 & 0.00329 & -0.01212 & -0.00333 \\
\hline FishSel×NumYrs & $-0.01526^{a}$ & $-0.01601^{\mathrm{a}}$ & 0.00637 & -0.01442 & $-0.01192^{\mathrm{a}}$ \\
\hline FishSel×SmplSize & -0.00489 & $-0.01150^{\mathrm{a}}$ & 0.00200 & $-0.01895^{a}$ & 0.00286 \\
\hline FishSel×FishCV & $0.01522^{\mathrm{a}}$ & $0.01494^{\mathrm{a}}$ & $0.01871^{\mathrm{a}}$ & $0.02079^{a}$ & 0.00205 \\
\hline FishSel×SurvCV & $0.01266^{\mathrm{a}}$ & 0.00905 & $0.02149^{\mathrm{a}}$ & $0.01619^{a}$ & -0.00020 \\
\hline NatMort $\times$ NumYrs & $0.00996^{a}$ & $0.01889^{a}$ & $-0.02911^{\mathrm{a}}$ & 0.01367 & $0.01349^{a}$ \\
\hline NatMort $\times$ SurvCV & -0.00651 & -0.00917 & 0.01191 & -0.00521 & -0.00575 \\
\hline RecVar $\times$ FishMort & -0.00482 & -0.00474 & $-0.03449^{a}$ & -0.00760 & 0.00289 \\
\hline RecVar×NumYrs & 0.00631 & 0.00854 & 0.01112 & 0.01393 & 0.00150 \\
\hline RecVar $\times$ FishCV & $0.00998^{\mathrm{a}}$ & $0.01548^{\mathrm{a}}$ & 0.00804 & $0.02154^{\mathrm{a}}$ & 0.00539 \\
\hline RecVar×SurvCV & 0.00359 & 0.00702 & 0.01527 & 0.00762 & -0.00337 \\
\hline FishMort $\times$ NumYrs & -0.00787 & -0.00827 & -0.00336 & $-0.01812^{\mathrm{a}}$ & 0.00166 \\
\hline FishMort×SurvCV & -0.00007 & 0.00511 & $-0.01817^{\mathrm{a}}$ & -0.00370 & 0.00491 \\
\hline NumYrs $\times$ SmplSize & $0.01870^{\mathrm{a}}$ & $0.02685^{\mathrm{a}}$ & $0.02144^{\mathrm{a}}$ & $0.01943^{a}$ & $0.01582^{\mathrm{a}}$ \\
\hline NumYrs $\times$ FishCV & $0.01172^{\mathrm{a}}$ & $0.01401^{\mathrm{a}}$ & -0.00326 & $0.01483^{a}$ & -0.00341 \\
\hline NumYrs $\times$ SurvCV & $0.01522^{\mathrm{a}}$ & $0.01971^{\mathrm{a}}$ & -0.00564 & $0.02210^{\mathrm{a}}$ & 0.00084 \\
\hline SmplSize×SurvCV & -0.00764 & -0.00771 & $-0.03087^{a}$ & -0.00980 & 0.00306 \\
\hline FishCV $\times$ SurvCV & $0.01569^{a}$ & 0.00904 & $0.05275^{a}$ & $0.02138^{a}$ & -0.00477 \\
\hline FishSel $\times$ NatMort $\times$ NumYrs & -0.00509 & -0.00528 & -0.00296 & -0.00871 & -0.00086 \\
\hline FishSel $\times$ NatMort $\times$ SurvCV & -0.00292 & -0.00325 & $-0.01699^{a}$ & -0.00104 & 0.00204 \\
\hline FishSel $\times$ RecVar $\times$ FishMort & -0.00605 & -0.00644 & -0.00901 & -0.01190 & -0.00164 \\
\hline Coefficient Std Deviation & 0.00463 & 0.00569 & 0.00849 & 0.00731 & 0.00336 \\
\hline
\end{tabular}

Factor coefficients (relative variability) from fractional factorial experiment.

a Coefficients with $t$-statistics significant at the $P=0.05$ level. 
dom data sets, with coefficients of variation ranging from $0.0013 \%$ to $0.28 \%$, the estimates of ending biomass were sometimes quite variable, with coefficients of variation ranging from $0.71 \%$ to $27 \%$. Scatterplots of the ending biomass estimates versus the log-likelihood values (e.g., Fig. 2) indicated that the likelihood functions for these data sets were flat and did not have well-defined maxima; the Synthesis program sometimes stopped prematurely. However, we saw no evidence of likelihood functions having multiple maxima.

Also, when the Synthesis program started with the true parameter values for the 50 data sets from treatment 30 , it produced final ending biomass estimates that were within $-0.49 \%$ to $0.52 \%$ of the estimates associated with the observed maximum likelihood values; for 44 of the data sets the final ending biomass estimates were within the observed ranges of estimates produced by starting with random initial parameter values (20 replicates per data set). When the Synthesis program started with the true parameter values for the 50 data sets from treatment 6 , it produced final ending biomass estimates that were within $-25 \%$ to $8.7 \%$ of the observed maximum likelihood estimates; for 44 of the 50 data sets the final ending biomass estimates were within the observed ranges produced by starting with random initial parameter values. Hence, it appears that the results in the main experiment would not have differed substantially had we used a different strategy for choosing sets of initial parameter values.

\section{Discussion}

Results from our experiments were generally in accord with what we anticipated, but there were some surprises. The magnitude of the trends in the fishing mortality coefficient had no significant $(P<0.05)$ effect on the bias of the Synthesis estimates and relatively small influence on their variability. This seems to contradict the well known fact from Virtual Population Analysis that estimates of stock size converge more rapidly to their true values when there are high rates of fishing mortality (Pope 1972). Also, we found that bias and variability in the estimates of ending biomass, ending exploitable biomass, ending fishing mortality, and ending recruitment were larger when the fishery selectivity curve was asymptotic rather than domed. In contrast, Bence et al. (1993) in a Monte Carlo investigation of the Stock Synthesis program found that biomass estimates were more accurate if derived using data from a survey that had an asymptotic rather than domed selectivity curve. In their simulated populations the fishery selection curve was always domed. In our study the survey selection curve was always asymptotic.

The study reported here is only the first in a series of experiments. We intend to conduct additional experiments that will examine the influence of other forms of input data error including random variation in discards and variability in estimates of average weight at age. Also, there usually is 


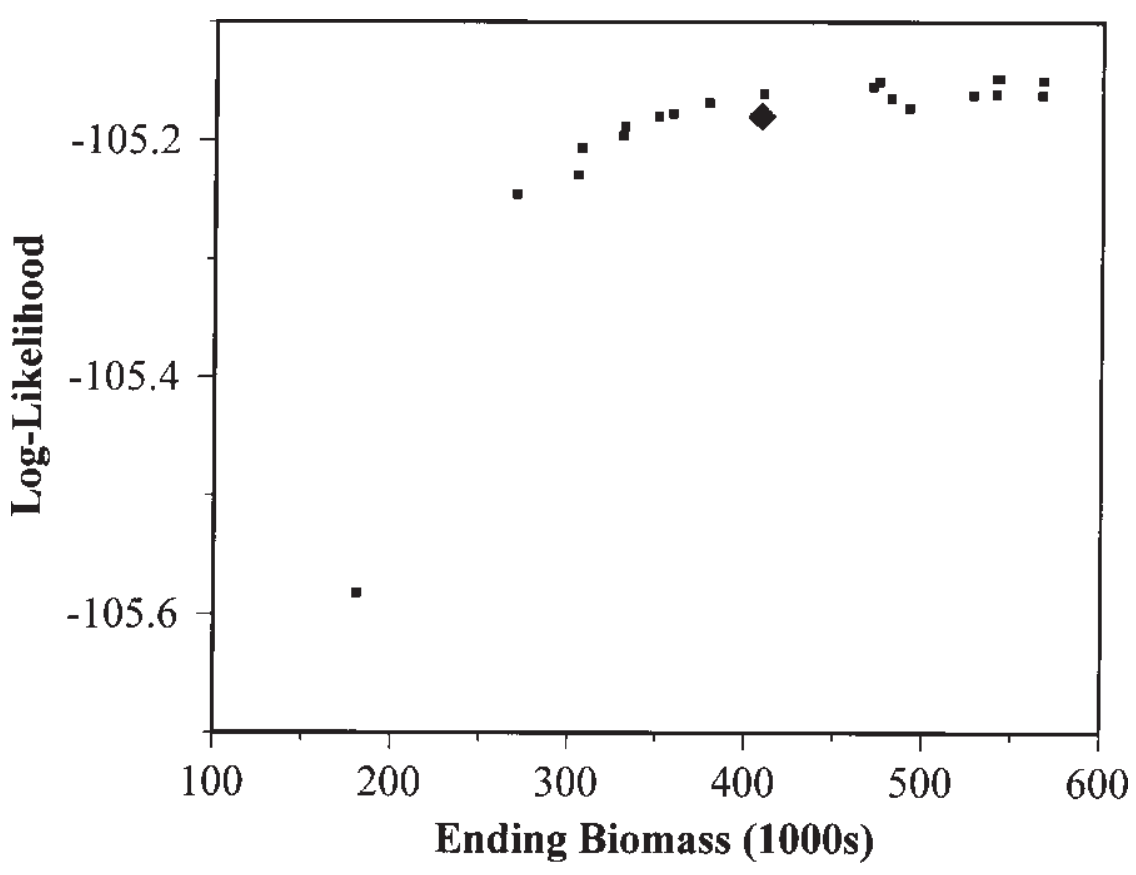

Figure 2. Stock Synthesis estimates can be sensitive to the choice of initial parameter values. Twenty sets of randomized initial parameter values here produced highly variable ( $C V=27 \%)$ estimates of ending biomass (shown as small squares) when applied to one particular random data set generated using experimental treatment 6 . Several other random data sets produced similar, but less extreme, patterns. In the main experiment the Stock Synthesis program always started with the true parameter values. The large diamond shows the biomass and likelihood values obtained by starting with the true parameter values. The true ending biomass for this experimental treament was 54,500. 
considerable sample to sample variation in age composition data (Crone 1995, Smith and Maguire 1983) and it is unrealistic to treat age composition data as simple multinomial random variables. We intend to explore the influence on Stock Synthesis estimates of a compound multinomial error structure for age composition data and evaluate the suggestion (Fournier and Archibald 1982) that age sample sizes in the likelihood specification be limited to 400 fish per sample.

Besides data errors, there are other potentially important sources of uncertainty in assessments based on the Stock Synthesis program. For example, structural errors, such as an incorrect assumption that fishery selectivity has been constant from year to year, can produce seriously inaccurate estimates of stock size (Sampson 1993). Other important structural errors that we want to investigate include how measurement errors are specified (e.g., normal versus lognormal) and whether or not mortality effects and recruitment include process error in addition to measurement error.

Stock Synthesis estimates of ending exploitable biomass form the basis for the annual catch quotas for many groundfish stocks on the U.S. Pacific coast (PFMC 1996). With respect to variability in the input data, the results of this study suggest that Synthesis estimates of exploitable biomass are only slightly biased and are relatively less variable than the input data. The ANOVA model for ending exploitable biomass predicts that the most variable estimates of ending exploitable biomass will occur for a treatment with asymptotic fishery selectivity, a low natural mortality coefficient $(0.2 / \mathrm{yr})$, variable recruitment, a low trend in the fishing mortality coefficient $(0.01 / \mathrm{yr})$, a short data series (8 years), small fishery and survey age composition samples (100 fish per annual sample), and high variability (80\%) in the fishing effort and survey biomass indices. For the particular set of parameter values that we examined for this worst-case scenario the ANOVA model predicts a $70.0 \%$ coefficient of variation in the estimate of ending exploitable biomass, and it predicts that a four-fold increase in the size of the annual age composition samples (to 400 fish per sample) would reduce the relative variability to $48.5 \%$. In contrast, a four-fold decrease in the relative variability of the survey biomass index would only reduce the relative variability to $58.7 \%$. This implies that increased port sampling to obtain more age composition data would produce greater improvements in assessment precision than increased research surveys to obtain more precise biomass indices. Furthermore, it would very likely be less expensive to collect the additional age composition data. For example, a four-fold improvement in the precision of a survey biomass estimate would require roughly a sixteen-fold increase in sampling because, under simple random sampling, the standard error of a biomass estimate is inversely proportional to the square root of the number of samples. 


\section{Acknowledgments}

This research was partially supported by grant no. NA36RG0451 (Project no. R/OPF-50) from the National Oceanic and Atmospheric Administration to the Oregon Sea Grant College Program and by appropriations made by the Oregon State legislature. The views expressed herein are those of the authors and do not necessarily reflect the views of NOAA or any of its subagencies.

\section{References}

Bence, J.R., A. Gordoa, and J.E. Hightower. 1993. Influence of age-selective surveys on the reliability of stock synthesis assessments. Can. J. Fish. Aquat. Sci. 50:827840.

Box, G.E.P., W.G. Hunter, and J.S. Hunter. 1978. Statistics for experimenters: An introduction to design, data analysis, and model building. Wiley and Sons, New York. 653 pp.

Crone, P.R. 1995. Sampling design and statistical considerations for the commercial groundfish fishery of Oregon. Can. J. Fish. Aquat. Sci. 52:716-732.

Deriso, R.B., T.J. Quinn II, and P.R. Neal. 1985. Catch-age analysis with auxiliary information. Can. J. Fish. Aquat. Sci. 42:815-824.

Fournier, D., and C.P. Archibald. 1982. A general theory for analyzing catch at age data. Can. J. Fish. Aquat. Sci. 39:1195-1207.

Gavaris, S., and C.A. Gavaris. 1983. Estimation of catch at age and its variance for groundfish stocks in the Newfoundland region. Can. Spec. Publ. Fish. Aquat. Sci. 66:178-182.

Kimura, D.K. 1989. Variability in estimating catch-in-numbers-at-age and its impact on cohort analysis. Can. Spec. Publ. Fish. Aquat. Sci. 108:57-66.

Megrey, B.A. 1989. Review and comparison of age-structured stock assessment models from theoretical and applied points of view. Amer. Fish. Soc. Symp. 6:8-48.

Methot, R.D. 1990. Synthesis model: An adaptable framework for analysis of diverse stock assessment data. Int. North Pac. Fish. Comm. Bull. 50:259-277.

Mohn, R.K. 1983. Effects of error in catch and effort data on tuning Cohort Analysis, with a postscript on logistic production models. Can. Spec. Publ. Fish. Aquat. Sci. 66:141-150.

Pacific Fishery Management Council. 1996. Status of the Pacific Coast groundfish fishery through 1996 and recommended acceptable biological catches for 1997. Pacific Fishery Management Council, Portland, OR.

Pelletier, D., and P. Gros. 1991. Assessing the impact of sampling error on modelbased management advice: Comparison of equilibrium yield per recruit variance estimators. Can. J. Fish. Aquat. Sci. 48:2129-2139. 
Pope, J.G. 1972. An investigation of the accuracy of Virtual Population Analysis using cohort analysis. Int. Comm. Northwest Atl. Fish. Res. Bull. 9:65-74.

Pope, J.G., and D.J. Garrod. 1975. Sources of error in catch and effort quota regulations with particular reference to variations in the catchability coefficient. Int. Comm. Northwest Atl. Fish. Res. Bull. 11:17-30.

Pope, J.G., and J.G. Shepherd. 1982. A simple method for the consistent interpretation of catch-at-age data. J. Cons. Cons. Int. Explor. Mer 40:176-184.

Prager, M.H., and A. MacCall. 1988. Sensitivities and variances of Virtual Population Analysis as applied to the mackerel, Scomber japonicus. Can. J. Fish. Aquat. Sci. 45:539-547.

Quinn II, T.J., E.A. Best, L. Bijsterveld, and I.R. McGregor. 1983. Sampling Pacific halibut Hippoglossus stenolepis landings for age-composition: History, evaluation, and estimation. Int. Pac. Halibut Comm. Sci. Rep. 68. 56 pp.

Restrepo, V.R., J.M. Hoenig, J.E. Powers, J.W. Baird, and S.C. Turner. 1992. A simple simulation approach to risk and cost analysis, with applications to swordfish and cod fisheries. Fish. Bull. U.S. 90:736-748.

Rivard, D. 1983. Effects of systematic, analytical, and sampling errors on catch estimates: A sensitivity analysis. Can. Spec. Publ. Fish. Aquat. Sci. 66:114-129.

Rivard, D. 1989. Overview of the systematic, structural, and sampling errors in cohort analysis. Amer. Fish. Soc. Symp. 6:49-65.

Rubinstein, R.Y. 1981. Simulation and the Monte Carlo Method. John Wiley and Sons, New York. 278 pp.

Sampson, D.B. 1987. Variance estimators for Virtual Population Analysis. J. Cons. Cons. Int. Explor. Mer 43:149-158.

Sampson, D.B. 1993. The assumption of constant selectivity and the stock assessment for widow rockfish. Fish. Bull. U.S. 91:676-689.

Shepherd, J.G., and M.D. Nicholson. 1991. Multiplicative modelling of catch-at-age data, and its application to catch forecasts. J. Cons. Cons. Int. Explor. Mer 47:284-294.

Smith, S.J., and J.J. Maguire. 1983. Estimating the variance of length composition samples. Can. Spec. Publ. Fish. Aquat. Sci. 66:165-170. 


\title{
Fuzzy Regression in Fisheries Science: Some Methods and Applications
}

\author{
Saul B. Saila \\ University of Rhode Island, Narragansett, Rhode Island \\ Scott Ferson \\ Applied Biomathematics, Setauket, New York
}

\begin{abstract}
Methodology for fuzzy regression is described and then illustrated with some applications to published data from the fisheries literature. Fuzzy regression should be a useful alternative or complement to conventional statistical regression whenever the relationship between variables is imprecise, data are imprecise, or sample sizes are very small. Formulations of fuzzy regression models are described, and goodness-of-fit criteria are briefly investigated and compared. The fuzzy regression technique is first illustrated by applications to data from the relationship between the proportion of chinook salmon jacks entering a river and the proportion of jacks in the ensuing cohort. A second example showing the relation between chinook salmon yearlings and water flow is also investigated. The third example deals with the relationship between an abundance index and virtual population analysis results for a short-time series of Atlantic mackerel data. Fuzzy regression methodology seems relatively straightforward, but it usually involves a constrained minimization problem, which may require mathematical programming methods. On the basis of this review and applications, we believe that fuzzy regression has utility in some fisheries-related applications, but further evaluation is suggested.
\end{abstract}

\section{Introduction}

Regression methods have been widely used and critiqued for some time in fishery science related applications, as is evident from a review by Ricker (1973). Sparre and Venema (1992) describe 18 equations from methods used in fishery science based on transformation and ordinary least square 
regression models of the form: $y=a+b x$. Prairie (1996) indicated that nearly $45 \%$ of the articles in the 1990 volume of the Canadian Journal of Fisheries and Aquatic Sciences contained at least one regression analysis. Clearly, regression models are ubiquitous in fishery science today. Ryan (1997) provides an excellent description of recent developments, as well as background in regression methodologies.

In general, a necessary characteristic of problems suitable for statistical regression analysis is that sufficient data should be available for drawing a valid statistical relation between the dependent and independent variable. Another important characteristic of a linear regression model in practical applications is the appropriateness of the statistical model, including the linearity assumption about the underlying functional relationship but also the assumptions of error-free $x$-values, homescedasticity, and independence. Fuzzy linear regression was first described by Tanaka et al. (1982), and it provides a means for addressing problems which fail to satisfy one or both of these characteristics. Relationships based on relatively few experimental data are common in the fisheries literature. For example, sparse data and an imprecise relationship between variables are illustrated by Fig. 1, redrawn from Cada et al. (1997, Fig. 2, page 136). Unwin and Glova (1997) contained several similar Fig.s. In many instances, scientists have used the relationship based on experience, judgment, or theoretical grounds, in spite of the fact that the statistical regression analysis alone may not lead to a strong conclusion.

Statistical regression analysis provides, under certain assumptions, a means to analyze the dependence among variables using statistical properties of the relationship. Consider a linear regression with one independent variable $x$ and one dependent variable $y$ for which the assumed form $y=a+b x$ represents a straight line. Given a set of observed data $\left(x_{1}, y_{1}\right)$, $\left(x_{2}, y_{2}\right), \ldots,\left(x_{n}, y_{n}\right)$ for the pair of variables $(x, y)$, familiar ordinary least squares (OLS) techniques are used to find values of $a$ and $b$ for which the total error of the estimated points on the straight line with respect to the observed points is minimized.

In the case of large data sets, statistical techniques can be effectively used to assess measurement or modeling error. However, in many practical fisheries applications, it often occurs that there are so few data available that statistical regression analysis cannot or should not be used. In such cases, the fishery scientist is faced with two alternatives. One is to disregard the relationship even when it is believed that it really exists. For example, most fishery scientists would agree that Pacific salmon smolt survival and travel time to the sea are related in spite of sparse data and ambiguous evidence about the relation between variables. The other alternative is to construct a regression line and calculate indices, such as the coefficient of determination $\left(r^{2}\right)$ which roughly evaluates the fit, or to show confidence intervals. This latter approach was used in Fig. 1.

The techniques of fuzzy regression may provide another alternative which has not been explored in a fisheries context to date and which may 


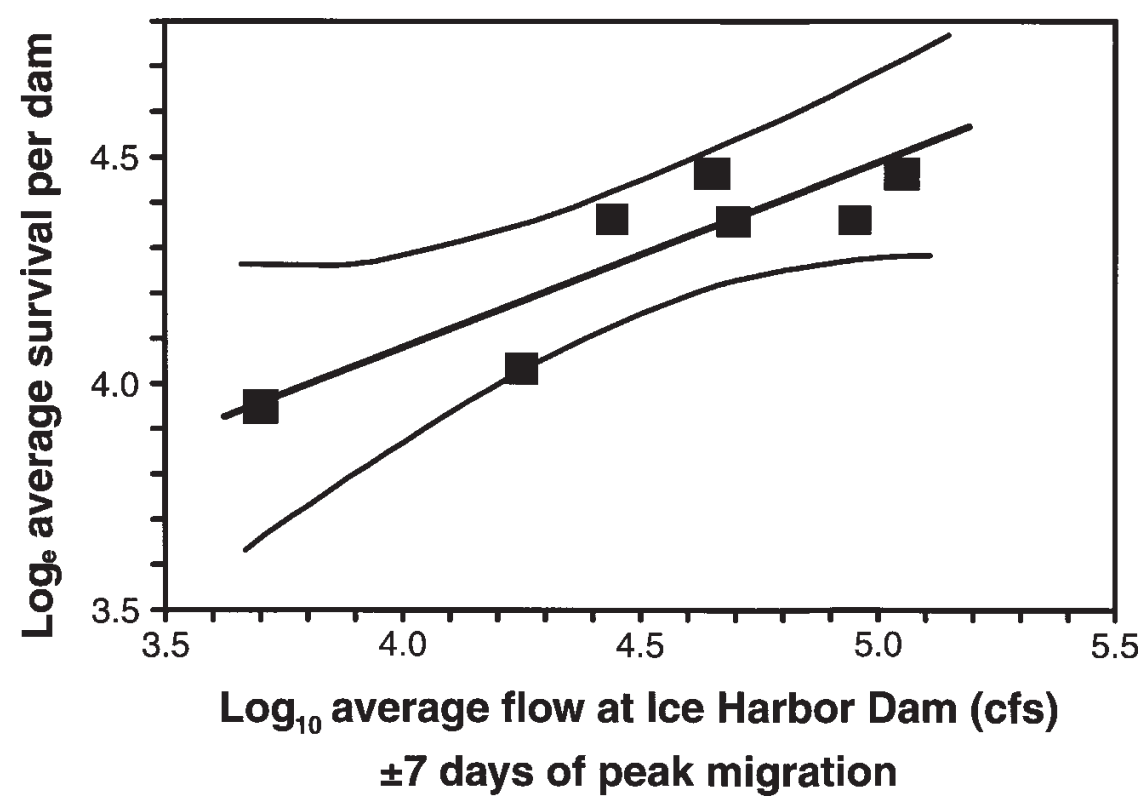

Figure 1. Ordinary least squares regression of river flow at Ice Harbor Dam versus yearling chinook salmon survival. Redrawn from Cada et al. (1997).

contribute to regression problems lacking an abundance of data or having a vague relationship between the variables. Briefly, fuzzy regression is a method of calibrating a fuzzy numerical relationship embodied in an equation involving fuzzy numbers (Kaufmann and Gupta 1985). The regression parameters may be fuzzy numbers and so describe the degree of acceptance of values for a parameter. As shown by Bárdossy et al. (1990), if the regression parameters are fuzzy numbers, the dependent variable is also a fuzzy number. The objective of fuzzy regression is to minimize some measure of "vagueness" of the dependent variable. Bárdossy et al. (1990) provided a good introduction to fuzzy regression in the context of hydrology using an example describing the relationship between soil electrical resistance and hydraulic permeability. Our intention is to provide a similar introduction to fishery scientists using fishery-related examples.

Johnson and Ayyub (1996) used fuzzy regression in a civil engineering application to model uncertainty in the prediction of bridge pier scour. They illustrate the use of fuzzy factors describing the bias between observed field data and estimates derived from laboratory or preliminary field data. This bias exists because of the use of small-scale experiments to model large-scale and real-world problems. The use of small-scale experiments to describe large-scale phenomena is also a problem in fisheries applications such as projecting the results of small-scale net behavior 
from flume studies to real-world trawl behavior. The above-mentioned study may be helpful in resolving similar problems in fishing gear technology research.

In summary, there seem to be at least two major motivations for applying fuzzy regression. The first one results from questions concerning the validity of a linear model for the given variables. The second motivation results from the nature of the data, which in some applications are inherently vague, very sparse, or both. These two motivations lead to two types of fuzzy regression analyses. One involves conventional crisp data and fuzzy parameters, and the other involves crisp parameters and fuzzy data, where the term "crisp" denotes ordinary, single values from the real number line. Fuzzy numbers possess a natural capability to express observation and measurement uncertainties that traditional crisp numbers do not have. In the material below, we will employ the approach of Klir and Yuan (1995). The next section describes an analysis under the first motivation-namely, the validity of the regression form used.

\section{Linear Regression with Fuzzy Parameters (LRFP)}

In this case, the dependence of an output variable $Y$ on input variables is expressed as:

$$
Y=C_{1} x_{1}+C_{2} x_{2}+\ldots C_{m} x_{m}
$$

where $C_{1}, C_{2}, \ldots, C_{m}$ are fuzzy numbers, and $x_{1}, x_{2}, \ldots, x_{m}$ are crisp (realvalued) input variables. For each $m$-tuple of input variable values, the output variable defined by $Y$ in equation (1) is a fuzzy number. Other definitions related to fuzzy regression are defined in Appendix A of Bárdossy et al. (1990). The objective of the regression analysis is to find fuzzy parameters $C_{1}, C_{2}, \ldots, C_{m}$ for which equation (1) provides the best agreement with the data, according to a goodness-of-fit criterion, such as minimizing the sum of the widths of the fuzzy regression coefficients.

Assume the parameters in equation (1) are symmetric triangular fuzzy numbers defined by

$$
C_{j}(c)= \begin{cases}1-\frac{\left|c-c_{j}\right|}{s_{j}} & \text { when } c_{j}-s_{j} \leq c \leq c_{j}+s_{j} \\ 0 & \text { otherwise }\end{cases}
$$

where $c_{j}$ is the point for which $C_{j}\left(c_{j}\right)=1$, and $s_{j}>0$ is the spread of $C_{j}$. Each $C_{j}$ can therefore be denoted by $\left(c_{j}, s_{j}\right)$ for $j=1,2, \ldots, m$. It can then be proved by the extension principle (Zadeh 1965) that $Y$ in equation (1) is also a symmetric fuzzy number, which is given by 


$$
Y(y)= \begin{cases}1-\frac{\left|y-\boldsymbol{x}^{\mathrm{T}} \boldsymbol{c}\right|}{\boldsymbol{s}^{\mathrm{T}}|\boldsymbol{x}|} & \text { when } \boldsymbol{x} \neq 0 \\ 1 & \text { when } \boldsymbol{x}=0, y \neq 0 \\ 0 & \text { when } \boldsymbol{x}=0, y=0\end{cases}
$$

for all $y \in \Re$, where $\mathrm{T}$ denotes the transposition and

$$
\boldsymbol{x}=\left(\begin{array}{c}
x_{1} \\
x_{2} \\
\cdot \\
\cdot \\
\cdot \\
x_{m}
\end{array}\right), \boldsymbol{c}=\left(\begin{array}{c}
c_{1} \\
c_{2} \\
\cdot \\
\cdot \\
\cdot \\
c_{m}
\end{array}\right), \boldsymbol{s}=\left(\begin{array}{c}
s_{1} \\
s_{2} \\
\cdot \\
\cdot \\
\cdot \\
s_{m}
\end{array}\right),|\boldsymbol{x}|=\left(\begin{array}{c}
\left|x_{1}\right| \\
\left|x_{2}\right| \\
\cdot \\
\cdot \\
\cdot \\
\left|x_{m}\right|
\end{array}\right)
$$

Finding the fuzzy parameters $C_{1}, C_{2}, \ldots, C_{m}$ can be converted to the problem of finding the vectors $\mathbf{c}$ and $\mathbf{s}$ such that the $Y(y)$ functions given by equation (3) fit the data as well as possible. Each data point $\left(x_{i}, y_{i}\right)$ consists of a vector of observed values of the independent variables $x_{i}$, and the corresponding observed value of the dependent variable $y_{i}$. Let $Y_{i}(y)$ denote the fuzzy number defined by equation (3) for the $i$ th vector of independent variables. The fuzzy parameters are fitted so that they are as narrow as possible while permitting the values of $Y_{i}(y)$ to be large for the values $y_{i}$ represented in the observed data. In particular, for each vector of independent variables $x_{i}$, the associated $y_{i}$ should belong to the corresponding fuzzy number $Y_{i}$ with a grade that is greater than or equal to some given value $h \in[0,1]$. That is, $Y_{i}\left(y_{i}\right) \geq h$ for each $i=1,2, \ldots, n$. The linear regression with fuzzy parameters problem can be expressed in terms of a classical linear programming problem illustrated by Klir and Yuan (1995, page 456) as

$$
\text { Minimize } \sum_{j=1}^{m} s_{j} \text {, }
$$

Subject to

$$
\begin{gathered}
(1-h) \mathbf{s}^{\mathrm{T}}\left|\mathbf{x}_{i}\right|-\left|y_{i}-\mathbf{x}_{i}^{\mathrm{T}} \mathbf{c}\right| \geq 0, \quad i=1,2, \ldots, n \\
\mathrm{~s}_{\mathrm{j}} \geq 0, \mathrm{j}=1,2, \ldots, \mathrm{m} .
\end{gathered}
$$


The minimization tightens the spread of the fuzzy parameters so that the model is as specific as possible. Without this minimization, it would always be possible to get $Y(y)$ to be larger than $h$ for any value of the dependent variable, simply by widening the spread of the fuzzy parameters. The positivity constraint insures that the fitted parameters are feasible fuzzy numbers.

An example of linear regression with fuzzy parameters is provided using the data in Table 1, which we extracted from Unwin and Glova (1997, Fig. 3). We assume the form $Y=C X$, where $C=(c, s)$ is a fuzzy parameter expressed by a symmetric fuzzy number. This linear programming problem has the form

$$
\begin{gathered}
\text { Minimize } s, \\
\text { Subject to } \\
8(1-h) s-|24-8 c| \geq 0, \\
9(1-h) s-|8-9 c| \geq 0, \\
\cdot \\
\cdot \\
47(1-h) s-|23-47 c| \geq 0, \\
s \geq 0 \text {, and } \\
h \in[0,1] \text { is a fixed number. }
\end{gathered}
$$

Some justification for the use of this form is provided by comparing of the results from fitting an ordinary least squares regression with intercept versus a regression through the origin. It was evident that an improvement in the $r^{2}$ value occurred for the latter case. Of course, as Ryan (1997) notes, $r^{2}$ values for these two forms of linear regression are not strictly comparable, and, in any case, Prairie (1996) pointed out that $r^{2}$ does not necessarily provide an intuitive measure of the predictive power of regressions. Nevertheless, the difference was large, and it also seemed intuitively evident that $Y=0$ when $x=0$, since no returns are expected if there are no spawners.

We used the optimization modeling software LINGO $^{\mathrm{TM}}$, which includes simultaneous linear and nonlinear solver-optimizers, to make the computations for this example. The mathematical programming problem is linear in this case. For the data from the Table 1 and the grade of membership set at 0.5 , we found $C=(0.872,1.32)$.

Fuzzy numbers $Y=C x$ can be calculated for various values of $x$. In this case, $C$ is a fuzzy parameter expressed by a symmetric triangular fuzzy 
Table 1. Raw data on chinook salmon spawners versus returns extracted from Unwin and Glova (1997) with predictions based on ordinary least squares regression (OLS), linear regression with fuzzy parameters (LRFP), and linear regression with fuzzy data (LRFD).

\begin{tabular}{|c|c|c|c|c|c|c|c|c|}
\hline & \multicolumn{2}{|c|}{ Raw data } & \multicolumn{2}{|c|}{ OLS } & \multicolumn{2}{|c|}{ LRFP } & \multicolumn{2}{|c|}{ LRFD } \\
\hline Case $^{\bar{S}}$ & $\begin{array}{c}\text { Spawners } \\
X\end{array}$ & $\begin{array}{c}\text { Returns } \\
Y\end{array}$ & $\begin{array}{c}\text { Predicted } \\
\text { returns }\end{array}$ & Residuals & $\begin{array}{l}\text { Predicted } \\
\text { returns }\end{array}$ & Residuals & $\begin{array}{l}\text { Predicted } \\
\text { returns }\end{array}$ & Residuals \\
\hline 1 & 8 & 24 & 7.03 & 16.97 & 6.98 & 17.02 & 6.91 & 17.09 \\
\hline 2 & 9 & 8 & 7.90 & 0.10 & 7.85 & 0.15 & 7.78 & 0.22 \\
\hline 3 & 11 & 15 & 9.66 & 5.34 & 9.59 & 5.41 & 9.50 & 5.50 \\
\hline 4 & 11 & 16 & 9.66 & 6.34 & 9.59 & 6.41 & 9.50 & 6.50 \\
\hline 5 & 15 & 18 & 13.17 & 4.83 & 13.02 & 4.92 & 12.96 & 5.04 \\
\hline 6 & 22 & 19 & 19.32 & -0.32 & 19.18 & -0.18 & 19.01 & -0.01 \\
\hline 7 & 25 & 33 & 21.96 & 11.04 & 21.80 & 11.20 & 23.60 & 5.40 \\
\hline 8 & 26 & 32 & 22.84 & 9.17 & 22.67 & 9.33 & 22.46 & 9.54 \\
\hline 9 & 30 & 26 & 26.35 & -0.35 & 26.16 & -0.16 & 25.96 & 0.08 \\
\hline 10 & 33 & 24 & 28.98 & -4.98 & 26.78 & -4.78 & 28.51 & -4.51 \\
\hline 11 & 29 & 52 & 25.47 & 26.53 & 25.29 & 26.71 & 25.51 & 26.94 \\
\hline 12 & 39 & 28 & 34.25 & -6.25 & 34.01 & -6.01 & 33.70 & -5.70 \\
\hline 13 & 44 & 31 & 38.64 & -7.64 & 38.37 & -7.37 & 38.02 & -7.02 \\
\hline 14 & 47 & 23 & 41.28 & -18.28 & 40.98 & -17.98 & 40.60 & -17.60 \\
\hline
\end{tabular}

For the fuzzy methods, the tabled values for predicted returns are the values where the fuzzy numbers produced by the regression equations have their peaks. This is analogous to using the means of the predictions from OLS (which are also distributions). Also shown are the residuals for each analysis.

number. Although mathematical operations for fuzzy arithmetic are relatively straightforward, they can become tedious. Risk Calc (Ferson et al. 1998) is a convenient environment for evaluating fuzzy mathematical expressions. These can include scalars representing known or mathematically defined integers and real numbers, intervals representing uncertain numbers about which bounds can be established, and fuzzy numbers representing uncertain numbers for which, in addition to having a range of possible values, one can say that some are more plausible than others. All these kinds of numbers can be mixed and used together in mathematical expressions. In our case, a real-valued independent variable is multiplied by a symmetric triangular fuzzy number (the slope of the regression) to obtain fuzzy predicted values of the dependent variable. The results are summarized numerically in Table 1 and also illustrated in Fig. 2 . The figure depicts the original data points, the regression line and, in their own graph, the predicted values $Y_{i}=C x_{i}$. Each of these predictions is a symmetric triangular fuzzy number whose peak is located at the best estimate and whose breadth indicates the reliability of the estimate. 


\section{Linear Regression with Fuzzy Data (LRFD)}

In this type of regression, the dependence of an output variable $Y$ on input variables $X_{i}$ is expressed by the following:

$$
Y=a_{1} X_{1}+a_{2} X_{2}+\ldots+a_{m} X_{m}
$$

where the values of the input and output variables are fuzzy numbers which are assumed to be triangular and symmetric, and $a_{1}, a_{2}, \ldots, a_{m}$ are real-valued parameters. Let $X_{j}=\left(x_{j}, s_{j}\right)$ for $j=1,2, \ldots, m$. Then

$$
Y(y)= \begin{cases}1-\frac{\left|y-\mathbf{a}^{\mathrm{T}} \mathbf{x}\right|}{\mathbf{s}^{\mathrm{T}}|\mathbf{a}|} & \text { when } \mathbf{a} \neq 0 \\ 1 & \text { when } \mathbf{a}=0, y \neq 0 \\ 0 & \text { when } \mathbf{a}=0, y=0\end{cases}
$$

for all $y \in \Re$, where

$$
\boldsymbol{a}=\left(\begin{array}{c}
a_{1} \\
a_{2} \\
\cdot \\
\cdot \\
\cdot \\
a_{m}
\end{array}\right), \boldsymbol{x}=\left(\begin{array}{c}
x_{1} \\
x_{2} \\
\cdot \\
\cdot \\
\cdot \\
x_{m}
\end{array}\right), \boldsymbol{s}=\left(\begin{array}{c}
s_{1} \\
s_{2} \\
\cdot \\
\cdot \\
\cdot \\
s_{m}
\end{array}\right) .
$$

Data are provided as pairs $\left(X_{i}, Y_{i}\right)$ for $i=1,2, \ldots, n$, where each $X_{i}$ is an $m$ tuple of symmetric triangular fuzzy numbers, and $Y_{i}$ is a symmetric triangular fuzzy number. The pair of real numbers $\left(x_{i j}, s_{i j}\right)$ will specify the fuzzy number for the $j$ th independent variable of the $i$ th data point. The pair of real numbers $\left(y_{i}, s_{i}\right)$ will specify the fuzzy number for the corresponding dependent variable for the $i$ th data point. Let $Y_{i}$ denote the observed fuzzy number associated with $X_{i}$, and let $\hat{Y}_{i}$ denote the predicted fuzzy number obtained from fuzzy linear function (5) when $X_{i}$ is used as its input. The objective of the regression problem is to find parameters $a_{1}, a_{2}, \ldots, a_{m}$ such that the fit between the fuzzy prediction $\hat{Y}_{i}$ and the fuzzy data $Y_{i}$ is as good as possible. According to Klir and Yuan (1995), two simultaneous criteria are usually used for goodness of fit. First, minimize the total differences between the areas of the fuzzy numbers $\hat{Y}_{i}$ and $Y_{j}$ for $i=1, \ldots, n$. Second, make the fuzzy numbers $\hat{Y}_{i}$ and $Y_{j}$ compatible to some given de- 


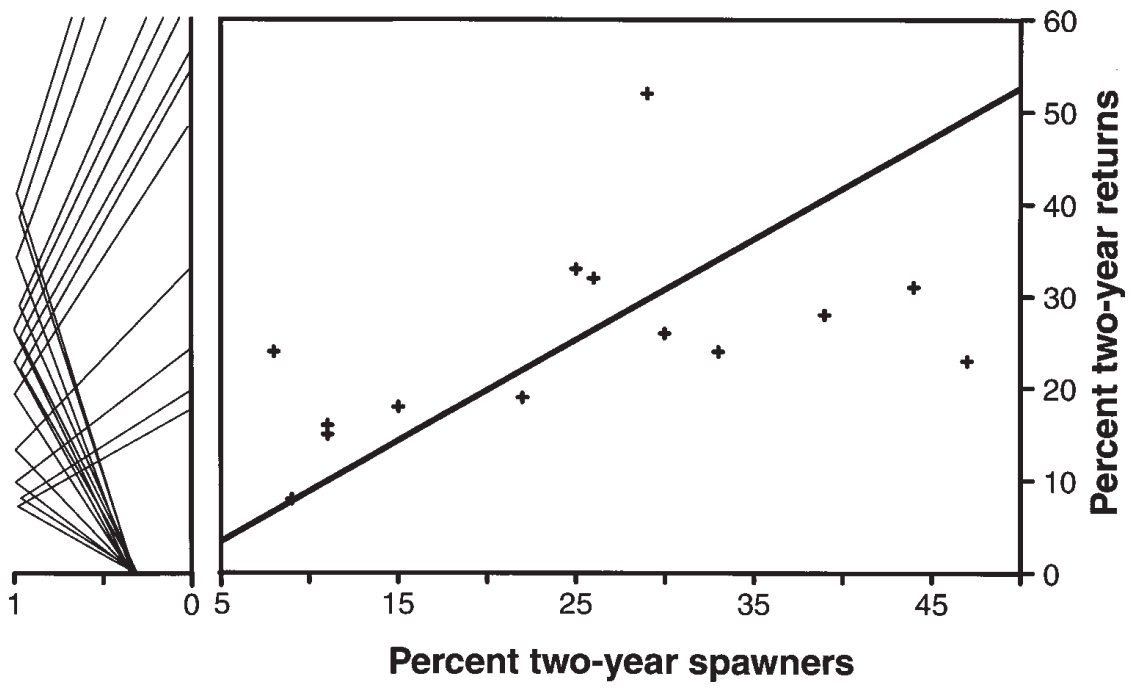

Figure 2. Linear regression with fuzzy parameters for the relationship between chinook salmon spawners and returns. The regression line is depicted in the graph on the right along with the original data from Unwin and Glova (1997) depicted as crosses. The small graph on the left is a rotated display of the fuzzy numbers predicted for returns by the regression for each $x$-value observed in the original data.

gree $h \in[0,1]$. This can be formulated as the optimization problem (Klir and Yuan 1995, page 456)

$$
\text { Minimize } \sum_{i=1}^{n}\left|s_{i}-\sum_{j=1}^{m}\right| a_{j}\left|s_{i j}\right| \text {, }
$$

Subject to

$$
\begin{gathered}
-\sum_{j=1}^{m}\left|a_{j}\right| s_{i j}+\sum_{j=1}^{m} a_{j} x_{i j} \leq y_{i}-s_{i}, \text { for all } i=1, \ldots, n, \\
\sum_{j=1}^{m}\left|a_{j}\right| s_{i j}+\sum_{j=1}^{m} a_{j} x_{i j} \geq y_{i}-s_{i}, \text { for all } i=1, \ldots, n,
\end{gathered}
$$

where $a_{i} \in \Re$ for all $j=1, \ldots, m$.

To illustrate the application of linear regression with fuzzy data, we reanalyzed a subset of the data used in the previous example. For this example, however, the independent and dependent variables are now presumed to be fuzzy numbers which characterize the measurement error or 


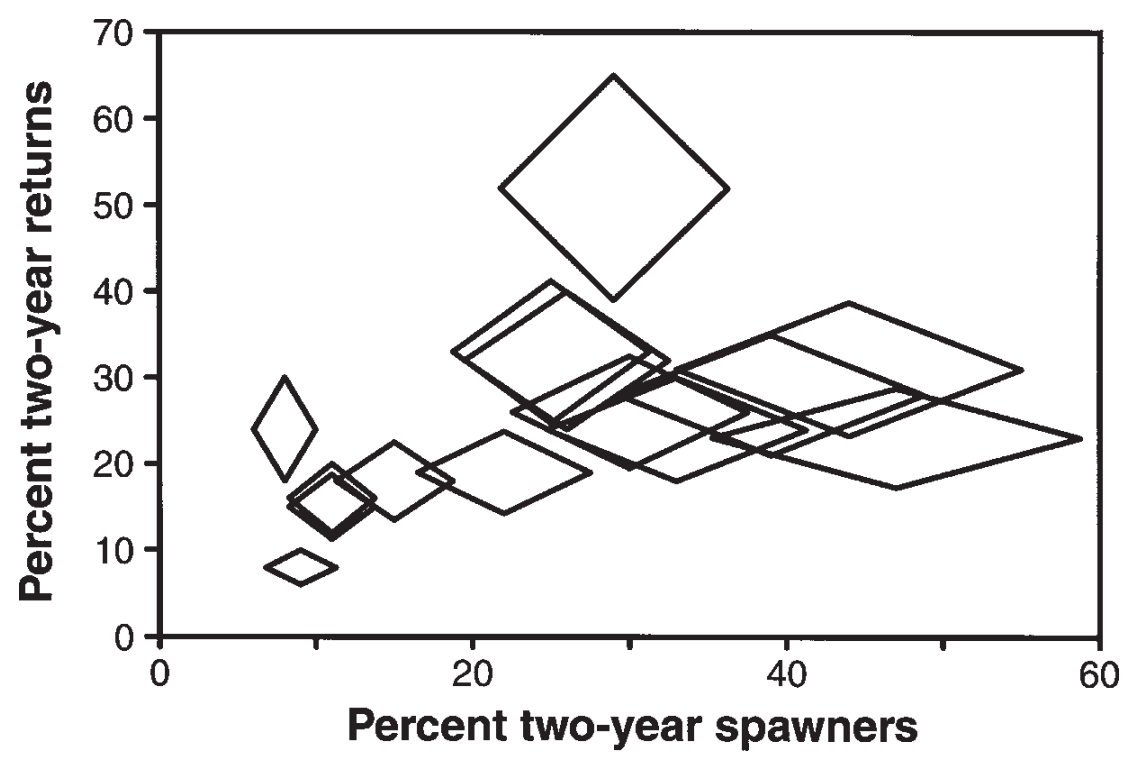

Figure 3. Data to be used in a linear regression with fuzzy data. Based on the chinook salmon data of Unwin and Glover (1997). Each diamond represents a pair of fuzzy numbers.

ambiguity of the values. Although the regression technique can accept fuzzy numbers with arbitrary uncertainties, for the sake of simplicity, we assumed the uncertainties to be proportional to the estimated magnitude. The data, which are displayed in Fig. 3, are therefore bivariate symmetric triangular fuzzy numbers, each with a breadth in each dimension of $\pm 25 \%$ the original value. The LINGO optimization modeling language yielded the solution (according to the chosen criteria) $Y=0.864 X$.

Tables 1 and 2 summarize and compare the results from three kinds of linear regression on the Unwin and Glova data set. Table 1 gives the (central) predicted values and the residuals for ordinary least squares regression (OLS), linear regression with fuzzy parameters (LRFP), and linear regression with fuzzy data (LRFD). Predicted values are presented for each $x$-value observed in the data set. Residuals are the differences between the observed and predicted values for the $Y$ variable. Values are displayed to two decimal places for the benefit of those who attempt to repeat these calculations, although this may not reflect the precision of the data set.

Table 2 compares the three regressions using quality coefficients defined by Pop and Sarbu (1996). QC1 is a coefficient used in analytical chemistry and elsewhere to judge the goodness of fit to a regression line. QC5 relativizes residuals by the maximum of absolute residuals and has a 
Table 2. Values of quality coefficients described by Pop and Sarbu (1996) for the three regressions on the chinook salmon data of Table 1 .

\begin{tabular}{|c|c|c|c|}
\hline \multirow[b]{2}{*}{ Coefficient typ } & \multicolumn{3}{|c|}{ Model type } \\
\hline & $\overline{\text { OLS }}$ & LRFP & $\overline{\text { LRFD }}$ \\
\hline$Q C 1$ & 0.81 & 0.82 & 0.82 \\
\hline$Q C 5$ & 1.6 & 1.6 & 1.5 \\
\hline NQC 5 & 0.21 & 0.21 & 0.18 \\
\hline QC6 & 5.0 & 5.0 & 5.1 \\
\hline NQC6 & 0.12 & 0.12 & 0.13 \\
\hline$Q C 1=$ & $\left.\frac{1}{n-1} \sum_{i=1}^{n}\left(\frac{r_{i}}{\hat{y}_{i}}\right)^{2}\right]^{1 / 2}$ & & \\
\hline$Q C 5=$ & $\sum_{i=1}^{n}\left(\frac{r_{i}}{\max \left|r_{i}\right|}\right)$ & NQC5 & \\
\hline$Q C 6=$ & {$\left[\sum_{i=1}^{n}\left(\frac{r_{i}}{r}\right)^{2}\right]^{1 / 2}$} & NQC6 & $\frac{\sqrt{n}}{n}$ \\
\hline
\end{tabular}

In the formulas for the coefficients given at the bottom of the table, $n$ is the number of data points, the $r_{i}$ are the residuals $\left(y_{i}-\hat{y}_{i}\right)$, where $y_{i}$ and $\hat{y}_{i}$ are observed dependent values at each datum and those predicted by the model respectively, and $r$ is the mean of the $r_{i}$.

range $\left[1, n^{1 / 2}\right]$. QC6 uses the mean of absolute residuals and has a range $\left[n^{1 / 2}, n\right]$. These coefficients were normalized by Pop and Sarbu (1996) as $N Q C 5$ and NQC 6 which take values between $[0,1]$ as more practical ranges for evaluative purposes. Table 2 shows that both fuzzy regressions compare reasonably well with the traditional least squares regression.

\section{Reexamination of Figure 1 Data}

The seven data points of Fig. 1 were analyzed by fitting a fuzzy regression (FR) with intercept to the data because it is the most commonly used form of regression. The fuzzy regression procedure used for this example was developed from an algorithm provided by Pop and Sarbu (1996) termed the Modified Fuzzy 1-Lines. Results obtained by using this algorithm were compared by the above authors with ordinary least squares and weighted least squares, as well as several robust regression methods. This fuzzy regression procedure was found to exceed the least squares method and equal or exceed all other methods tested including two previously pro- 
posed fuzzy methods. The criteria for comparison included the maximum of absolute residuals and the mean of absolute residuals as well as other preference measures. The Pop and Sarbu algorithm was converted into a FORTRAN 77 program and compared with the results from examples provided by the above-mentioned authors. Our results were virtually identical to those reported. We also implemented the quality coefficients used by Pop and Sarbu to evaluate the goodness of fit of the regression lines. The software is available from the authors.

The fuzzy regression program (FR) developed from the Modified Fuzzy 1-Lines algorithm produces the fuzzy number associated with the classical set $X$ to the membership threshold $\alpha(\alpha=0.05$ in our applications) together with its linear representation. Our program also estimates the five quality coefficients used by Pop and Sarbu, as well as a membership function describing the grade of membership for each observed point in the data set which may be useful in detecting outliers in the data.

Table 3 shows the results of comparing the OLS regression with intercept to the new fuzzy regression (FR) in terms of the five quality coefficients. Note that most of the quality coefficients using the fuzzy regression method were smaller than those derived from the OLS regression. This indicates that the performance of the fuzzy regression method was superior using these several quality coefficients.

\section{Calibration Program}

The problem addressed in this example is the so-called calibration problem which has received attention by statisticians as well as by scientists in many disciplines, including fishery scientists (ICES 1993). This problem can be loosely defined as a prediction from past data on the relationship between a precise and an imprecise measurement of the same thing, using a future imprecise observation to predict the more precise value. An example is the estimation of year class strength (recruitment) from a survey index, based on calibration against virtual population analysis (VPA) estimates of past year class strengths. We believe that the recent results from calibration studies in analytical chemistry using fuzzy regression (Pop and Sarbu 1996) may be useful in contributing to a better understanding of the calibration problem in a fisheries context.

We consider the estimation of year class strength from a survey index against VPA estimates of past year class strength for the northwest Atlantic mackerel. The data set is from Clark (1979), and it has also been used by the 1984 ICES Working Group on Methods of Fish Stock Assessments. We compare the results obtained by the Modified Fuzzy 1-Lines regression with results obtained by OLS regression with intercept based on logtransformed variables. The transformed data and the results obtained from the two regressions are shown in Table 4. From an examination of the residuals by year between the OLS and the FR methods, it is evident that the FR method had smaller residual values in five of the eight years. How- 
Table 3. Comparison of OLS versus FR regressions on the flow versus survival data from Cada et al. (1997) shown in Fig. 1.

\begin{tabular}{lll}
\hline Quality & \multicolumn{2}{c}{ Method } \\
\cline { 2 - 3 } coefficients & OLS & FR \\
\hline QC1 & 0.026 & 0.025 \\
QC5 & 1.9 & 1.4 \\
NQC5 & 0.55 & 0.25 \\
QC6 & 3.5 & 2.7 \\
NQC6 & 0.20 & 0.23 \\
\hline
\end{tabular}

ever, the quality coefficients indicate slightly higher (i.e., worse) values for the FR method. This is not altogether surprising. By design, the OLS regression yields the line of best fit as measured by the sum of squared residuals. The quality coefficients are closely related to this criterion. The FR method may be useful as another form of robust regression that is insensitive to large residuals and therefore appropriate for data sets which may contain outliers.

\section{Discussion and Conclusions}

Fuzzy regression has been described and illustrated with some simple examples from recent fisheries literature describing important relationships based on relatively limited data. The results suggest that fuzzy regression compares favorably with standard statistical regression procedures in terms of performance as measured by certain quality coefficients. We believe fuzzy regression can overcome some of the weaknesses of OLS. Justification for these statements is provided by the following observations.

In the case of applying linear regression with fuzzy parameters (LRFP) and linear regression with fuzzy data (LRFD) to the chinook salmon returns example (Table 1), the residuals from both types of fuzzy regression are very similar to the least squares residuals. The comparison of quality coefficients (Table 2) also indicates that the performances for all methods are quite similar.

The quality coefficients (Table 3 ) also have very similar values for traditional and fuzzy regressions of the flow versus survival relationship depicted in Fig. 1. Four out of the five coefficients, however, suggest the fuzzy regression is superior. 
Table 4. Raw data on northwest Atlantic mackerel survey indices versus VPA results based on Clark (1979) comparing fuzzy regression (FR) and ordinary least squares (OLS) regression for log-transformed data.

\begin{tabular}{|c|c|c|c|c|c|c|}
\hline \multirow[b]{2}{*}{ Case } & \multicolumn{2}{|c|}{ Transformed data } & \multicolumn{2}{|c|}{ OLS } & \multicolumn{2}{|c|}{ FR } \\
\hline & Index & $\begin{array}{c}\text { VPA } \\
\text { Recruits }\end{array}$ & $\begin{array}{c}\text { VPA } \\
\text { Predicted }\end{array}$ & Residuals & $\begin{array}{c}\text { VPA } \\
\text { Predicted }\end{array}$ & Residuals \\
\hline 1 & 0.000 & 6.68 & 6.85 & -0.17 & 6.90 & -0.05 \\
\hline 2 & 0.406 & 7.31 & 6.97 & 0.35 & 7.02 & 0.30 \\
\hline 3 & 1.610 & 7.35 & 7.31 & 0.04 & 7.35 & -0.00 \\
\hline 4 & 1.792 & 7.09 & 7.36 & -0.27 & 7.40 & -0.31 \\
\hline 5 & 1.946 & 7.17 & 7.40 & -0.23 & 7.44 & -0.27 \\
\hline 6 & 2.526 & 7.74 & 7.57 & 0.17 & 7.60 & 0.14 \\
\hline 7 & 2.603 & 7.70 & 7.59 & 0.10 & 7.63 & 0.07 \\
\hline \multirow[t]{7}{*}{8} & 3.091 & 7.74 & 7.73 & 0.01 & 7.76 & 0.02 \\
\hline & & \multicolumn{3}{|c|}{ Quality coefficients } & & \\
\hline & & $Q C 1$ & 0.030 & 0.030 & & \\
\hline & & QC5 & 1.6 & 1.9 & & \\
\hline & & $N Q C$ & 0.34 & 0.47 & & \\
\hline & & QC6 & 3.4 & 3.5 & & \\
\hline & & $N Q C$ & 0.10 & 0.12 & & \\
\hline
\end{tabular}

For the Atlantic mackerel data, the results are again very similar (Table 4), although the OLS results seem to be slightly but consistently higher. The FR intercept value for the fuzzy regression was 6.85 versus 6.90 for the least-squares regression. The slope of the fuzzy regression was 0.278 versus 0.288 for the OLS model. This suggests that the FR model weights large values of residuals less than the OLS model.

It should be recalled that OLS regression is based on the assumption of an independent and normal error distribution with uniform variance (homoscedasticity). In fisheries applications such as the calibration problem, the $y$-direction error is dependent on the survey index value and on the presence of outliers which lead to heteroscedastic results. In practice, we don't usually know the shape of the error distribution function and its variance. A consequence is that the least squares method does not lead to the maximum likelihood estimate. If the tails of the experimental error distribution contain a much larger percentage of the total area than the normal distribution, the best linear unbiased estimator may not be very good. The least squares method is well known to be sensitive to the effect 
of large residuals, so results are distorted if there are large differences between the observed data and the model predictions. Fuzzy regression appears to overcome some of these difficulties.

A basic issue relates to the interpretation of fuzzy regression results. These cannot be interpreted in the same way as the results from classical regression. The prediction from a fuzzy regression is a fuzzy number for each value of the independent variable(s). From a practical point of view, we believe that the results from fitting a fuzzy regression to uncertain or imprecise data may provide a fishery manager with a more conservative but realistic indication of the variability and uncertainty associated with a predicted response. This is in keeping with the so-called precautionary approach to fisheries management.

This work has only briefly introduced the use of fuzzy regression. It can be extended to multiple independent variables (e.g., Heshmaty and Kandel, 1985) and can also be applied to quadratic and other polynomial relationships (Bárdossy 1990). The results of this work can be briefly summarized as follows:

1. Fuzzy regression leads to a mathematical programming problem which can be solved in a straightforward manner.

2. Rules of fuzzy mathematics are used to define the regression parameters on fuzzy numbers.

3. Results of fuzzy regression applications indicate that it compares favorably with ordinary least squares regression.

4. Our limited experience in applying fuzzy regression to some fisheries-related data suggests that it may be useful in overcoming some difficulties associated with heteroscedastic and uncertain data.

\section{References}

Bárdossy, A. 1990. Note on fuzzy regression. Fuzzy Sets and Systems 37:65-75.

Bárdossy, A., I. Bogardi, and L. Duckstein. 1990. Fuzzy regression in hydrology. Water Resour. Res. 26(7):1497-1508.

Cada, G.F., M.D. Duncan, S.V. Mitz, and M.S. Bavelhimer. 1997. Effects of water velocity on the survival of downstream-migrating juvenile salmon and steelhead: A review with emphasis on the Columbia River Basin. Reviews in Fisheries Science 5(2):131-183.

Clark, S.N. 1979. Application of bottom-trawl survey data to fish stock assessment. Fisheries 4(3):9-15.

Ferson, S., W. Root, and R. Kuhn. 1998. RAMAS Risk Calc: Risk assessment with uncertain numbers. Applied Biomathematics, Setauket, NY.

Heshmaty, B., and A. Kandel. 1985. Fuzzy linear regression and its applications to forecasting in uncertain environments. Fuzzy Sets and Systems 15:159-191. 
ICES. 1993. Reports of the working group on methods of fish stock assessments. ICES Coop. Res. Rep. 191.

Johnson, P.A., and B.A. Ayyub. 1996. Modeling uncertainty in prediction of pier scour. Journal of Hydraulic Engineering, 1 February 1996:66-76.

Kaufmann, A., and M.M. Gupta. 1985. Introduction to fuzzy arithmetic: Theory and applications. Van Nostrand Reinhold, New York.

Klir, G.J., and B. Yuan. 1995. Fuzzy sets and fuzzy logic: Theory and applications. Prentice Hall, Upper Saddle River, NJ.

Pop, H.F., and C. Sarbu. 1996. A new fuzzy regression algorithm. Anal. Chem. 66:771778.

Prairie, Y.T. 1996. Evaluating the predictive power of regression models. Can. J. Fish. Aquat. Sci. 53:490-492.

Ricker, W.E. 1973. Linear regressions on fishery research. J. Fish. Res. Board Can. 30:409-434.

Ryan, T.R. 1997. Modern regression methods. J. Wiley and Sons, New York.

Sparre, P., and S.C. Venema. 1992. Introduction to tropical fish stock assessment. FAO Fish. Tech. Pap. 306/1, Rev. 1.

Tanaka, H., S. Uijima, and K. Asai. 1982. Linear regression analysis with fuzzy model. IEEE Trans. on Systems, Man, and Cybernetics 12(6):903-907.

Unwin, M.J., and G.J. Glova. 1997. Changes in life history parameters on a naturally spawning population of chinook salmon (Oncorhynchus tshawytscha) associated with release of hatchery-raised fish. Can. J. Fish. Aquat. Sci. 54:12351245.

Zadeh, L.A. 1965. Fuzzy sets. Information and Control 8(3):338-353. 


\title{
Evaluation of Assumed Error Structure in Stock Assessment Models That Use Sample Estimates of Age Composition
}

\author{
Paul R. Crone \\ National Marine Fisheries Service, Northwest Fisheries Science Center, \\ Hatfield Marine Science Center, Newport, Oregon \\ David B. Sampson \\ Oregon State University, Hatfield Marine Science Center, Newport, Oregon
}

\section{Abstract}

The sampling error associated with estimates of age composition for five groundfish species commercially landed at Oregon ports is used to examine the ability of age-structured stock assessment models to adequately describe the stochastic properties of actual catch-at-age data. Specifically, estimated coefficients of variation associated with samples of catch-atage are presented graphically to evaluate a theoretical consideration involved in stock assessment models widely used in marine fishery management. Results presented here indicate that a multinomial probability error structure, included in models that are based on maximum likelihood estimation, more closely follows the variability associated with the sampled landing data than does a lognormal error structure used in models based on least squares estimation. Weighted nonlinear regression analysis is used to determine the specific multinomial distribution (sample size $n$ ) that provides the most accurate description of the actual variability associated with the sample estimates of age composition. Implications for stock assessment modeling are discussed. Finally, a linear regression model is derived that describes the relationship between multinomial sample size and the number of boat trips sampled, in efforts to provide an adequate error structure for the models without having to rely on the relatively complex and tedious sampling estimators and subsequent analytical techniques. 


\section{Introduction}

In recent years, stock assessment models have gained widespread application in fisheries management. In particular, several age-structured assessment methods have become the primary tools used to derive estimates of fishery parameters, such as fish population abundance and exploitation rates, in many fisheries throughout the world (Megrey 1989). The motivation behind these assessment methods is that a time series of catchat-age data for a fishery (i.e., estimates of age composition of the landings for a particular stock) can be utilized in models containing certain biological processes of fish populations, such as growth, mortality, and reproduction. The extent to which these models can describe or predict the inherent stochastic properties of an animal population is based largely on the validity and reliability associated with their parameters and assumptions (Pielou 1977, Gulland 1983, Gottelli 1995).

It is generally agreed that estimates of catch-at-age alone are insufficient to reliably determine the status of exploited fish stocks (Doubleday 1976, Pope 1977, Megrey and Wespestad 1988, Quinn and Collie 1990). The types and function of auxiliary data in fishery models need to be examined rigorously to safeguard against inappropriate application in management situations. In particular, it is critical that important model assumptions be reviewed and tested to ensure that generated results are interpreted appropriately (Gudmundsson 1986, Edwards and Megrey 1989, Schnute 1989, Sampson 1993).

To date, one of the most important advances in model development has been the inclusion of an error structure to address the variability associated with the: (1) separate biological processes that influence fish population abundance, such as reproduction and mortality; and (2) input data on which the models are based, namely the sample estimates of catch-at-age (see Megrey 1989) for an extensive review of age-structured stock assessment models). The age-structured assessment models that accommodate stochastic data can be broadly classified into two groups based on the statistical estimation technique that is used (Kimura 1989): (1) the method of least squares (e.g., Doubleday 1976; Pope and Shepherd 1982; Deriso et al. 1985, 1989; Kimura 1989); or (2) the method of maximum likelihood based on multinomial distribution probabilities (e.g., Fournier and Archibald 1982; Dupont 1983; and Methot 1989, 1990). Note that the least squares and maximum likelihood methods generate equivalent solutions if the error terms are assumed to be distributed as normal random variables (Bain and Engelhardt 1987). In addition, although the two groups of models above are most often defined by distinct error structures, each estimation method (nonlinear least squares and maximum likelihood) is capable of fitting lognormally and multinomially distributed catch-at-age data (Kimura 1990). If least squares residuals are weighted inversely by their expected variances, the least squares method can be used in an iterative manner to generate maximum likelihood estimates (McCullagh and Nelder 1989). 
An important theoretical consideration when choosing an appropriate stock assessment model involves defining the correct sampling distribution for estimates of age composition (Kimura 1990). The focus of the work described here is the assumed error structure for age composition data relied upon in the different models, which is one criterion that can be used to differentiate the two groups of models discussed above. That is, the objective of this research does not directly involve a critical examination of the methods of estimation utilized by the different models. Also, note that there exist other sources of error (uncertainty) associated with age-structured stock assessment models that are not evaluated in this paper, including measurement of: (1) aging error (i.e., error associated with determining the age of a fish specimen); (2) effort error; (3) spawnerrecruit error; (4) stochastic mortality; and (5) total catch, including discard and bycatch estimates (Megrey 1989).

The suite of models that is based on least squares estimation generally assumes that observation errors in catch-at-age data are lognormally distributed and the models use $\log _{e}$ transformed estimates of catch-atage. The least squares estimators assume constant variance of the log transformed estimates of catch-at-age, which dictates that the coefficients of variation (CVs) associated with the untransformed catch-at-age estimates be approximately equal (Kimura 1989, 1990; Methot 1990).

In the models that use maximum likelihood estimation, the error structure for catch-at-age data is based on multinomial probabilities, which implies that the CVs associated with the estimates of proportion-at-age are distributed in a multinomial fashion (Methot 1990). That is, the magnitude of the CV decreases steadily as the size of the proportion increases (Fig. 1). The sampling variance of a proportion derived from a multinomial distribution is treated as a binomially defined parameter and calculated as $V(P) \cong[P(1-P) / n]$, where $P$ is the population proportion and $n$ is the number of units in the sample. The CV of $P$ is calculated as $(\sqrt{V(P)} / P)$. In probability theory, $n$ can be practically interpreted as an index that defines a particular distribution from an infinite number of possible probability distributions. In stock assessment models, $n$ represents the sample size associated with an estimated age composition. It is a weighting factor, such as $J_{y}$ below, that adjusts the theoretical $C V$ versus $P$ curve upward or downward to reflect the estimated variability associated with the agecomposition sample. Henceforth, the term "multinomial index" is used to identify a specific $n$ from the family of possible distributions.

The general form of the objective function used in the two groups of models to derive fishery-related parameters is as follows, summation being over $y=1, \ldots, Y$ for years and $a=1, \ldots, A$ for ages: (1) least squares (Deriso et al. 1985), choose parameters that minimize

$$
\sum_{y=1}^{Y} \sum_{a=1}^{A}\left[\log _{e}\left(c_{y a}\right)-\log _{e}\left(\hat{c}_{y a}\right)\right]^{2} ; \text { and }
$$




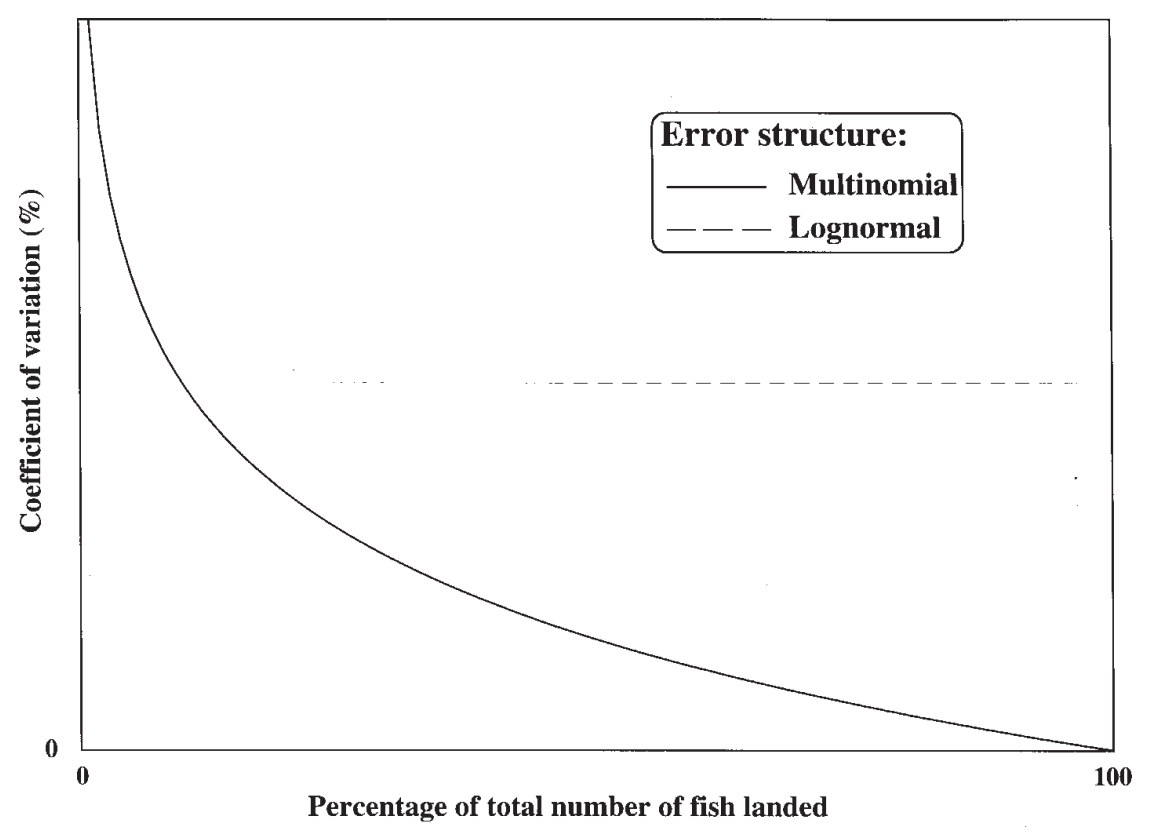

Figure 1. Distributions of estimated coefficients of variation (\%) associated with estimates of age composition (percentage of total number of fish landed) for two different error structure assumptions used in fish stock assessment models. Lines depict the general form of the distributions and do not reflect the inherent variability associated with samples of age composition.

(2) maximum likelihood (Methot 1990), choose parameters that maximize

$$
\sum_{y=1}^{Y} \sum_{a=1}^{A} J_{y} p_{y a} \log _{e}\left(\hat{p}_{y a}\right)
$$

where $c_{y a}$ is the observed catch-at-age (in number), $\hat{c}_{y a}$ is the predicted catch-at-age (in number), $J_{y}$ is a weighting factor that reflects the number of fish in the sample if the fish were selected as a single simple random sample (i.e., if the multinomial probability distribution was strictly correct), $p_{y a}$ is the observed proportion-at-age, and $\hat{p}_{y a}$ is the predicted proportionat-age. The assumption regarding the pattern of variability exhibited by the actual sample estimates of catch-at-age is generally different between these two groups of models (Fig. 1). 
The primary objective of this research was to examine the statistical properties associated with estimates of age composition for groundfish landings in Oregon from 1989 to 1994. Specifically, we graphically present the distributions of estimated CVs associated with estimates of age composition for five species of groundfish to evaluate the appropriateness of the error structure assumed in fishery models to describe the uncertainty associated with the catch-at-age sample data. In addition, we present a nonlinear regression technique and generally discuss other methods that can be used to determine a specific multinomial index $(n)$ to apply to particular age-composition data sets. Finally, a simple linear regression model is developed that describes the relationship between the multinomial indices and the number of boat trips sampled.

\section{Methods}

Estimates of age composition (in number and percent) and their errors were calculated for five species of groundfish commercially landed at Oregon ports from 1989 to 1994: widow rockfish (Sebastes entomelas), yellowtail rockfish (Sebastes flavidus), canary rockfish (Sebastes pinniger), English sole (Pleuronectes vetulus), and Dover sole (Microstomus pacificus). Age-composition data sets for 22 combinations of species and year were analyzed in this study, i.e., age-composition samples for 8 speciesyear combinations were not collected or had specimens that had not been analyzed for age determination at the time of this study. The analyses of age composition for this study were based on a stratified two-stage random sampling design combined with poststratification (Fig. 2). The sampling design used for 1989 and 1990 landings incorporated a single sampling unit (basket of fish) at the second stage and utilized straightforward ratio estimation techniques to derive landing statistics (Sen 1986). A "complete" multistage design was used for 1991-94 landings, which incorporated replicate sampling units at the second stage and used standard two-stage estimators to generate landing estimates (Crone 1995).

A coefficient of variation (CV), (standard error / estimate), was used to describe the variability associated with the individual landing estimates of age composition. This statistic is also referred to as a relative standard error (Som 1973) and a coefficient of variation of the estimate (Cochran 1977). Weighted nonlinear least squares regression (Neter et al. 1990) was applied to estimates of age composition and their estimated CVs to determine multinomial indices $(n)$ for combinations of species and year, with estimated proportions-at-age used as the weights. Simple linear regression methods (e.g., Draper and Smith 1981; Neter et al. 1990) were applied to relate the multinomial indices with the number of boat trips sampled. The number of boat trips represents the first-stage sample size in estimators 


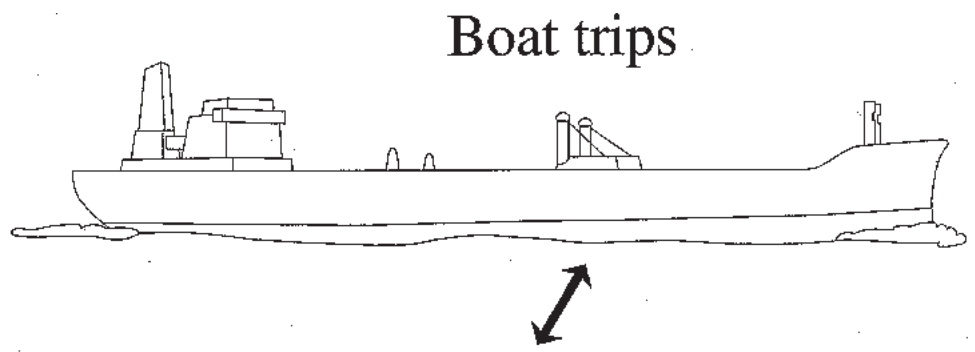

\section{Market categories}

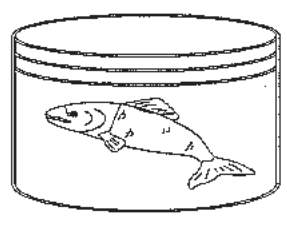

Widow rockfish

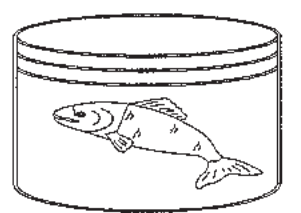

Yellowtail rockfish

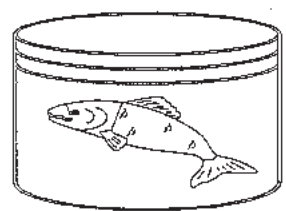

Dover sole

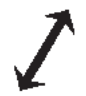 \\ 25 or $50 \mathrm{lb}$ Baskets of fish}
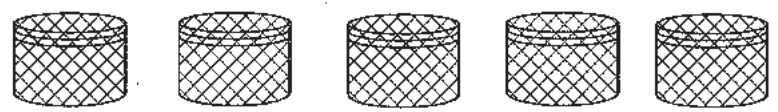

Figure 2. Two-stage sampling design used to monitor age compositions of commercially landed groundfish in Oregon (1989-1994). Port and quarter combinations were treated as strata. Three examples of market categories (presorted groups of fish) are presented. Top: primary sampling units; middle: poststratification units; bottom: secondary sampling units.

associated with the multistage sampling design used to generate age-composition statistics (Fig. 2).

\section{Results}

Results from age-composition analyses were very consistent for the five species evaluated in this study. Selected species-year combinations are presented as examples that generally describe the statistical properties of the age compositions.

The relationship between the individual estimates of age composition, presented as percentages of the total number of fish landed, and 
their associated CVs was negatively curvilinear for each species-year combination (e.g., 1991 results are presented in Fig. 3). The actual ages associated with the data points are not included on the graphs; however, particular data points (ages) are identified to highlight general patterns exhibited in the age compositions. Also, for purposes of graphical clarity, we have omitted age groups that constituted less than $0.07 \%$ of the total landings; the CVs associated with these age groups were very consistent across species and years, ranging from 70 to $110 \%$. In general, estimates that composed large percentages of the total landings were measured with higher relative precision (i.e., had smaller CVs) than estimates of age composition that constituted small percentages of the total landings.

For example, 851,558 age-6 English sole were landed in 1991, which was roughly $34 \%$ of the total number of English sole landed for the year, and the CV associated with this estimate was $13 \%$; whereas, 16,071 age-16 fish were landed, which was approximately $1 \%$ of the total number landed, and this estimate had a CV of $87 \%$ (star-filled squares, middle right panel of Fig. 3).

The CVs associated with the landing estimates clearly mimicked the curves generated from the theoretical multinomial distributions, presented in the figures for $n=100,400$, and 1,000. The role of $n$ as weighting factors in stock assessment models that incorporate an error structure based on multinomial probabilities is discussed below. Although the patterns of variation that characterized the estimated age compositions were generally similar between the five species, the amount of statistical "noise" associated with each set of estimates (species-year combinations) was not identical. For example, ages 7,14 , and 15 made up roughly 5\% (approximately 47,000 fish) of the total landings of canary rockfish in 1991; however, the CVs associated with these similar landing estimates ranged from 17 to $33 \%$ (star-filled squares, middle left panel of Fig. 3). For Dover sole, ages 15 and 16 individually composed approximately $5 \%$ (roughly 1,000,000 fish) of the total landings and these estimates had CVs that were less than 12\% (star-filled squares, lower left panel of Fig. 3).

The theoretical distributions more accurately reflected the variation associated with the estimates of age composition that contributed significantly to the total landings than they did for estimates that constituted small percentages of the total, particularly estimates that composed less than $1 \%$ of the total. For example, the estimate of 759,068 (approximately $18 \%$ ) age- 7 widow rockfish landed in 1991 had a CV of $8 \%$ (star-filled square, upper right panel of Fig. 3), with CVs of 21,11 , and $7 \%$ associated with the analogous percentage defined by the multinomial indices for $n=100$, 400 , and 1,000 , respectively. Whereas, the estimate of 3,634 (roughly $0.09 \%$ ) age-31 widow rockfish had a CV of 59\% (star-filled square, upper right panel of Fig. 3), compared with CVs of 333, 167, and 105\%, for the three theoretical distributions defined by $n=100,400$, and 1,000, respectively.

In general, at least three-fourths of the total landings of a species and year combination was composed of a relatively small range of consecu- 


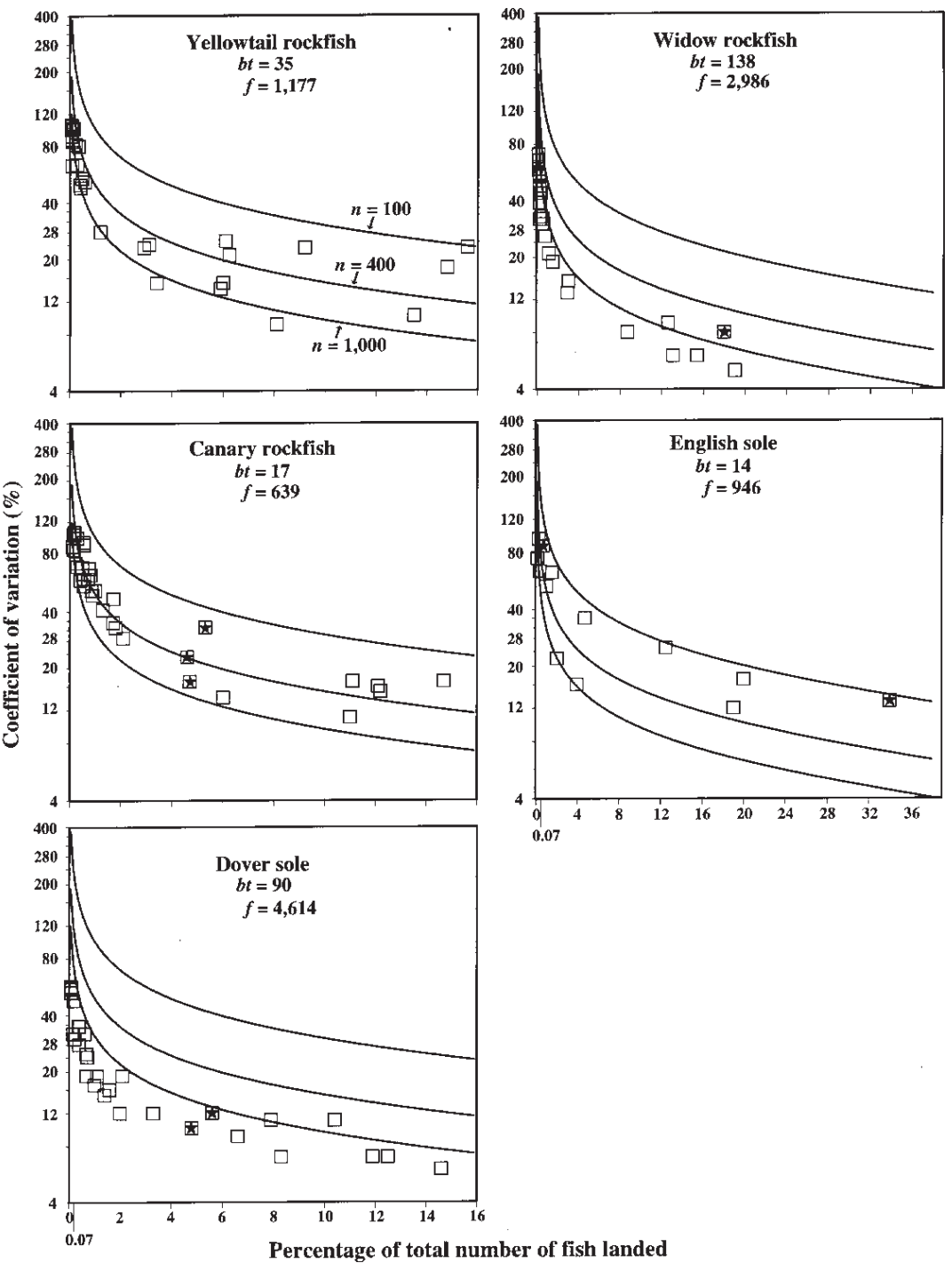

Figure 3. Distributions of coefficients of variation (\%) associated with estimates of age composition (percentage of total number of fish landed denoted by squares) for five species of groundfish landed at Oregon ports in 1991. See Results section for description of star-filled squares. Coefficients of variation derived from percentage estimates for three multinomial indices are presented, $n=100,400$, and 1,000. Estimates for ages that composed at least $0.07 \%$ of the total landings are included. The Y-axis has been logarithmically scaled. For each species, the sample sizes (number of boat trips) used to derive age-composition statistics are denoted as bt and the total number of fish collected across all boat trips is denoted as $f$. 
tive ages that individually contributed at least $5 \%$ to the total and these estimates were relatively precise, with CVs less than $25 \%$. The remaining approximately one-fourth of each age composition included comparatively more ages that individually composed less than $5 \%$ of the total and these estimates were more variable than for the ages that constituted the three-fourths majority, with CVs generally greater than $30 \%$ and most often between 50 and 100\%. Detailed landing statistics for yellowtail rockfish in 1990 illustrate the general properties of the age-composition sample data collected in Oregon from 1989 to 1994 (Table 1).

Weighted nonlinear regression analysis was used to identify the multinomial index $(n)$ that most accurately characterized the actual variation associated with estimates of age composition for combinations of species and year, with weights equal to the estimated proportions-at-age. In effect, ages that were landed more frequently and reflected relatively large proportions of the total landings were given more importance in the fitting procedures than those ages that were associated with small proportions. For example, using this straightforward analytical technique, the age composition of yellowtail rockfish landed in 1991 (upper left panel of Fig. 3) was characterized by a multinomial index with an $n$ of 688.

Following the determination of multinomial indices for age compositions by species and year, a linear regression model was developed that describes the relationship between the multinomial indices and the corresponding number of boat trips sampled for the species-year data sets. The amount of variation associated with a species-year age composition is directly influenced by the number of boat trips sampled (Fig. 2; Crone 1995). A relatively simple model was developed that accounted for a significant portion of the variability $\left(r^{2}=0.90\right)$ in the dependent variable $(n)$, where the number of boat trips sampled was statistically related $(P<0.001)$ to the multinomial index (Fig. 4). The total number of fish that constituted a species-year age-composition sample was another sampling descriptor that was considered as a potential independent variable in the model. Although this variable was found to be a statistically significant $(P<0.001)$ term when included in the regression model along with the number of boat trips sampled, we felt its presence in the model was not applicable, given: (1) it did not substantially increase the amount of total variation in multinomial index $(n)$ accounted for by the regression equation $\left(r^{2}=0.94\right)$; and more important, (2) the strong correlation (multicollinearity) that existed between the two independent variables $(r=0.80)$ confounds the interpretation and use of a fitted regression model (Neter et al. 1990).

\section{Discussion}

Results presented here indicate that stock assessment models that utilize maximum likelihood estimation techniques with a multinomial probability error structure (e.g., Fournier and Archibald 1982; Methot 1989, 1990) more adequately address the variability associated with observed catch- 
Table 1. Age-composition estimates for yellowtail rockfish landings in Oregon (1990).

\begin{tabular}{|c|c|c|c|}
\hline Age & $\begin{array}{l}\text { Landing } \\
\text { estimate }\end{array}$ & $\begin{array}{l}\text { Percent of } \\
\text { total landings }\end{array}$ & CV (\%) \\
\hline 4 & 236 & $<1$ & 102 \\
\hline 5 & 23,759 & 2 & 45 \\
\hline 6 & 140,419 & 11 & 33 \\
\hline 7 & 132,329 & 10 & 18 \\
\hline 8 & 81,400 & 6 & 18 \\
\hline 9 & 135,648 & 10 & 15 \\
\hline 10 & 142,835 & 11 & 10 \\
\hline 11 & 96,412 & 7 & 15 \\
\hline 12 & 101,234 & 8 & 14 \\
\hline 13 & 89,401 & 7 & 18 \\
\hline 14 & 102,838 & & 25 \\
\hline 15 & 74,107 & 6 & 13 \\
\hline 16 & 62,956 & 5 & 22 \\
\hline 17 & 37,099 & 3 & 26 \\
\hline 18 & 21,001 & 2 & 32 \\
\hline 19 & 13,310 & 1 & 28 \\
\hline 20 & 6,461 & $<1$ & 39 \\
\hline 21 & 10,613 & 1 & 32 \\
\hline 22 & 1,142 & $<1$ & 55 \\
\hline 23 & 8,852 & 1 & 42 \\
\hline 24 & 1,000 & $<1$ & 78 \\
\hline 25 & 1,457 & $<1$ & 86 \\
\hline 26 & 1,464 & $<1$ & 71 \\
\hline 27 & 6,556 & $<1$ & 44 \\
\hline 28 & 6,479 & $<1$ & 77 \\
\hline 29 & 3,134 & $<1$ & 52 \\
\hline 30 & 2,911 & $<1$ & 81 \\
\hline 31 & 2,634 & $<1$ & 77 \\
\hline 32 & 90 & $<1$ & 102 \\
\hline 34 & 1,638 & $<1$ & 78 \\
\hline 36 & 701 & $<1$ & 100 \\
\hline 37 & 393 & $<1$ & 101 \\
\hline 38 & 392 & $<1$ & 101 \\
\hline 40 & 43 & $<1$ & 103 \\
\hline 41 & 2,786 & $<1$ & 75 \\
\hline 42 & 1,638 & $<1$ & 78 \\
\hline 46 & 1,245 & $<1$ & 98 \\
\hline Total & $1,316,613$ & 100 & \\
\hline
\end{tabular}

Landing estimates are in number of fish and are based on a sample of 35 boat trips. 


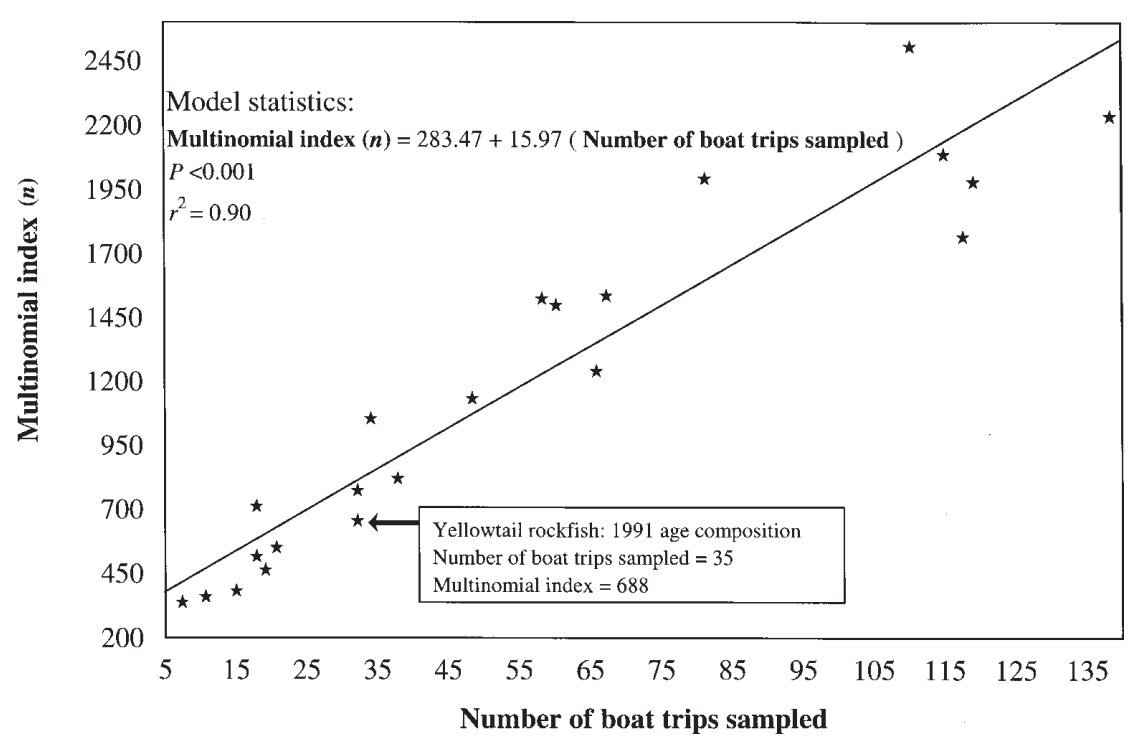

Figure 4. Multinomial index (n) as a linear function of the number of boat trips sampled. The multinomial index (dependent variable) represents a theoretical sample size ( $n$ ) associated with multinomially distributed proportion-at-age estimates. The number of boat trips sampled (independent variable) represents the actual sample size associated with sampling estimators used to derive the age-composition landing statistics. Data points $(\star)$ represent multinomial indices associated with age-composition data sets for combinations of species and year; an example is shown for the age composition of yellowtail rockfish landed in 1991 (see upper left panel of Fig. 3 for estimates of age composition and their CVs for yellowtail rockfish landings in 1991).

at-age data than models based on lognormal measurement errors. The statistics generated from these analyses show clearly that the CVs associated with individual estimates of age composition are not constant, or even approximately so, but rather follow the general properties of a multinomially distributed variable.

The choice of the most appropriate multinomial index $(n)$ to use in a model is inherently problematic. The sampling designs generally employed to collect fishery-related data, along with the selection protocols utilized in the field, generate age-composition estimates that necessarily depart, to some degree, from a strict theoretical probability distribution, multinomial or otherwise. That is, the catch-at-age data used in stock assessment models are most often based on a sample that consists of many boat trips, which have been selected over a period of time using nominally random 


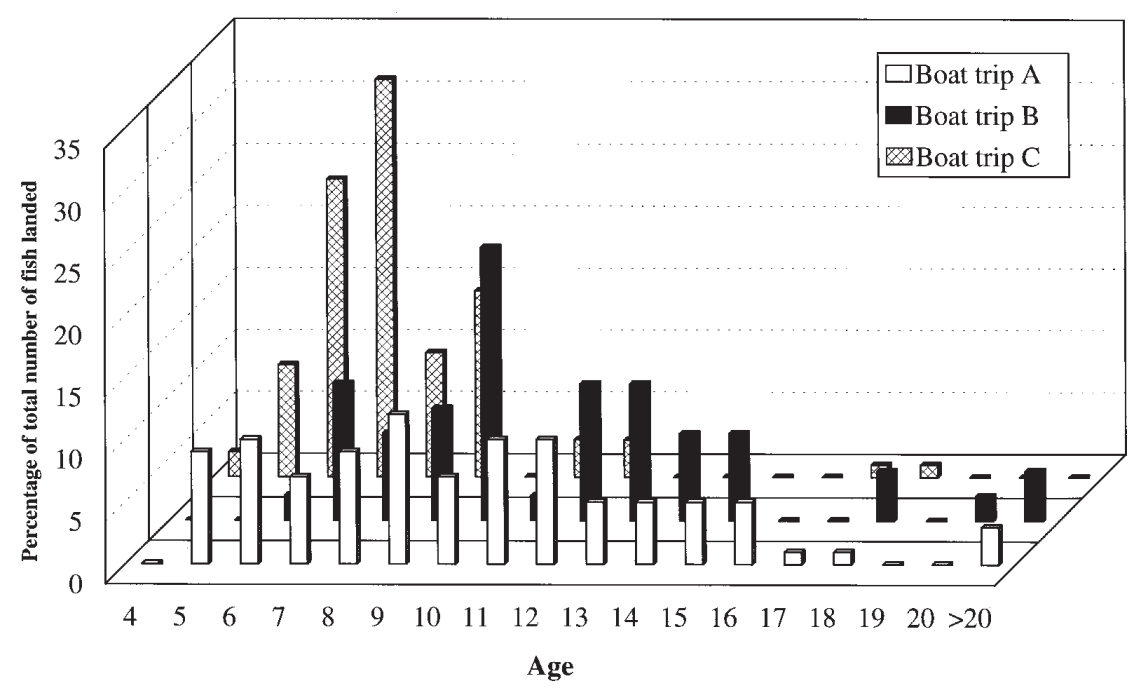

Figure 5. Estimated age compositions (in percent) for landings (in number) of Dover sole for three boat trips $(A, B$, and $C$ ) sampled at Oregon ports in 1994. Sampling design used to generate estimates is described in Crone (1995).

selection protocols (e.g., see Tomlinson 1971, Crone 1995), rather than a sample that was obtained in a strictly random manner from a single trip.

For example, multinomial distribution theory could be applied to a single random sample from a boat trip in a generally straightforward fashion, say to calculate the variance associated with the estimated proportion of age- 6 yellowtail rockfish in the landings. However, the actual estimated variances associated with the landing estimates (in number or percent) were necessarily derived from appropriate sample estimation techniques that required no assumptions regarding distribution properties of the measurement variables. Kimura (1990) also argues that although rigorous multinomial sampling for catch-at-age data may be convenient from an intuitive or modeling standpoint, it is most often an impractical sample selection approach, given the expanse and dynamics of commercial fisheries.

Determination of the most appropriate multinomial index $(n)$ becomes problematic because the age compositions estimated from the individual trips sampled are often considerably different from one another (Fig. 5), which confounds the use of explicit theory (e.g., a multinomial distribution supposition) to summarize the actual variability associated with the sample estimates. In the context of generalized linear modeling, this sampling scenario often results in "overdispersion" in the data, i.e., the vari- 
ability associated with the landing estimates is greater than what is expected from a simple multinomial variable.

Models that account for overdispersion, sometimes called "mixed" models, can be adapted for multistage sampling designs, which requires estimating the scale of overdispersion from a residual deviance statistic (Francis et al. 1993). For example, a comparative study showed that a compound multinomial model was found to be more flexible than a simple multinomial model for defining the variance structure of combined length frequency samples from commercial cod landings; however, the authors advised that this mixed model be applied with caution until further research has fully established the estimation theory (Smith and Maguire 1983). The beta-binomial distribution has also proved useful for specifying and fitting a model that accounts for overdispersion (Crowder 1978). McCullagh and Nelder (1989) suggest an ad hoc approach for dealing with overdispersion that utilizes residual deviance statistics and is directly related to standard regression procedures.

We have shown that the specific form of the multinomial distribution error structure can be estimated using some of the general properties of commercial fishery sampling designs. The motivation behind the techniques we have presented is generally addressed in Shepherd and Nicholson (1986, 1991). That is, Shepherd and Nicholson (1991) present an intuitively attractive ad hoc method to determine an error structure for multiplicative modeling of catch-at-age data, but this method attempts to define a variance (error) structure for landing estimates of age composition in general and does not allow for an objective determination of an appropriate weighting factor. Although general methods, such as the approach proposed by Shepherd and Nicholson, do provide results that are broadly appropriate, the actual catch-at-age data may be much more or less precise than the results indicate, due largely to the effectiveness of the sampling design. We present methods that can be utilized to determine the most appropriate weighting factor $(n)$ to describe the variation associated with a specific age composition(s), thus these methods require that the actual variance measures associated with the sample estimates be available.

Research objectives that address the impact of various error structures on model results have received sparse attention in fishery science (Megrey 1989). Intuitively, a model should be designed in a manner that allows the correct stochastic properties of the catch-at-age data to be incorporated, otherwise additional sources of bias are inherently introduced into the analytical processes. Methot (1990) suggested that the multinomial error structure is a preferred model feature because it emphasizes the variation associated with landing estimates that reflect large proportions of the total landings, which are documented here as being comparatively more precise than the estimates that constituted small proportions of the total. An experiment conducted to examine the sensitivity of assessment results to the assumption of constant selectivity showed that 
the assumed error structure could have a large impact on the final estimates generated from two different modeling approaches, namely stock synthesis analysis and Catch AGE ANalysis or CAGEAN (Sampson 1993).

In contrast, Deriso et al. (1985) demonstrated that a stock assessment model that utilized a least squares estimator (CAGEAN) generated similar results in a comparative study of three different theoretical distributions, based on lognormal measurement error, multinomial measurement error, and process error, applied individually to the model to address the stochastic properties of hypothesized catch-at-age data. Kimura (1990) simulated catch-at-age data using lognormal and multinomial error structures and then analyzed the data using nonlinear least squares and multinomial maximum likelihood estimation, and showed that the results from the overall analyses were similar. The author did however recommend that caution be used when interpreting his findings, given that the results from the simulation experiments may depend strongly on the population and constraints utilized in the model.

Further research is needed that focuses on the relationship between departures from assumptions and model output to critically examine the issue of statistical robustness of fishery models. Results presented here indicate that more complex error structures may be needed to account for the variability in catch-at-age sample data from commercial fisheries, and subsequently, methods need to be developed for incorporating these complicated error structures in the modeling process. The results and discussion presented here are used to evaluate the appropriateness of an assumption used in current age-structured assessment models to address the stochastic properties of catch-at-age data and should not be interpreted as broad recommendations of the overall performance of a model, given that these models incorporate a host of estimated parameters and other assumptions that were not investigated in this study.

\section{Acknowledgments}

This research was supported by: the Oregon Department of Fish and Wildlife, Marine Resources Division, Newport, Oregon (ODFW); grant no. NA89AA-D-SG108 (project no. R-ES-16) from the National Oceanic and Atmospheric Administration (NOAA) to the Oregon State University Sea Grant College Program; and by appropriations made by the Oregon State Legislature. The views expressed herein are those of the authors and do not necessarily reflect the views of NOAA or any of its subagencies. We thank Nick Davies, Terry Quinn, and an anonymous referee for their comments on the manuscript. 


\section{References}

Bain, L.J., and M. Engelhardt. 1987. Introduction to probability and mathematical statistics. Duxbury Press, Boston. 565 pp.

Cochran, W.G. 1977. Sampling techniques, 3rd edn. John Wiley \& Sons, New York. $428 \mathrm{pp}$.

Crone, P.R. 1995. Sampling design and statistical considerations for the commercial groundfish fishery of Oregon. Can. J. Fish. Aquat. Sci. 52:716-732.

Crowder, M.J. 1978. Beta-binomial ANOVA for proportions. Applied Statistics 27:3437.

Deriso, R.B., P.R. Neal, and T.J. Quinn II. 1989. Further aspects of the theory of catch-age analysis. Can. Spec. Publ. Fish. Aquat. Sci. 108:127-135.

Deriso, R.B., T.J. Quinn II, and P.R. Neal. 1985. Catch-age analysis with auxiliary information. Can. J. Fish. Aquat. Sci. 42:815-824.

Draper, N.R., and H. Smith. 1981. Applied regression analysis, 2nd edn. John Wiley \& Sons, New York. 709 pp.

Doubleday, W.G. 1976. A least squares approach to analysing catch at age data. Int. Comm. Northwest Atl. Fish. Res. Bull. 12:69-81.

Dupont, W.D. 1983. A stochastic catch-effort method for estimating animal abundance. Biometrics 39:1021-1033.

Edwards, E.F., and B.A. Megrey. 1989. Mathematical analysis of fish stock dynamics: Rationale and organization of this volume. Amer. Fish. Soc. Symp. 6:1-2.

Fournier, D., and C.P. Archibald. 1982. A general theory for analyzing catch at age data. Can. J. Fish. Aquat. Sci. 39:1195-1207.

Francis, B., M. Green, and C. Payne (eds.). 1993. GLIM 4: The statistical system for generalized linear interactive modeling. Clarendon Press, Oxford. 821 pp.

Gottelli, N.J. 1995. A primer of ecology. Sinauer Associates, Inc., Sunderland, MA. $206 \mathrm{pp}$.

Gudmundsson, G. 1986. Statistical considerations in the analysis of catch-at-age observations. J. Cons. Cons. Int. Explor. Mer 43:83-90.

Gulland, J.A. 1983. Fish stock assessment: A manual of basic methods. John WileyInterscience, New York. 223 pp.

Kimura, D.K. 1989. Variability, tuning, and simulation for the Doubleday-Deriso catch-at-age model. Can. J. Fish. Aquat. Sci. 46:941-949.

Kimura, D.K. 1990. Approaches to age-structured separable sequential population analysis. Can. J. Fish. Aquat. Sci. 47:2364-2374.

McCullagh, P., and J.A. Nelder. 1989. Generalized linear models, 2nd edn. Chapman and Hall, London. 511 pp.

Megrey, B.A. 1989. Review and comparison of age-structured stock assessment models from theoretical and applied points of view. Amer. Fish. Soc. Symp. 6:8-48. 
Megrey, B.A., and V.G. Wespestad. 1988. A review of biological assumptions underlying fishery assessment models. In: W.S. Wooster (ed.), Lecture notes on coastal and estuarine studies: Fishery science and management 28. Springer-Verlag, New York, pp. 31-69.

Methot, R.D. 1989. Synthetic estimates of historical abundance and mortality for northern anchovy. Amer. Fish. Soc. Symp. 6:66-82.

Methot, R.D. 1990. Synthesis model: An adaptable framework for analysis of diverse stock assessment data. Int. North Pac. Fish. Comm. Bull. 50:259-277.

Neter, J., W. Wasserman, and M.H. Kutner. 1990. Applied linear statistical models. Richard D. Irwin, Inc., Boston, MA. 1,181 pp.

Pielou, E.C. 1977. Mathematical ecology. John Wiley \& Sons, New York. 385 pp.

Pope, J.G. 1977. Estimation of fishing mortality, its precision and implications for the management of fisheries. In: J.H. Steele (ed.), Fisheries mathematics. Academic Press, London, pp. 63-76.

Pope, J.G., and J.G. Shepherd. 1982. A simple method for the consistent interpretation of catch-at-age data. J. Cons. Cons. Int. Explor. Mer 40:176-184.

Quinn II, T.J., and J.S. Collie. 1990. Alternative population models for eastern Bering Sea pollock. Int. North Pac. Fish. Comm. Bull. 50:243-257.

Sampson, D.B. 1993. The assumption of constant selectivity and the stock assessment for widow rockfish, Sebastes entomelas. Fish. Bull. U.S. 91:676-689.

Schnute, J. 1989. The influence of statistical error on stock assessment: Illustrations from Schaefer's model. Can. Spec. Publ. Fish. Aquat. Sci. 108:101-109.

Sen, A.R. 1986. Methodological problems in sampling commercial rockfish landings. Fish. Bull. U.S. 84:409-421.

Shepherd, J.G., and M.D. Nicholson. 1986. Use and abuse of multiplicative models in the analysis of fish catch-at-age data. The Statistician 35:221-227.

Shepherd, J.G., and M.D. Nicholson. 1991. Multiplicative modeling of catch-at-age data, and its application to catch forecasts. J. Cons. Cons. Int. Explor. Mer 47:284-294.

Smith, S.J., and J.J. Maguire. 1983. Estimating the variance of length composition samples. Can. Spec. Publ. Fish. Aquat. Sci. 66:165-170.

Som, R.K. 1973. A manual of sampling techniques. Heinemann Educational Books Ltd., London. $384 \mathrm{pp}$.

Tomlinson, P.K. 1971. Some sampling problems in fishery work. Biometrics 27:631641. 


\title{
A Parametric Bootstrap of Catch- Age Compositions Using the Dirichlet Distribution
}

\author{
Erik H. Williams and Terrance J. Quinn II \\ University of Alaska Fairbanks, Juneau Center, School of Fisheries and \\ Ocean Sciences, Juneau, Alaska
}

\section{Abstract}

Stock assessment models that incorporate catch or survey age compositions and indices of abundance are commonly used for managing fisheries. Obtaining error estimates for estimated parameters in these models can be difficult. Many models incorporate a bootstrap procedure for estimating errors. In order to perform a bootstrap procedure, age compositions can be modeled by some type of statistical distribution and are often assumed to follow a multinomial process. However, the multinomial distribution is based on the assumption that a simple random sample of ages is taken from the catch, which in practice is rarely true and can lead to underestimation of error. In some applications, a smaller "effective sample size" is substituted for the actual sample size in a subjective manner. We developed an a posteriori method to model observed age compositions with the Dirichlet distribution, using the estimated age compositions from the stock assessment model. A parametric bootstrap procedure using the fitted Dirichlet distribution provided error estimates for estimated stock assessment parameters. We illustrate this technique by application to an age-structured model for Pacific herring (Clupea pallasi) from Norton Sound, Alaska. The Dirichlet procedure properly accounted for the variation in observed age compositions compared to those estimated by the stock assessment model. The effective sample sizes from the Dirichlet procedure were lower than the actual sample sizes, suggesting that a comparative multinomial bootstrap procedure underestimated the variance.

\section{Introduction}

Fisheries managers commonly utilize stock assessment models which incorporate catch or survey age compositions and indices or estimates of 
abundance (Hilborn and Walters 1992, Quinn and Deriso, in press). These catch-age or age-structured assessment models are among the best techniques for analysis available to fisheries managers. To fully interpret the parameter estimates from catch-age analysis, estimates of parameter variability are required. Obtaining error estimates of estimated parameters in these models can be awkward and sometimes subjective. Many catch-age models incorporate a bootstrap procedure for estimating errors (Efron 1982, Efron and Tibshirani 1993, Quinn and Deriso, in press).

A nonparametric bootstrap procedure, as applied to catch-age analysis, involves randomly sampling age composition residuals with replacement and adding them to the original estimated values, giving a new set of observed values (Deriso et al. 1985). The new observed values are then used to estimate the parameters of the model resulting in a bootstrap replicate. The standard deviation of the bootstrap replicates is an estimate of the standard error of the estimate (Efron 1982, Efron and Tibshirani 1993). In the case of age composition data, large negative residuals relative to the smallest estimated value results in unrealistic negative "observed" values during the bootstrap procedure.

An alternative method of performing a bootstrap of age composition residuals in catch-age analysis which may be more accurate is to fit a parametric model, usually in the form of some type of statistical distribution, to the observed age compositions. The most commonly employed model for age composition data is the multinomial distribution (Fournier and Archibald 1982, Kimura 1990). However, incorporating a multinomial process into a bootstrap procedure may require manipulation of the sample size and assumes simple random sampling with known sample sizes. Fournier and Archibald (1982) and Methot (1986) suggested that there is variability other than that due to multinomial sampling alone. For this reason, they suggested the sample size for estimating age composition be limited to $n=400$, even though the actual sample size is often much larger. In order to accurately apply the multinomial sampling distribution to catch-age analysis, the sample size may be adjusted in an ad hoc fashion so that the resulting error matches the expected level of variance.

Alternatively, an objective method is to determine the error structure empirically by examination of the observed and estimated age compositions. We propose to fit a posteriori the Dirichlet distribution to the set of observed age compositions for implementation in a parametric bootstrap procedure for estimation of standard errors of parameter estimates. We apply this technique and compare it to the multinomial bootstrap procedure using an age-structured model for Pacific herring (Clupea pallasi) from Norton Sound, Alaska (Williams and Quinn 1998).

\section{Methods}

The Dirichlet distribution is a multivariate statistical distribution for a set of random variables that take on values between 0 and 1 , and sum to 1 
(Evans et al. 1993). The beta distribution, a special case of the Dirichlet distribution, for a single variable (or more precisely, two variables summing to 1), is a flexible statistical distribution with two parameters $v$ and $w$. It is appropriate for modeling proportions, because it takes on values between 0 and 1 and its density function can assume many shapes: Ushaped $(v=w, v<1, w<1)$, J-shaped $(v>$ or $<w)$, or unimodal $(v=w, v>1$, $w>1$ ). The probability density function of the Dirichlet distribution for a set of $k$ proportions $\left\{\theta_{a}, a=1, \ldots, k\right\}$ utilizes the gamma function, $\Gamma(\gamma)=\int_{0}^{\infty} \exp (-u) u^{\gamma-1} d u$, and is given by

$$
\frac{\Gamma(\gamma)}{\prod_{a=1}^{k} \Gamma\left(\gamma_{a}\right)} \prod_{a=1}^{k} \theta_{a}^{\gamma_{a}-1} \text {, such that } \sum_{a=1}^{k} \theta_{a}=1 \text { and } \gamma=\sum_{a=1}^{k} \gamma_{a}
$$

We identify $\gamma$ and $\left\{\gamma_{a}, a=1, \ldots, k-1\right\}$ as the parameters of the distribution and obtain $\gamma_{k}$ by subtraction as $\gamma_{k}=\gamma-\sum_{a=1}^{k-1} \gamma_{a}$. Note that $\gamma_{k}$ is not a free parameter of the distribution, as a consequence of the proportions of the distribution summing to 1 .

Let $\theta_{a, t}=$ true proportional age composition at age $a$ in year $t, \theta_{a, t}^{\prime}=$ observed proportional age composition from a sample and $\hat{\theta}_{a, t}=$ estimated proportional age composition from the stock assessment model. Because age composition data are available over several years, the Dirichlet distribution is initially parameterized for each year $t$. The observed proportions, $\theta_{a, t}^{\prime}$, are assumed to follow the Dirichlet distribution with parameters $\gamma_{t}$ and

$$
\gamma_{a, t}=\gamma_{t} \theta_{a, t}, a=1, \ldots, k-1, \text { where } \sum_{a=1}^{k} \theta_{a, t}=1
$$

(For the last age group, $\gamma_{k, t}=\gamma_{t}-\sum_{a=1}^{k-1} \gamma_{a, t}$.)

The parameters $\left\{\gamma_{a,}\right\}$ are assumed proportional to $\left\{\theta_{a, t}\right\}$ in order to connect the data to common parameters across years. The variance of $\theta_{a, t}^{\prime}$ is given by

$$
\begin{gathered}
\operatorname{var}\left(\theta_{a, t}^{\prime}\right)=\gamma_{a, t}\left(\gamma_{t}-\gamma_{a, t}\right) /\left[\gamma_{t}^{2}\left(\gamma_{t}+1\right)\right]=\gamma_{t} \gamma_{a, t}\left[\gamma_{t}\left(1-\theta_{a, t}\right)\right] /\left[\gamma_{t}^{2}\left(\gamma_{t}+1\right)\right] \\
=\theta_{a, t}\left(1-\theta_{a, t}\right) /\left(\gamma_{t}+1\right) .
\end{gathered}
$$

Thus, the Dirichlet distribution variance is related to the variance of proportions, given by $p(1-p) / n$, with $\gamma_{t}=n-1$ and $\theta_{a, t}=p$, where $n$ is the sample size (Zar 1984). Therefore, the parameter $\gamma_{t}$ of the Dirichlet distribution represents an "effective sample size." Intuitively, the $\gamma_{t}$ parameter is inversely related to the amount of variance in the age composition data. 
In this application, the sets of observed and estimated age compositions, $\left\{\theta_{a, t}^{\prime}\right\}$ and $\left\{\hat{\theta}_{a, t}\right\}$, are the only information sources available for estimating the variance. The fit of the Dirichlet distribution to the age composition data is based on the probability density function (1). The observed age compositions, $\left\{\theta_{a, t}^{\prime}\right\}$, from the age-structured model are used in a log likelihood function, for $k$ age groups and $j$ years, given by

$$
\ln L\left(\left\{\gamma_{t}\right\} \mid\left\{\theta_{a, t}^{\prime}\right\}\right)=\sum_{t=1}^{j}\left[\ln \Gamma\left(\gamma_{t}\right)-\sum_{a=1}^{k} \ln \Gamma\left(\gamma_{a, t}\right)+\sum_{a=1}^{k}\left(\gamma_{a, t}-1\right) \ln \left(\theta_{a, t}^{\prime}\right)\right]
$$

with $\gamma_{a, t}=\gamma_{t} \hat{\theta}_{a, t}$ from equation (2). By maximizing (3), estimates of the Dirichlet parameters $\hat{\gamma}_{t}$ and $\left\{\hat{\gamma}_{a, t}, a=1, \ldots, k-1\right\}$ are obtained. (For the last age group, $\hat{\gamma}_{k, t}=\hat{\gamma}_{t}-\sum_{a=1}^{k-1} \hat{\gamma}_{a, t}$.) The larger the discrepancies between the observed and estimated age compositions, the smaller $\hat{\gamma}_{t}$ should be. A possibly more parsimonious model may be warranted in which a single effective sample size $\gamma_{t}=\gamma$ is used.

The parametric bootstrap analysis is performed by using the maximum likelihood estimates, $\left\{\hat{\gamma}_{a, t}\right\}$, to generate Dirichlet distributed random age compositions. Some methods of generating Dirichlet random age compositions are to use the $\hat{\gamma}_{a, t}$ Dirichlet parameters to calculate (1) random gamma variates (using a common scale parameter and $\left\{\hat{\gamma}_{a, t}\right\}$ for the shape parameters), (2) beta variates (with parameters $\left\{\hat{\gamma}_{a, t}\right\}$ and $\left\{\hat{\gamma}_{t}-\hat{\gamma}_{a, t}\right\}$ ) or (3) chi-squared variates (with $\left\{2 \hat{\gamma}_{a, t}\right\}$ degrees of freedom) (Evans et al. 1993). In order to follow the Dirichlet distribution, the random age compositions must be adjusted to sum to one by dividing by the sum of the variates across ages for that year.

The Dirichlet random age compositions are then used in place of the observed age compositions in the model and the parameters re-estimated to produce one bootstrap sample. Efron and Tibshirani (1993) suggest a bootstrap sample size of no less than 1,000 for accurate standard errors and confidence intervals. Figure 1 illustrates the algorithm for fitting the Dirichlet distribution to age composition data and the procedure for bootstrapping the model for error estimates.

The nature of this log-likelihood function does not allow for observed age compositions of 0 or 1 to be used, as seen in the log-likelihood equation (3). If not too many observed proportions of zero occur, then essentially they can be ignored in the likelihood estimation of the $\gamma_{t}$ s. Note that if $\gamma_{a, t}=1$, then its likelihood contribution is zero; this is one of the few cases of the Dirichlet distribution with a nonzero probability for $\theta_{a}=0$. In the bootstrap replications, there is no problem with zeros, because the $\hat{\gamma}_{a, t}$ s are based on estimated proportions $\left\{\hat{\theta}_{a, t}\right\}$ that are usually nonzero. If there are quite a few zeros, then it might be best to pool ages to eliminate the zeros. 


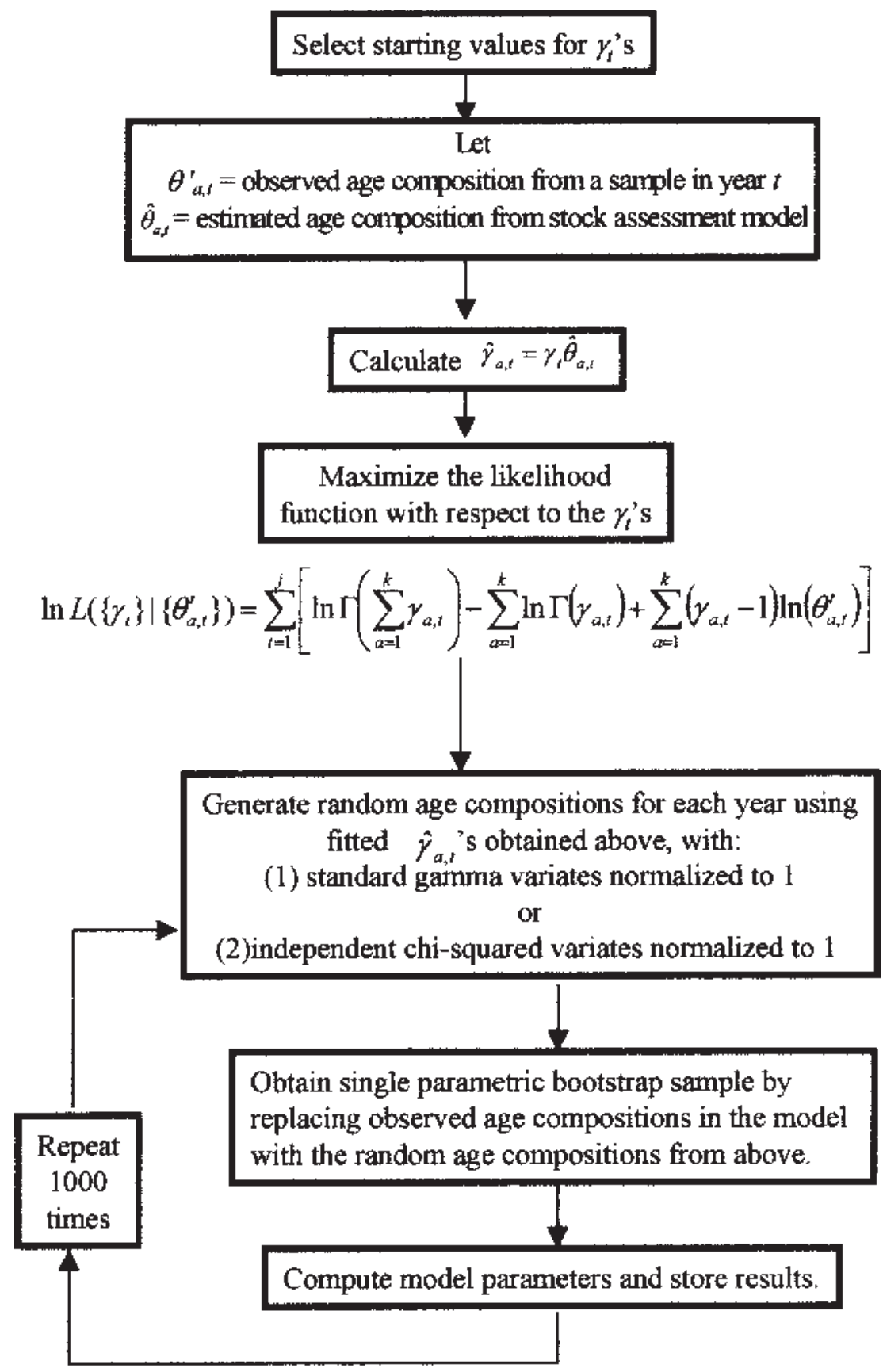

Figure 1. Flow chart of the methodology for applying the Dirichlet distribution bootstrap procedure to an age-structured model with age composition data. 
Table 1. Annual sample sizes for age composition of Norton Sound, Alaska herring.

\begin{tabular}{lrc}
\hline Year & Gillnet fishery & Total run \\
\hline 1981 & 1,084 & 4,244 \\
1982 & 637 & 2,163 \\
1983 & 994 & 3,570 \\
1984 & 671 & 2,056 \\
1985 & 1,265 & 3,550 \\
1986 & 927 & 2,981 \\
1987 & 408 & 1,595 \\
1988 & 388 & 1,100 \\
1989 & 622 & 1,593 \\
1990 & 555 & 2,586 \\
1991 & 914 & 3,704 \\
1992 & 0 & 1,197 \\
1993 & 1,410 & 3,968 \\
1994 & 789 & 2,626 \\
1995 & 1,645 & 6,159 \\
1996 & 1,372 & 4,231 \\
\hline
\end{tabular}

a No fishery occurred in 1992.

\section{Application to Pacific Herring}

We used an age-structured model for Pacific herring from Norton Sound, Alaska developed by Williams and Quinn (1998). This model incorporates catch and total-run age composition data and aerial survey estimates of abundance for years 1981 to 1996 and ages 3 to $10+$, similar to other agestructured models for Pacific herring (Funk et al. 1992, Brannian et al. 1993, Yuen et al. 1994). Sample sizes for the gillnet and total run age compositions were usually large, ranging from about 400 to over 6,000 (Table 1). A method for determining the variability associated with agestructured model estimates for Pacific herring could prove useful for herring management, because no variance estimates are currently calculated by the Alaska Department of Fish and Game (F. Funk, Alaska Department of Fish and Game, personal communication).

We fitted two Dirichlet distributions to the observed catch and totalrun age-composition data: one with constant $\gamma$ for each age composition data set, the other with annual $\gamma_{t}$. Estimates were obtained by maximizing the log likelihood function (3) in an Excel spreadsheet (available from the authors), which utilizes a quasi-Newton optimizer. Estimates of $\gamma$ and $\gamma_{t}$ for the gillnet and total run age compositions are given in Table 2, along with 
Table 2. Maximum likelihood estimates $\gamma$ and $\gamma_{t}$ for gillnet and total run age compositions, along with maximum log-likelihood values.

\begin{tabular}{crc}
\hline & Gillnet & Total run \\
\hline$\hat{\gamma}$ & 21.57 & 26.33 \\
$\hat{\gamma}_{t}$ & & \\
1981 & 7.39 & 17.11 \\
1982 & 41.38 & 17.23 \\
1983 & 21.52 & 24.95 \\
1984 & 22.42 & 45.24 \\
1985 & 29.78 & 25.17 \\
1986 & 11.66 & 42.92 \\
1987 & 22.13 & 19.93 \\
1988 & 19.61 & 18.87 \\
1989 & 22.12 & 91.77 \\
1990 & 34.07 & 80.41 \\
1991 & 86.22 & 20.27 \\
1992 & & 18.71 \\
1993 & 279.01 & 54.35 \\
1994 & 129.28 & 11.57 \\
1995 & 150.37 & 23.74 \\
1996 & 11.51 & 20.23 \\
$\hat{\gamma}_{t}$ & 118.11 & 177.42 \\
$\hat{\gamma}_{t}$ & 137.59 & 182.00 \\
\hline Log likelihood & &
\end{tabular}

log likelihood statistics. The fit using annual $\gamma_{t}$ parameters for the gillnet data set was significantly better than using a constant $\gamma$ (likelihood ratio test: $\chi^{2}=38.97, P<0.001$ ), while the annual $\gamma_{t}$ parameterization for the total run data set was not significantly different $\left(\chi^{2}=9.16, P=0.87\right)$. Note that the estimates of $\gamma$ in Table 2 are smaller than the sample sizes in Table 1 , showing that effective sample size is smaller than actual sample size.

In order to evaluate the annual $\gamma_{t}$ parameterization fits of the Dirichlet distribution, we calculated the empirical standard deviation for each year in each data set. These values are compared to the analytical standard deviation based on the Dirichlet variance formulae given above. Fig. 2 indicates the Dirichlet standard deviation based on the computed effective sample sizes is comparable to the empirical standard deviation from the age-structured model. 

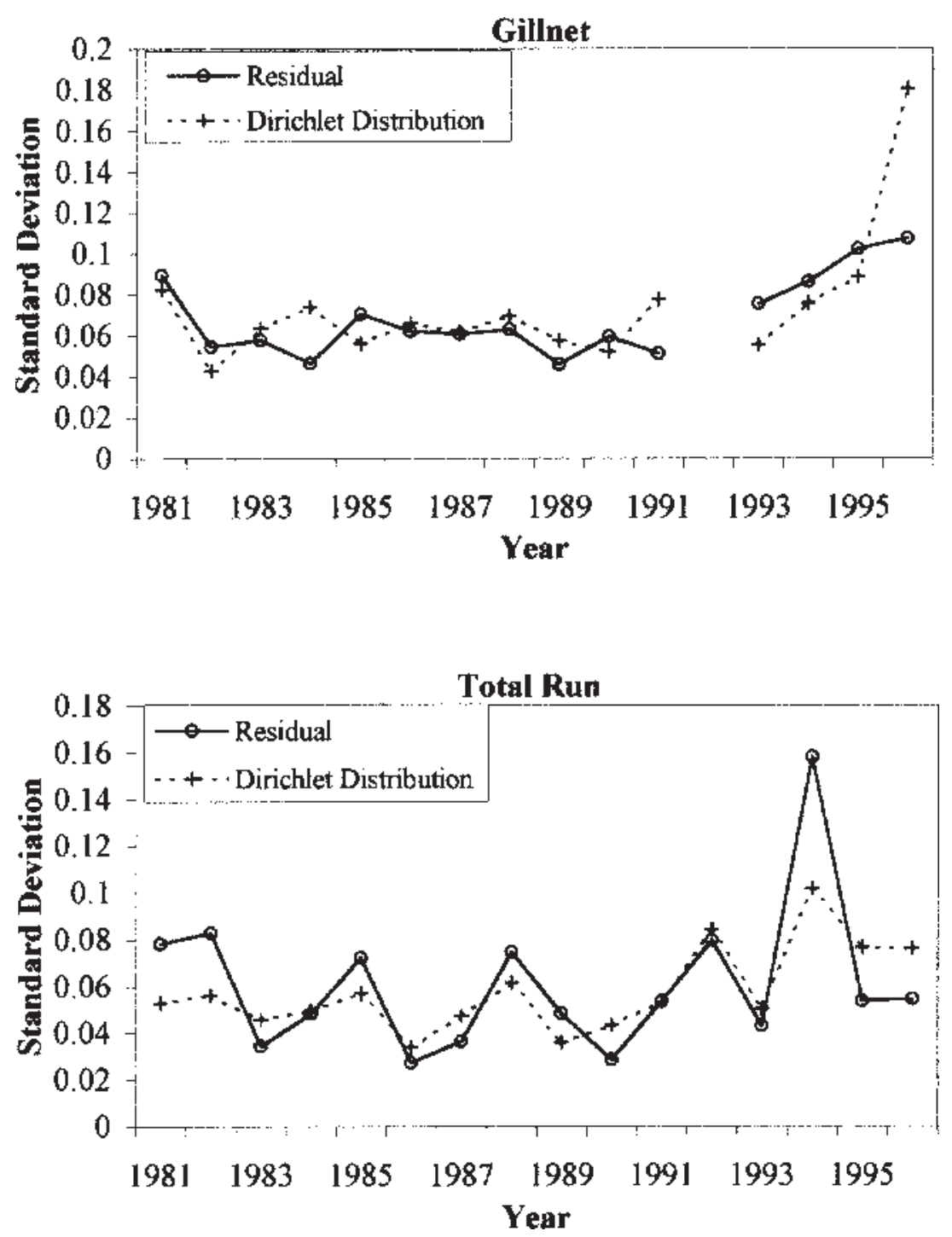

Figure 2. Standard deviations from the residuals and annual $\gamma_{t}$ case of the Dirichlet distribution fit of the Norton Sound herring age-structured model. 
As mentioned earlier, zero-valued observed age compositions cannot be estimated by the Dirichlet distribution. For this application there were some unrepresented (zero) ages, due to a closed fishery in 1992 and selective fishing pressure on older fish. In the bootstrap procedure these values were left at zero. A standard non-parametric bootstrap procedure was used for the aerial survey biomass data. A bootstrap sample of 1,000 was chosen for this exercise.

For comparison to the Dirichlet distribution bootstrap procedure, a multinomial bootstrap procedure was performed with the Norton Sound herring age-structured model using the sample sizes in Table 1. The objective function of the Norton Sound herring model is a simple combination of sums of squares, which does not follow a multinomial process. The multinomial bootstrap procedure was only intended as a comparison to the Dirichlet distribution bootstrap procedure and therefore changing the objective function is not warranted.

\section{Bootstrapping Results}

One of the 1,000 bootstrap samples for the annual $\gamma_{t}$ case of the Dirichlet distribution failed to converge by settling on unrealistic, negative valued estimates. This failure suggests some evidence of model instability but is inconsequential to the final results, so we ignored this replication. All the bootstrap samples for the constant $\gamma$ case of the Dirichlet distribution and for the multinomial distribution appeared to be realistic (no zero or negative valued estimates).

From the bootstrap samples the coefficient of variation ( $c v$, bootstrap standard deviation divided by original estimate), a relative measure of uncertainty, was calculated for estimated total biomass (Fig. 3), pre-fishery abundance estimates for recruitment age herring (age 3) (Fig. 4), and selectivity and catchability parameters in the model (Fig. 5). In general, the multinomial distribution resulted in lower $c v$ estimates as compared to the two Dirichlet distribution estimates, suggesting that use of the multinomial understates actual variability. The error estimates for the total biomass and aerial survey parameters are similar for all three bootstrap procedures, probably because these quantities are determined more from aerial survey information than from age composition information. The $c v$ s for the fishery and sampling gear selectivities indicate larger $c v s$ for the two Dirichlet bootstrap procedures, particularly for the sampling gear selectivity parameters for the last six years. The recruitment $c v s$ indicate the difference among the three bootstrap procedures increases in more recent years. The annual $\gamma_{t}$ case for the Dirichlet bootstrap procedure seems to result in the highest $c v s$ in the most recent years.

\section{Discussion}

The residuals from an age-structured model include both measurement and process error. The true sample size in a multinomial distribution boot- 


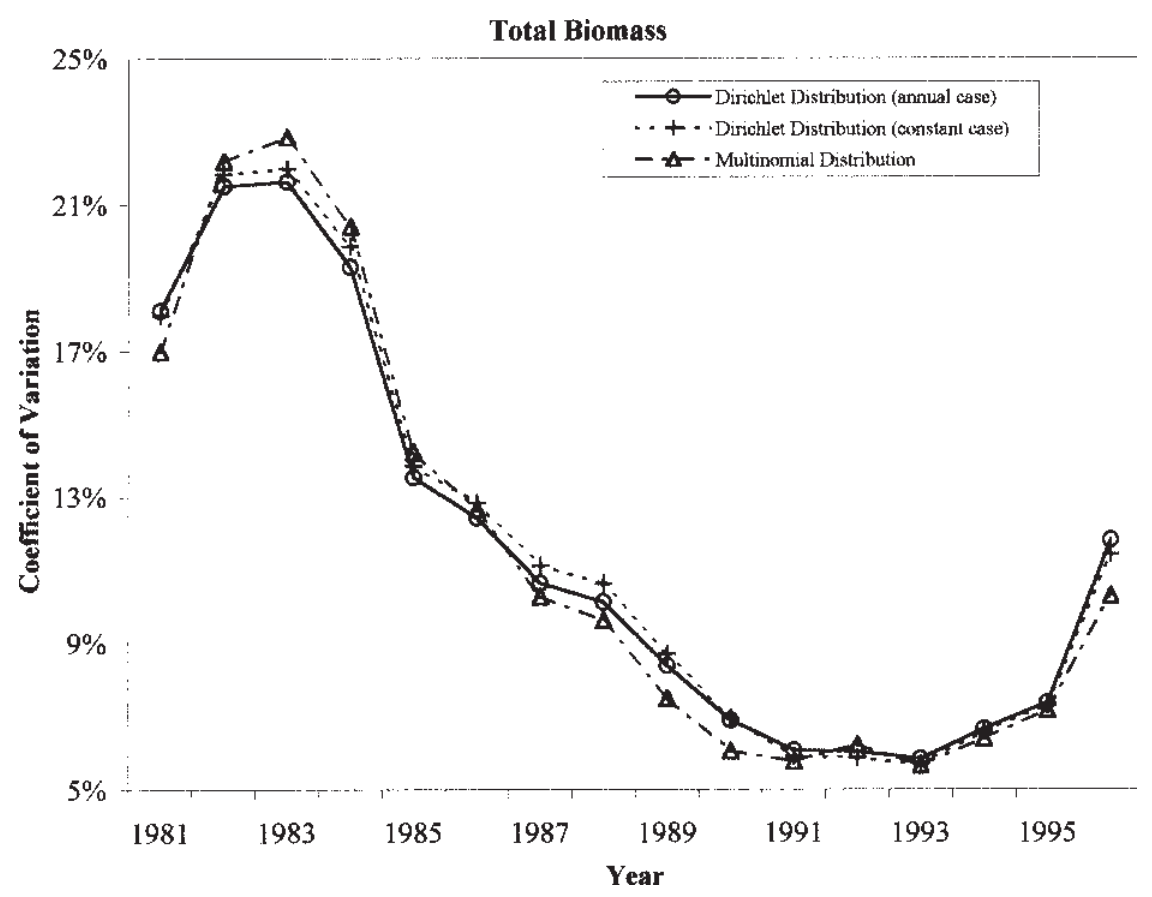

Figure 3. Annual percent error estimates for total biomass from 1,000 bootstrap samples of three different procedures for the Norton Sound herring agestructured model.

strap procedure is limited to representing the measurement error in the model under simple random sampling and has been suggested to be an inappropriate representation of the true estimation error (Fournier and Archibald 1982, Methot 1986). The multinomial bootstrap error estimates in this application are useful for comparison to the Dirichlet distribution bootstrap error estimates. Since the Dirichlet distribution fit is based on the observed and estimated age compositions from the model, the resulting $c v s$ for most cases were higher than those for the multinomial. This result indicates that the Dirichlet distribution is capturing variation in the estimates that is unaccounted for by the multinomial distribution. Otherwise the estimates of effective sample size would have been much closer to the actual ones. The multivariate logistic model is an alternative approach for incorporating greater variability into stock assessment models (Schnute and Richards 1995).

The annual $\gamma_{t}$ case produced higher $c \nu$ s than the constant $\gamma$ case, unexpectedly, since the maximum likelihood result in Table 2 indicated a better fit for the $\gamma_{t}$ case. The reason for this is probably due to the lower 


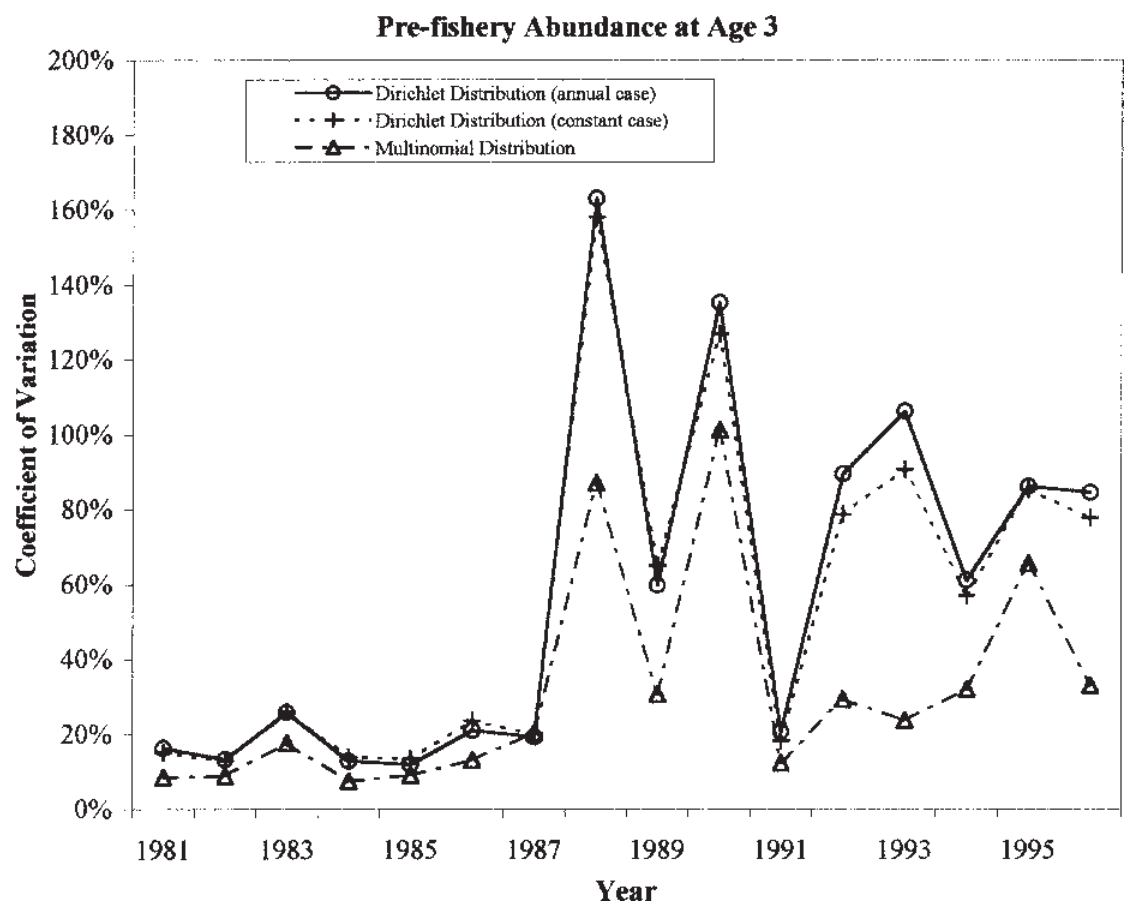

Figure 4. Annual percent error estimates for pre-fishery abundance at age 3, the age of recruitment, from 1,000 bootstrap samples of three different procedures for the Norton Sound herring age-structured model.

effective sample sizes in the last three years for the total run data and the last year for the gillnet data for the annual $\gamma_{t}$ case relative to the constant $\gamma$ case. Since the nature of age-structured analysis is to track cohorts through time, poor fits in the last few years will affect estimates in earlier years. So, even though the constant $\gamma$ case involves less parameterization, it resulted in lower $c v s$ in later years, because it smoothed over the high variability in the last years of the age-structured model.

Coefficients of variation for total biomass and the aerial survey catchability parameters from the Dirichlet method were much closer to the multinomial estimates as compared to the abundance and selectivity parameter estimates. Since the total biomass estimates and catchability parameters depend primarily on the aerial survey abundance data, the similarity of $c \nu$ s for all three bootstrap methods suggests these portions of the model are relatively unaffected by changes in sample size for the age composition data. The similarity of $c \nu$ s is consistent with the aerial survey abundance component in the all three bootstrap procedures being computed by the same non-parametric procedure. This result suggests 


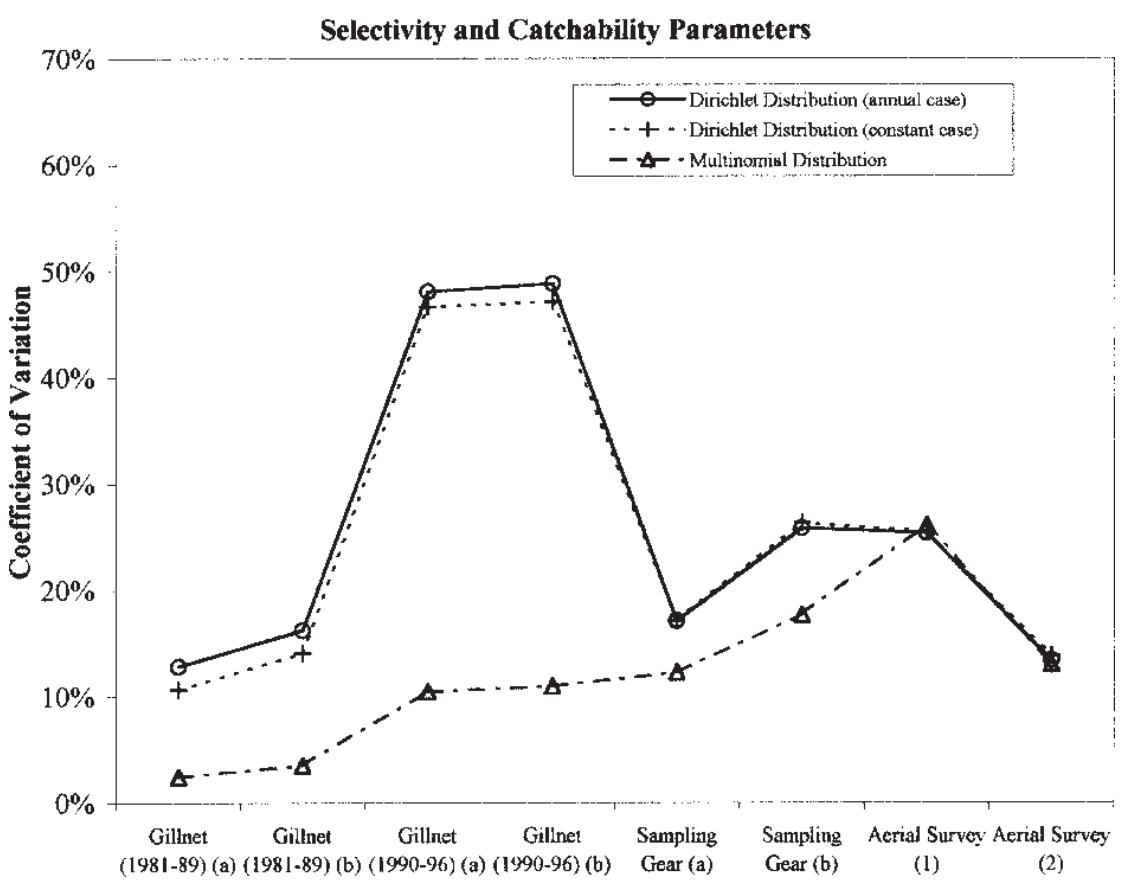

Figure 5. Percent error estimates for gillnet fishery, sampling gear, and aerial surveyability parameters from 1,000 bootstrap samples of three different procedures for the Norton Sound herring age-structured model.

that the recommendation to limit age composition samples to 400 observations (Fournier and Archibald 1982, Methot 1986) in order to prevent age composition from dominating the likelihood does not have a strong rationale. Indeed, since effective sample size can be much smaller than actual sample size, a re-evaluation of sample size requirements in agestructured models is needed.

Overall, the Dirichlet distribution appears to be a statistical distribution which allows for fairly easy and objective bootstrap estimates of error for stock assessment models which utilize age composition data. It provides a means to utilize the observed variation in the model without having to resort to making unrealistic assumptions about the error distribution. An implicit assumption in this approach is that the underlying stock assessment model is correct. If not, the estimated proportions $\left\{\hat{\theta}_{a, t}\right\}$ may be biased, which would lead to bias in the Dirichlet parameter estimates.

We also note that this approach is an approximation to a true bootstrap, in which the original objective function for age composition would 
be based on the Dirichlet distribution. Equations (1) and (3) for the Dirichlet distribution would then be incorporated directly into the objective function of the stock assessment model. Dirichlet parameters would be estimated jointly with the other model parameters, which would avoid the necessity of fitting the Dirichlet distribution a posteriori as we did. Further study is necessary to determine if the additional model complexity presents difficulties in estimation, but such an approach would lend greater consistency to the stock assessment. We did not do so here because we were looking for a quick and simple procedure to generate standard errors after being satisfied with the basic stock assessment results.

Indeed, the major strength of bootstrap procedures in general is their ability to provide measures of uncertainty for all key variables of interest in a straightforward manner and with a minimum of assumptions. Our procedure should be generally applicable to stock assessment models with observed and estimated proportions.

Finally, the concept of effective sample size need not invoke the Dirichlet distribution. An empirical estimate of effective sample size can be made from the observed and estimated age compositions for a given year and gear type (McAllister and Ianelli 1997). If $\theta_{a}^{\prime}$ and $\hat{\theta}_{a}$ are the observed and estimated age compositions for a given year (omitting the time subscript for clarity), then an estimate of the mean squared error is $\sum_{a}^{k}\left(\theta_{a}^{\prime}-\hat{\theta}_{a}\right)^{2} / k$. The average estimated variance of observed proportions from the multinomial distribution is $\sum_{a}^{k} \theta_{a}^{\prime}\left(1-\theta_{a}^{\prime}\right) /(k n)$, where $n$ is sample size. (Alternatively, the estimated $\hat{\theta}_{a}$ s could be used.) By equating these two quantities and solving for $n$, the estimated effective sample size is $n=\Sigma_{a} \theta_{a}^{\prime}\left(1-\theta_{a}^{\prime}\right) / \Sigma_{a}\left(\theta_{a}^{\prime}-\hat{\theta}_{a}\right)^{2}$. A bootstrap procedure could be performed utilizing these effective $n$ values across years with the multinomial distribution. However, the approach utilizing the Dirichlet distribution is more rigorous, and is based on simultaneous analysis of all the information. The Dirichlet approach tends to give lower effective sample sizes, because it explicitly accounts for the extra variance due to the use of observed or estimated proportions.

\section{Acknowledgments}

This publication is the result of research sponsored by Alaska Sea Grant with funds from the National Oceanic and Atmospheric Administration, Office of Sea Grant, Department of Commerce, under grant no. NA90AA-DSG066, project number R/07-16, from the University of Alaska with funds appropriated by the state, the Alaska Department of Fish and Game, Herring Natal Habitats project under Exxon Valdez Oil Spill restoration funds, and a Rasmuson Fisheries Research Center fellowship. The U.S. Government is authorized to produce and distribute reprints for government purposes, notwithstanding any copyright notation that may appear hereon.

We would like to thank Dr. Grant G. Thompson for an excellent review of the beta version of this paper and Dr. Jim Ianelli for helpful suggestions. 
Fritz Funk and Helen Hamner provided the Norton Sound herring fishery and sampling data upon which the age-structured model is based.

\section{References}

Brannian, L.K., K.A. Rowell, and F. Funk. 1993. Forecast of the Pacific herring biomass in Togiak District, Bristol Bay, 1993. Alaska Department of Fish and Game, Regional Information Report 2A93-42, Anchorage.

Deriso, R.B., T.J. Quinn II, and P.R. Neal. 1985. Catch-age analysis with auxiliary information. Can. J. Fish. Aquat. Sci. 42:815-824.

Efron, B. 1982. The Jacknife, the Bootstrap and other resampling plans. SIAM Conf. Ser. 38.

Efron, B., and R.J. Tibshirani. 1993. An Introduction to the Bootstrap. Chapman and Hall, New York. 436 pp.

Evans, M., N. Hastings, and B. Peacock. 1993. Statistical distributions. John Wiley and Sons, Inc., New York. 170 pp.

Fournier, D., and C.P. Archibald. 1982. A general theory for analyzing catch at age data. Can. J. Fish. Aquat. Sci. 39:1195-1207.

Funk, F., L.K. Brannian, and K.A. Rowell. 1992. Age-structured assessment of the Togiak herring stock, 1978-1992, and preliminary forecast of abundance for 1993. Alaska Department of Fish and Game, Regional Information Report 5J9211 , Juneau.

Hilborn, R., and C.J. Walters. 1992. Quantitative fisheries stock assessment: choice, dynamics, and uncertainty. Chapman and Hall, New York. 570 pp.

Kimura, D.K. 1990. Approaches to age-structured separable sequential population analysis. Can. J. Fish. Aquat. Sci. 47:2364-2374.

McAllister, M.K., and J.N. Ianelli. 1997. Bayesian stock assessment using catch-age data and the sampling-importance resampling algorithm. Can. J. Fish. Aquat. Sci. 54:284-300.

Methot, R.D. 1986. Synthetic estimates of historical abundance and mortality for northern anchovy, (Engraulis mordax). Southwest Fish. Center Administrative Rep. LJ 86-29. 85 pp.

Quinn II, T.J., and R.B. Deriso. In press. Quantitative fish dynamics. Oxford Univ. Press, New York.

Schnute, J.T., and L.J. Richards. 1995. The influence of error on population estimates from catch-age models. Can. J. Fish. Aquat. Sci. 52:2063-2077.

Williams, E.H., and T.J. Quinn II. 1998. Age-structured analysis of Pacific herring from Norton Sound, Alaska. Alaska Fish. Res. Bull. 4(2):87-109.

Yuen, H.J., L.K. Brannian, and F. Funk. 1994. Forecast of the Kamishak herring stock in 1994. Alaska Department of Fish and Game, Regional Information Report 2A94-12, Anchorage.

Zar, J.H. 1984. Biostatistical analysis. Prentice-Hall, Inc., Englewood Cliffs, NJ. 718 pp. 


\title{
Some Intrinsic Limitations of Sample Variances in Stock Assessment Models
}

\author{
Clay E. Porch \\ National Marine Fisheries Service, Southeast Fisheries Science Center, \\ Miami, Florida
}

\begin{abstract}
Most modern stock assessment algorithms incorporate multiple catch and abundance estimates, with differing levels of precision. Often these estimates are weighted by their corresponding variances, but estimates of variance are even less precise than estimates of expectation. For this reason, transformations and weighting schemes based on a presumed variance to expectation relationship have often been suggested. Since the true variance to expectation relationship is seldom known, it is usually inferred from regressions of sample variance on sample mean. Unfortunately, the sample variances of non-negative variates like catch per unit effort lie within bounds that are functions of the sample size, sample mean, and maximum possible catch (density). Furthermore, the sample mean and sample variance are self-correlated because the sample mean appears in the expression for sample variance. This paper demonstrates that these, and other mathematical artifices, can force a strong spurious correlation between the sample variance and sample mean regardless of the statistical distribution of the data. Therefore, plots of sample variance against sample mean are unlikely to reveal the true variance to mean relationship for any given population. Moreover, stock assessments that use sample variance to weight the input observations will tend to be biased. Underestimates will tend to receive too much weight and overestimates too little, particularly if the resource in question is uncommon and the sample sizes are small.
\end{abstract}




\section{Introduction}

Most fishery stock assessments depend on estimates of historical abundance derived from resource surveys and samples of the fishery catch. Such data are often expensive to collect, but because of their highly variable nature, tend to yield rather imprecise estimates unless the number of sample observations $(n)$ is large. For this reason, it is important to develop efficient sampling programs that maximize precision under the limited resources available. Of course historical data cannot be remedied by changes in future sampling. One common solution has been to incorporate multiple data sources into the stock assessment, thereby increasing the effective $n$. In that case the different estimates should be weighted according to their precision, either implicitly by a suitable transformation or explicitly by modeling the variances.

Whether one wishes to develop an efficient sampling scheme or properly weight the estimates in a stock assessment model, a central issue is estimating variance. It is well known that if $n$ observations $\left\{x_{1}, x_{2}, \ldots, x_{n}\right\}$ are sampled at random from an infinite population, then the sample mean $(\bar{x})$ and sample variance,

$$
s^{2}=\frac{\sum_{i=1}^{n}\left(x_{i}-\bar{x}\right)}{n-1},
$$

are unbiased estimators of the population mean $\mu$ and population variance $\sigma^{2}$. The variance of $\bar{x}$ is $\sigma^{2} / n$; however, $\sigma^{2}$ is seldom known so $s^{2}$ is often used in its place. Unfortunately, $s^{2}$ is very inefficient (Table 1). More efficient estimators of $\sigma^{2}$ can be derived if the statistical distribution of the population is known, but their performance may be questionable where the data depart from the assumed distribution (see Smith 1990, Myers and Pepin 1991, Pennington 1996). Theoretical arguments supporting one distribution or another abound in the fisheries literature (Taylor 1953, Houser and Dunn 1967, Pella and Psaropulos 1975, Mangel and Beder 1985, Deriso and Parma 1987, Porch and Fox 1990, Pennington 1996), but none apply universally. In any case, neither $s^{2}$ nor its distribution-specific counterparts will in general be a useful proxy for $\sigma^{2}$ unless $n>100$.

An alternative method of specifying $\sigma^{2}$ has its roots in the work of Curtis (1943) and Bartlett (1947), who showed that transformations to homogenize variance can be determined from the relationship between $\sigma^{2}$ and $\mu$. Inasmuch as the $\sigma^{2}$ to $\mu$ relationship is seldom known, it is frequently inferred from regressions of $s^{2}$ on $\bar{x}$ (Green 1979, Taylor 1980, Taylor et al. 1983). This practice is quite common in fisheries work because $s^{2}$ and $\bar{x}$ are often found to be highly correlated (Taylor 1953, Small and Downham 1985, Porch and Fox 1990, Lester et al. 1991, Cyr et al. 
Table 1. Coefficients of variation for $\bar{x}$ and $s^{2}$ with selected probability densities and sample sizes (each computed from 2,000 replicate samples drawn at random with replacement).

\begin{tabular}{lcrrrr}
\hline $\begin{array}{l}\text { Density } \\
\left(\mu, \sigma^{2}\right)\end{array}$ & Statistic & 5 & 10 & 20 & 100 \\
\cline { 3 - 6 } & $\bar{x}$ & 3 & 2 & 2 & 1 \\
Uniform & $s^{2}$ & 51 & 32 & 21 & 9 \\
$(100,57)$ & $\bar{x}$ & 16 & 11 & 8 & 4 \\
Normal & $s^{2}$ & 72 & 46 & 33 & 14 \\
$(20,50)$ & $\bar{x}$ & 24 & 18 & 12 & 5 \\
Lognormal & $s^{2}$ & 132 & 101 & 68 & 29 \\
$(10,30)$ & $\bar{x}$ & 14 & 10 & 7 & 3 \\
Poisson & $s^{2}$ & 71 & 47 & 33 & 14 \\
$(10,10)$ & $\bar{x}$ & 46 & 31 & 22 & 10 \\
Neg. binomial & $\overline{4}$ & & \\
$(5,25)$ & $s^{2}$ & 125 & 82 & 61 & 26 \\
\hline
\end{tabular}

1992, Warwick and Clarke 1993, Welch and Ishida 1993, Dong and Restrepo 1996). However, the relationship between $s^{2}$ and $\bar{x}$ is largely spurious.

Downing (1989) pointed out that the $s^{2}$ of non-negative variates must be less than or equal to $n \bar{x}^{2}$. Tokeshi (1995) argued that "ecological common sense" also dictates $s^{2} \geq \bar{x}$ and that the restricted sampling space imposed by these two constraints greatly facilitates fits of regression curves to any set of $\left(s^{2}, \bar{x}\right)$ pairs. Tokeshi's presumption of $s^{2} \geq \bar{x}$ is misleading inasmuch as values less than $\bar{x}$ are quite feasible even if on average $s^{2}>$ $\bar{x}$, but his basic premise is correct. Moreover, $s^{2}$ and $\bar{x}$ are self-correlated because $\bar{x}$ appears in the computational expression for $s^{2}$. Therefore, one might expect strong correlations between $s^{2}$ and $\bar{x}$ even when there is little or no relationship between $\sigma^{2}$ and $\mu$.

\section{The Mathematical Artifices}

\section{Constraints on Sample Variance}

Consider $n$ non-negative numbers $\left\{x_{1}, x_{2}, \ldots, x_{n}\right\}$ with mean $\bar{x}$. The maximum possible $s^{2}$ for a given $\bar{x}$ is achieved when one number equals $n \bar{x}$ and the rest are zero, i.e.,

$$
s_{\text {MAX }}^{2}=\frac{(n \bar{x}-\bar{x})^{2}+(n-1)(0-\bar{x})^{2}}{n-1}=n \bar{x}^{2}
$$




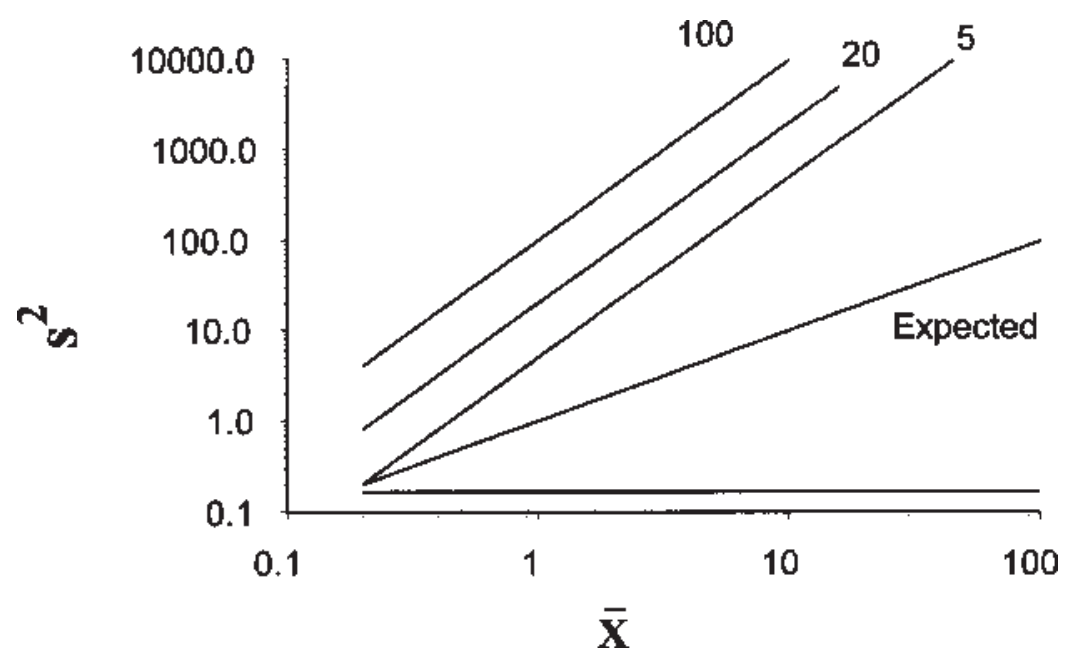

Figure 1. The feasible region of variance-mean regressions for sample sizes of 5, 20, and 100 in log-space. The bottom line is the average value of $s_{\text {MAX }}^{2}(1 / 6)$ and the line labeled "Expected" is the expected value of $s^{2}$ given $\bar{x}$ for sampling units (trips) with the same odds of catching fish (equation 3).

The minimum $s^{2}$ is zero when all the numbers have the same value, but for discrete variates this is only possible when $\bar{x}$ is an integer. Otherwise the minimum $s^{2}$ is

$$
s_{M I N}^{2}=(\bar{x}-i-1)(i-\bar{x}) \frac{n}{n-1}
$$

where $i$ is the integer part of $\bar{x}$ (see the Appendix). Notice that neither equation (1) nor (2) depend at all on the population parameters $\sigma^{2}$ and $\mu$. Thus, if $\sigma^{2}>s_{M A X}^{2}$, then the value of $s^{2}$ from that particular sample must underestimate $\sigma^{2}$. Likewise, if $\sigma^{2}<s_{\text {MIN }}^{2}$, then $s^{2}$ must overestimate $\sigma^{2}$.

The expected relationship of $s^{2}$ to $\bar{x}$ depends on the underlying pattern of the population, but lies somewhere between $s_{M I N}^{2}$ and $s_{M A X}^{2}$ (Fig. 1). Suppose $n$ trips combined to catch $T(=n \bar{x})$ fish and that each trip had the same (constant) ability to catch fish. Then the number caught per trip ought to be binomial distributed with probability $p=1 / n$ that a given fish will be assigned to a given trip. The expected variance of the binomial distribution given $T$ is $T p(1-p)$. Substituting $1 / n$ for $p$ we obtain the expected variance given $\bar{x}$ : 


$$
E\left[s^{2} \mid \bar{x}, n\right]=\frac{n-1}{n} \bar{x}
$$

Of course different fishing units can have different abilities to catch fish, in which case $E\left[s^{2} \mid \bar{x}, n\right]$ can exceed equation (3). In general however, if $\sigma^{2}$ $>E\left[s^{2} \mid \bar{x}, n\right]$, then the value of $s^{2}$ for that particular sample will probably (but not necessarily) underestimate $\sigma^{2}$.

In practice, the $s^{2}$ of continuous variates may take on any value between $s_{M I N}^{2}$ and $s_{M A X}^{2}$, some values being more likely than others depending on the underlying pattern of the data. The $s^{2}$ of discrete variates, however, can have only a finite number of values for a given $\bar{x}$ and $n$. Consider again the case where $n$ fishing trips were sampled from a population of fishing trips that are equally able to catch fish. The probability of obtaining a particular distribution of catch per trip $\left\{x_{1}, x_{2}, \ldots, x_{n}\right\}$ in any order is

$$
P\left(x_{1}, x_{2}, \ldots, x_{n}\right)=\frac{T !}{x_{1} ! x_{2} ! \ldots x_{n} !} \frac{n !}{n_{0} ! n_{1} ! \ldots n_{T} !} N^{-T}
$$

where $n_{x}$ is the number of variates with value $x$ and $T=\sum x_{i}$ (see pages 3840 in Feller 1968). The probability distribution (pdf) of $s^{2}$ is obtained from equation (4) by stepping through all possible distributions and adding the probabilities for those with the same variances. As Fig. 2 demonstrates, the pdf described by equation (4) is dominated by fine structure when $n$ is small. Some of the possible values of $s^{2}$ will rarely be observed despite the fact that both smaller and larger values may be common because there happen to be very few combinations that lead to them (a statistical artifact common to ratios of discrete quantities, see Johnston et al. 1995). The pdf becomes smoother and more values of $s^{2}$ become possible as $n$ increases, but the odds of $s^{2}$ being very much greater than its expected value, $\bar{x}(n-1) / n$, remain small. Thus, if $\sigma^{2}>>\bar{x}(n-1) / n$, then $s^{2}$ is likely to underestimate $\sigma^{2}$ even when $n$ is large. As $\bar{x}$ increases, however, the pdf of $s^{2}$ becomes less skewed, making it more likely that $s^{2}$ will attain a value near $\sigma^{2}$ and therefore less likely that $s^{2}$ will underestimate $\sigma^{2}$. Of course the pdf in Fig. 2 reflects the fact that the catching power of each trip was the same. Had the catching power differed, the expectation of $s^{2}$ would increase and the spread of the pdf would be different.

\section{Self-Correlation between Sample Variance and Sample Mean}

As mentioned earlier, $s^{2}$ and $\bar{x}$ are self-correlated because $\bar{x}$ appears in the formula for $s^{2}$. Kenney (1982) develops a general expression for the expected self-correlation between two variables, which for $s^{2}$ and $\bar{x}$ reduces to 


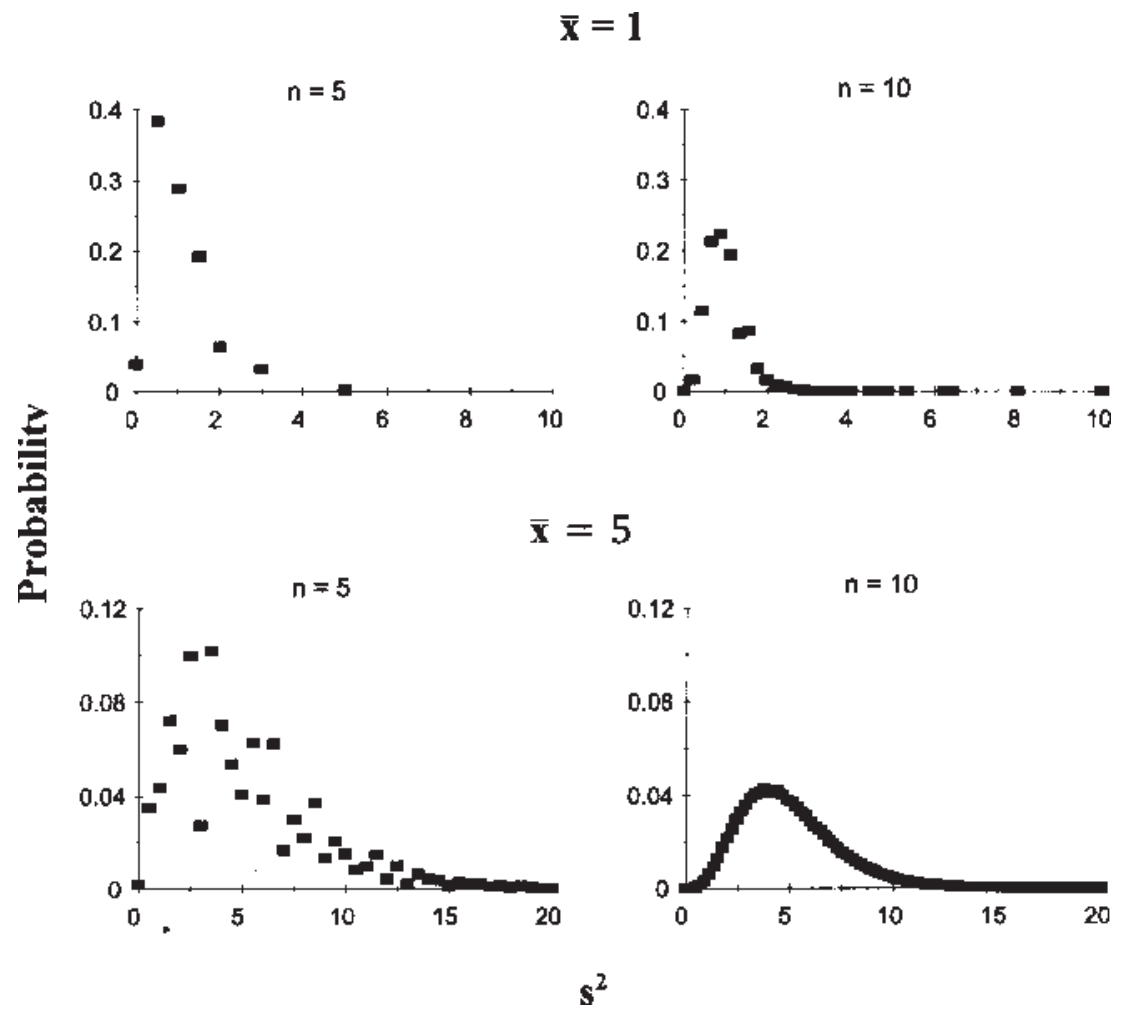

Figure 2. The pdf of the variances of discrete variates when the sampling units (trips) have the same odds of catching fish.

$$
r_{s^{2}, \bar{x}}=\frac{r_{p \bar{x}}\left(\sigma_{p} / \sigma_{q}\right)-r_{q \bar{x}}}{\sqrt{1+\left(\sigma_{p} / \sigma_{q}\right)^{2}-2 r_{p q}\left(\sigma_{p} / \sigma_{q}\right)}},
$$

where $s^{2}=p-q, p=\sum x^{2} /(n-1)$ and $q=n \bar{x}^{2} /(n-1)$. The correlation between $q$ and $\bar{x}$ is always close to 1 for non-negative variates. If it were possible for $p$ and $\bar{x}$ to be uncorrelated $\left(r_{p \bar{x}}=0\right)$, then also $r_{p q}=0$ and equation (5) would reduce to

$$
r_{s^{2}, \bar{x}}=\frac{-r_{q \bar{x}}}{\sqrt{1+\left(\sigma_{p} / \sigma_{q}\right)^{2}}} \approx \frac{-1}{\sqrt{1+\left(\sigma_{p} / \sigma_{q}\right)^{2}}}
$$


Table 2. Linear correlations of $s^{2}$ and $p=\sum x^{2} /(n-1)$ with $\bar{x}$ for selected probability densities and sample sizes (computed from 2,000 replicate samples drawn at random with replacement).

\begin{tabular}{lccccc}
\hline \multirow{2}{*}{$\begin{array}{l}\text { Density } \\
\left(\mu, \sigma^{2}\right)\end{array}$} & Statistic & \multicolumn{4}{c}{ Sample size $(n)$} \\
\cline { 3 - 6 } Lognormal & $p$ & 0.87 & 0.82 & 0.80 & 0.79 \\
$(5,25)$ & $s^{2}$ & 0.74 & 0.72 & 0.67 & 0.65 \\
Lognormal & $p$ & 0.99 & 0.99 & 0.99 & 0.99 \\
$(100,500)$ & $s^{2}$ & 0.35 & 0.39 & 0.38 & 0.43 \\
Neg. bin. & $p$ & 0.90 & 0.89 & 0.89 & 0.90 \\
(5, 25) & $s^{2}$ & 0.62 & 0.68 & 0.66 & 0.70 \\
Neg. bin. & $p$ & 0.99 & 0.99 & 0.99 & 0.99 \\
$(100,500)$ & $s^{2}$ & 0.17 & 0.25 & 0.24 & 0.27 \\
Poisson & $p$ & 0.97 & 0.96 & 0.96 & 0.96 \\
(5, 5) & $s^{2}$ & 0.21 & 0.25 & 0.30 & 0.29 \\
Poisson & $p$ & 1.00 & 1.00 & 1.00 & 1.00 \\
$(100,100)$ & $s^{2}$ & 0.01 & 0.02 & 0.04 & 0.04 \\
\hline
\end{tabular}

This implies that including $\bar{x}$ on both sides of the equation imparts a strong negative correlation unless $\sigma_{p} \gg \sigma_{q}$ (which depends on the skewness of the distribution of $x$ ).

\section{Some Numerical Illustrations}

This section uses simulated data to illustrate how the mathematical artifices discussed above can influence one's perception of the variance to mean relationship.

\section{Example 1: Unchanging Population ( $\mu$ and $\sigma^{2}$ Constant)}

Six data sets of 2,000 $\left(s^{2}, \bar{x}\right)$ pairs each were simulated by randomly drawing $n$ observations with replacement from three types of statistical distributions (Poisson, negative binomial and lognormal) and two parameter sets ( $\mu=5, \sigma^{2}=25$ and $\mu=100, \sigma^{2}=500$ ). The linear correlation between $p=\sum x^{2} /(n-1)$ and $\bar{x}$ was 0.8 or larger in all six cases, but the correlation between $s^{2}$ and $\bar{x}$ depended on the distribution (Table 2, Fig. 3). Sample size ( $n$ ) was relatively unimportant; however, the magnitudes of $\mu$ and $\sigma^{2}$ were very important. Samples drawn from the distributions with $\mu=5$ had much higher $\left(s^{2}, \bar{x}\right)$ correlations than those drawn from the distributions with $\mu=100$. Likewise, the samples drawn from distributions with high $\sigma^{2}$ 

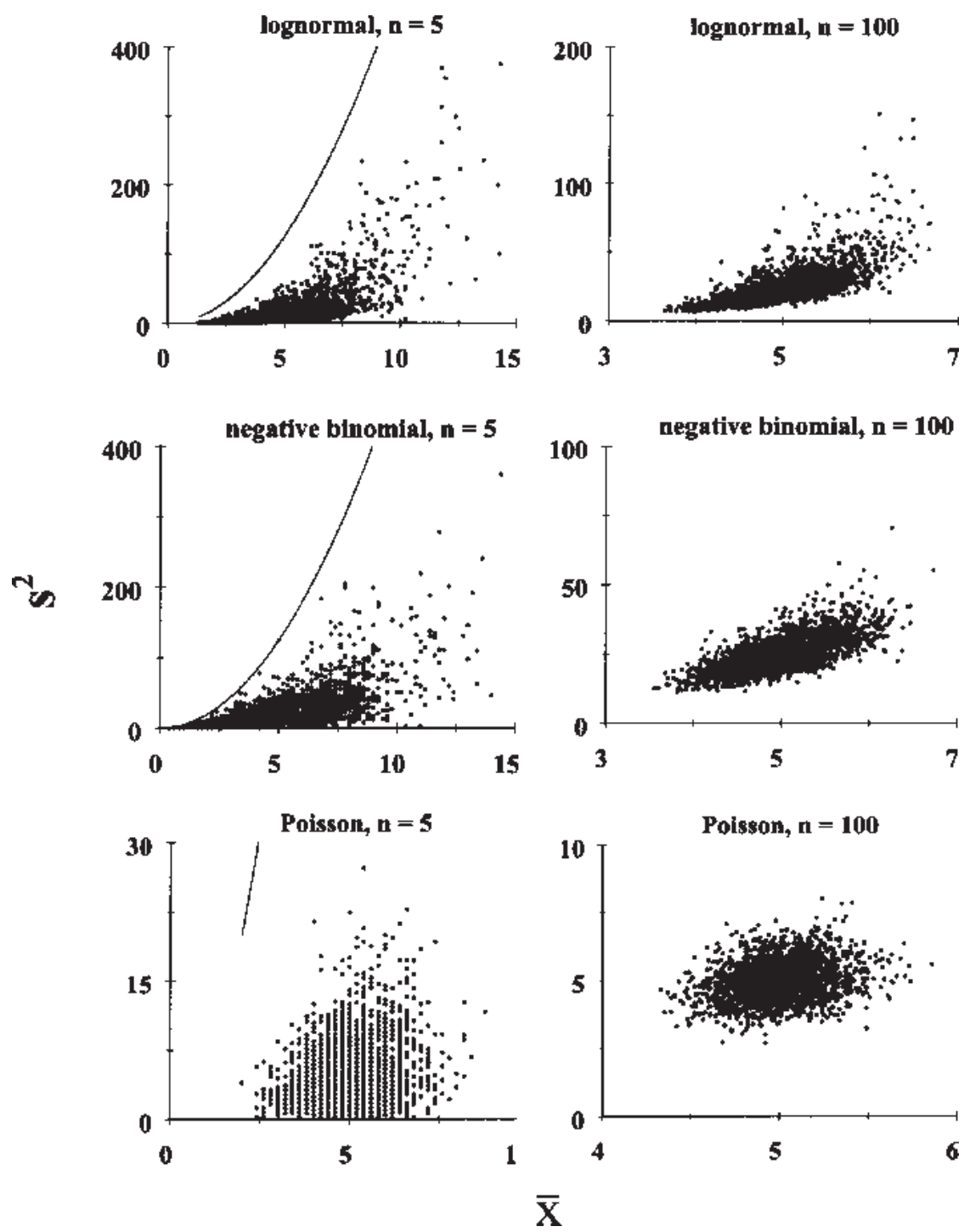

Figure 3. Scatter plots of $\left(s^{2}, \bar{x}\right)$ pairs from samples of 5 or 100 drawn from the lognormal and negative binomial densities with mean 5 and variance 25 and the Poisson density with mean 5 and variance 5 . The adjacent curves represent $s_{M A X}^{2}$. 
(lognormal and negative binomial) had much higher $\left(s^{2}, \bar{x}\right)$ correlations than those drawn from the Poisson distribution (with $\sigma^{2}=\mu$ ). Not surprisingly, the power function $\left(\log _{e} s^{2}=\log _{e} a \bar{x}^{b}\right)$ fit the data generated from the lognormal and negative binomial distributions with $\mu=5$ and $\sigma^{2}=25$ quite well, yielding coefficients of determination $\left(r^{2}\right)$ in excess of 0.5 , but provided a very poor fit to the data generated from the remaining distributions.

\section{Example 2: Changing $\mu$, but Constant $\sigma^{2}$}

Now consider a situation where samples are drawn from a stock that has changed substantially over time, but for which the variance remains constant. Data sets consisting of $20\left(s^{2}, \bar{x}\right)$ pairs were constructed by randomly choosing 20 values $\left(Y_{j}\right)$ between 0 and 10 and then sampling $n$ times with replacement from lognormal distributions with $\sigma^{2}=25$ and $\mu=Y_{j}$. The 20 sample $\left(s^{2}, \bar{x}\right)$ pairs in each simulated data set were then fitted with the power function. The average $r^{2}$ from 1,000 such data sets were surprisingly high $(0.59,0.78,0.75$ and 0.69 for $n=2,5,10$, and 20). The values of $s^{2}$ generally fluctuated around $\sigma^{2}(25)$ when $\bar{x}$ was large, but severely underestimated $\sigma^{2}$ when $\bar{x}$ was small (Fig. 4 ).

\section{Example 3: All Possible Sample Variances Equally Likely}

In this example $s^{2}$ was allowed to assume any value between the minimum and maximum feasible values (equations 1 and 2 ) with equal probability. This is equivalent to a situation where the underlying pattern of a continuous variate is completely inconsistent. Artificial data sets were generated by randomly selecting $20 \bar{x}$ values and assigning to each $\bar{x}$ a corresponding $s^{2}$ drawn at random from the feasible region. The power function was then fitted to each data set by least squares after taking the logarithms of $s^{2}$ and $\bar{x}$. The median $r^{2}$ was about 0.8 and values under 0.5 were observed for only $10 \%$ of the simulated data sets. Sample size had little impact on the $r^{2}$ because the possible variances were uniformly distributed across the sampling space and the sampling space for $n=5$ is similar to that for $n=100$ on a logarithmic scale (Fig. 1). Thus, it is clear that the restricted feasible region for $\left(s^{2}, \bar{x}\right)$ by itself can account for a large fraction of the strong correlations between the two statistics.

\section{Discussion}

Although $s^{2}$ is an unbiased estimator of $\sigma^{2}$, the value of $s^{2}$ conditional on $\bar{x}$ is not. The statistics $s^{2}$ and $\bar{x}$ are spuriously correlated because $\bar{x}$ appears in the computation of $s^{2}$ and because the probability space of $s^{2}$ is restricted, especially when $n$ or $\bar{x}$ are small. As the examples show, it is possible to find a strong correlation between $s^{2}$ and $\bar{x}$ even when $\sigma^{2}$ and 


$$
n=2
$$

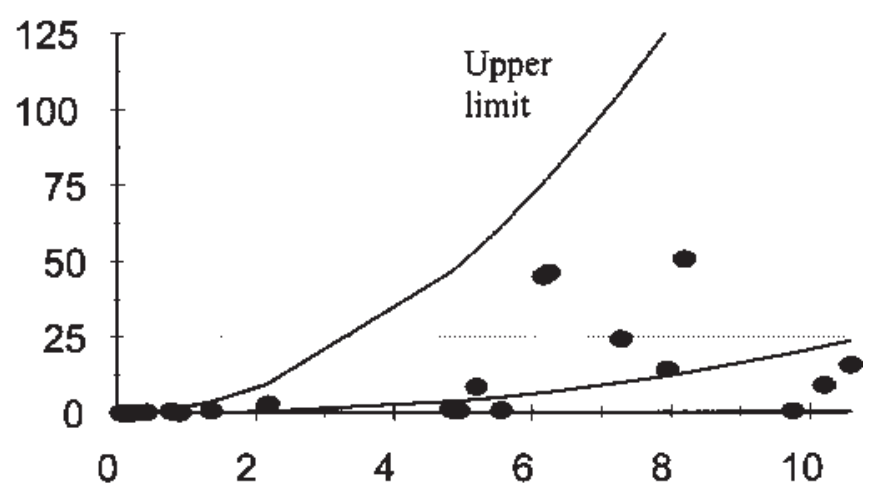

ro

$$
n=20
$$

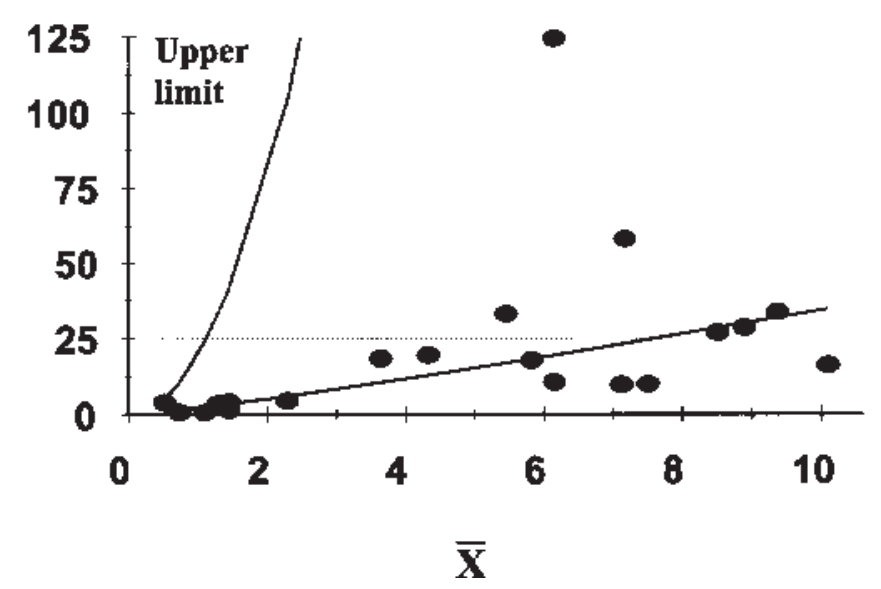

Figure 4. Typical fits of the power function to the simulated data (dots) in example 2. The dashed line indicates the true variance (25). 
$\mu$ are unrelated. Similarly, where a relationship between $\sigma^{2}$ and $\mu$ does exist, regressions of $s^{2}$ and $\bar{x}$ may not accurately reflect it.

Other factors may further confound the $s^{2}$ to $\bar{x}$ relationship. Downing (1986) showed empirically that estimates of the exponent parameter of the power function vary systematically with the number of $\left(s^{2}, \bar{x}\right)$ pairs used as well as $n$. Additional bias may occur if $n$ varies among the $\left(s^{2}, \bar{x}\right)$ pairs since the pdf of $s^{2}$ varies with $n$. The method used to fit the data can also bias the results. Ross (1990) pointed out that the logarithm of $s^{2}$ tends to be biased low, particularly for highly skewed data with high $\bar{x}$ and low $n(<30)$. This tends to cause the curvature parameters of the regression equation to be underestimated (Clark and Perry 1994). On the other hand, failing to use logarithms typically reduces the precision of the estimates because the variance of $s^{2}$ tends to increase with $\bar{x}$ and because anomalous points are allowed excessive leverage (Taylor et al. 1978).

Still another problem arises when the maximum value the variate can attain is finite. The number of fish kept per trip, for example, might be limited to $L$ owing to regulations or gear saturation. In such cases the maximum possible $s^{2}$ for a given $\bar{x}$ is

$$
s_{M A X}^{2}=\frac{i(L-\bar{x})^{2}+(n-i-1) \bar{X}^{2}+(R-\bar{x})^{2}}{n-1},
$$

where $i$ is the integer part of $n \bar{x} / L$ and $R$ is the remainder. Clearly $s_{M A X}^{2}$ decreases to zero as $\bar{x}$ approaches $L$, therefore the $s^{2}$ to $\bar{x}$ relationship will exhibit a dome-shaped trend regardless of the statistical distribution of the population or the relationship between $\sigma^{2}$ and $\mu$. For example, if all of the observations happen to be either 0 or 1 , then $s^{2}$ always equals $n\left(\bar{x}-\bar{x}^{2}\right) /(n-1)$ and there is absolutely no possibility of deducing the relationship between $\sigma^{2}$ and $\mu$. One could attempt to derive a suitable transformation from the fit of a quadratic equation to the $\left(s^{2}, \bar{x}\right)$ pairs, but this would be subject to the same caveats discussed above.

In summary, it is clear that the $s^{2}$ and $\bar{x}$ of non-negative variates are spuriously correlated. The examples suggest that, when $\mu$ is small and $\sigma^{2}$ is large, $s^{2}$ tends to underestimate $\sigma^{2}$ in some proportion to the degree that $\bar{x}$ underestimates $\mu$. Inasmuch as uncommon resources with high variance are frequently encountered in fisheries work, this statistical artifice is likely to pose a significant challenge. In general, weighting estimates from scarce resources by $s^{2} / n$ may bias the assessment by overemphasizing estimates that happen to be too low. This is especially likely to be a problem when $n$ is small because $\bar{x}$ is more likely to be poorly estimated and improperly weighted; therefore it may be prudent to simply ignore statistics from small samples. It must be emphasized, however, that the strong spurious correlation between $s^{2}$ and $\bar{x}$ persists even with large $n$ and that regressions of $s^{2}$ and $\bar{x}$ may be highly significant even when there is no relationship between $\sigma^{2}$ and $\mu$ as long as some of the $\bar{x}$ values 
used in the regression are small. Accordingly, weighting schemes or transformations that are based on the empirical relationship between $s^{2}$ and $\bar{x}$ are apt to be meaningless unless $\mu$ is large $(>>5)$ or $\sigma^{2}$ is small (on the order of $\mu$ ). Perhaps a better alternative might be to employ transformations or objective functions that are known to be robust to the type of deviations one is likely to observe in fisheries data (e.g., Otter Research Ltd. 1994, Schnute and Richards 1995), but more work is needed.

\section{Acknowledgments}

I thank F. Funk, V. Restrepo, D. Vaughan, J. Zweifel, and two anonymous reviewers for their helpful comments.

\section{References}

Bartlett, M.S. 1947. The use of transformations. Biometrics 3:39-52.

Clark, S.J., and J.N. Perry. 1994. Small sample estimation for Taylor's power law. Environ. Ecol. Stat. 1:287-302.

Curtis, J.H. 1943. On transformations used in analysis of variance. Ann. Math. Stat. 14:10.

Cyr, H., J.A. Downing, S. Lalonde, S.B. Baines, and M.L. Pace. 1992. Sampling larval fish populations: Choice of sample number and size. Trans. Am. Fish. Soc. 121:356-368.

Deriso, R.B., and A.M. Parma. 1987. On the odds of catching fish with angling gear. Trans. Am. Fish. Soc. 116:244-256.

Dong, Q., and V.R. Restrepo. 1996. Notes on the Poisson error assumption made to estimate relative abundance of West Atlantic bluefin tuna. Int. Comm. Conserv. Atl. Tunas, Coll. Vol. Sci. Pap. 45(2):158-161.

Downing, J.A. 1986. Spatial heterogeneity: Evolved behavior or mathematical artefact. Nature 323:255-257.

Downing, J.A. 1989. Precision of the mean and the design of benthos sampling programmes: Caution revised. Mar. Biol. 103:231-234.

Feller, W. 1968. An introduction to probability theory and its applications. Volume 1. John Wiley and Sons, New York. 509 pp.

Green, R.H. 1979. Sampling design and statistical methods for environmental biologists. John Wiley and Sons, New York. 257 pp.

Houser, A., and J.E. Dunn. 1967. Estimating the size of the threadfin shad population in Bull Shoals Reservoir from midwater trawl catches. Trans. Am. Fish. Soc. 96:176-184.

Johnston, R.G., S.D. Schroder, and R.A. Mallawaaratchy. 1995. Statistical artifacts in the ratio of discrete quantities. The American Statistician 49(3):285-291.

Kenney, B.C. 1982. Beware of spurious self-correlations! Water Resour. Res. 18(4):1041-1048. 
Lester, N.P., M.M. Petzold, and W.I. Dunlop. 1991. Sample size determination in roving creel surveys. Amer. Fish. Soc. Symp. 12:25-39.

Mangel, M., and J.H. Beder. 1985. Search and stock depletion: Theory and applications. Can. J. Fish. Aquat. Sci. 42:150-163.

Myers, R.A., and P. Pepin. 1991. Reply to: On testing the robustness of lognormalbased estimators. Biometrics 47:1623-1624.

Otter Research Ltd. 1994. AUTODIF: A C++ array language extension with automatic differentiation for use in nonlinear modeling and statistics. Otter Research Ltd., Nanaimo, B.C.

Pella, J.J., and C.T. Psaropulos. 1975. Measures of tuna abundance from purseseine operations in the eastern Pacific Ocean adjusted for fleet-wide evolution of increased fishing power, 1960-1971. Inter-Am. Trop. Tuna Comm. Bull. 16:283-354.

Pennington, M. 1996. Estimating the mean and variance from highly skewed marine data. Fish. Bull. U.S. 94:498-505.

Porch, C.E., and W.W. Fox. 1990. Simulating the dynamic trends of fisheries regulated by small daily bag limits. Trans. Am. Fish. Soc. 119:836-849.

Ross, G.J.S. 1990. Incomplete variance functions. Journal of Applied Statistics 17:3-8.

Schnute, J.T, and L.J. Richards. 1995. The influence of error on population estimates from catch-age models. Can. J. Fish. Aquat. Sci. 52:2063-2077.

Small, I., and D.Y. Downham. 1985. The interpretation of angler's records (trout and seatrout, Salmo trutta L., and Salmo salar L.) Aquacult. Fish. Manage. 16:151169.

Smith, S.J.1990. Use of statistical models for the estimation of abundance from groundfish trawl survey data. Can. J. Fish. Aquat. Sci. 47:894-903.

Taylor, C.C. 1953. Nature of variability in trawl catches. Fish. Bull. U.S. 54:145-166.

Taylor, L.R. 1980. New light on the variance/mean view of aggregation and transformation: Comment. Can. J. Fish. Aquat. Sci. 37:1330-1332.

Taylor, L.R., and I.P. Woiwod. 1982. Comparative synoptic dynamics. I. Relationships between inter- and intra-specific spatial and temporal variance/mean population parameters. J. Anim. Ecol. 51:879-906.

Taylor, L.R., I.P. Woiwod, and J.N. Perry. 1978. The density-dependence of spatial behavior and the rarity of randomness. J. Anim. Ecol. 47:383-406.

Taylor, L.R., R.A. Taylor, I.P. Woiwod, and J.N. Perry. 1983. Behavioural dynamics. Nature 303:801-804.

Tokeshi, M. 1995. On the mathematical basis of the variance-mean power relationship. Resource Population Ecology 37(1):43-48.

Warwick, R.M., and K.R. Clarke. 1993. Increased variability as a symptom of stress in marine communities. J. Exp. Mar. Biol. Ecol. 172:215-225.

Welch, D.W., and Y. Ishida. 1993. On the statistical distribution of salmon in the sea: Application of the negative binomial distribution, and the influence of sampling effort. Can. J. Fish. Aquat. Sci. 50:1029-1038. 


\section{Appendix: Derivation of the Minimum Possible Variance}

Taylor and Woiwood (1982) show that, if the total number of objects $T$ is less than $n$, then $s_{M I N}^{2}=n\left(\bar{x}-\bar{x}^{2}\right) /(n-1)$. This expression can be generalized to any value of $T$ by noting that the minimum variance is obtained when the number of objects is distributed as evenly as possible among the two integer categories $i$ and $i+1$, where $i$ is the integer part of $\bar{x}$. Designating the number of sampling units containing $i$ objects as $n_{i}$, we have

$$
\begin{aligned}
& T=(i+1) n_{i+1}+i n_{i} \\
& n=n_{i+1}+n_{i} .
\end{aligned}
$$

Solving for $n_{i+1}$ and $n_{i}$ and then substituting them into the expression for sample variance yields

$$
s_{M I N}^{2}=\frac{(T-i n)(i+1-\bar{x})^{2}+[(i+1) n-T](i-\bar{x})^{2}}{n-1}
$$

which simplifies to

$$
s_{M I N}^{2}=(\bar{x}-i-1)(i-\bar{x}) \frac{n}{n-1} .
$$




\title{
Diagnosing Systematic Errors in Reported Fishery Catch
}

\author{
Stratis Gavaris and Lutgarde Van Eeckhaute \\ Department of Fisheries and Oceans, Biological Station, St. Andrews, N.B., \\ Canada
}

\begin{abstract}
Age structured assessment methods integrate information from the commercial fishery catch and from indices of abundance. Systematic errors in the catch at age, often caused by mis-reporting of landings or discarding of small fish, could introduce appreciable bias to the results of stock assessments. Important inconsistencies were revealed when results from a base assessment employing catch at age were compared to results from a model that employs proportion caught at age but estimates total numbers caught each year in addition to the other model parameters, terminal year abundance and index catchability. For eastern Georges Bank haddock, the diagnostic model suggested that the base model had underestimated the year-class abundance for years prior to 1977. It also indicated that the mid-1980s year classes might have been overestimated by the base model. Though not recommended for estimation, the diagnostic model subjects data to different assumptions, making it useful as an investigative tool for evaluating the robustness of results.
\end{abstract}

\section{Introduction}

Fish stock assessment methods for age-structured analyses integrate auxiliary information from population abundance trend indices with data from catch removals by the fisheries to estimate stock status (e.g., Fournier and Archibald 1982, Deriso et al. 1985, Gavaris 1988). Deviations between observed and predicted quantities for these models are assumed to be random. Abundance indices are often derived from controlled research survey data or from a selected subset of fishery catch and effort records. Therefore, appropriate measures, such as random selection of survey locations, maintaining consistent fishing practices on surveys from year to year or factoring out gear, seasonal, and spatial effects from catch rates, can be taken to prevent the introduction of systematic deviations. Commercial 
fishery catch data, however, may be suspect of containing errors which display a non-random pattern. Fishery catch removals by age can be obtained by applying the age composition derived from samples for length composition and aging material to the total number caught (Gavaris and Gavaris 1983). The total amount caught is typically based on a complete census of fishery landings and ancillary information on discards, if available. Systematic errors in the catch data are commonly due to mis-reporting of total amount caught, although the samples for length composition may also be biased if size-selective culling has occurred prior to observation.

Systematic errors in the catch-at-age could introduce appreciable bias to the results of stock assessments. A constant mis-reporting rate may not pose problems but trends in the total amount or the age composition of mis-reported catch result in complex patterns of bias (Sinclair et al. 1991). Employing assessment models that accommodate random error in the catch-at-age would not be an appropriate treatment for this problem. Concern regarding the reliability of catch data has stimulated development of methods that employ only survey data. One approach proposed by Cook assumes separable age and year effects on fishing mortality rate (ICES 1997) while Sinclair and Chouinard (1992) employed a multiplicative model and assumed a constant fishing mortality rate over some period. Patterson retained the assumption of separable fishing mortality rate and extended the method to include sampling data from the commercial fishery (ICES 1997).

Many assessments are conducted without invoking the separable fishing mortality rate assumption because year-to-year variation in the exploitation pattern by age has been observed and is considered meaningful. We compare results from a non-separable assessment employing catch-atage and abundance indices to those from a non-separable model that did not use total number caught but employed proportion caught-at-age and abundance indices. The total number caught each year was estimated in addition to the other model parameters. We will refer to the latter as the diagnostic model and the former as the base model. These models were applied to data for eastern Georges Bank haddock. We do not promote the diagnostic model for estimation purposes. The purpose of this study was to explore the utility of the diagnostic model, which places less reliance on suspect fishery catch data, as a tool for investigating inconsistencies which might be caused by systematic errors.

\section{Methods}

The commercial fishery catch-at-age is used to reconstruct the past using virtual population analysis (VPA). Due to the error propagation properties of VPA (Pope 1972, Sampson 1988), random errors in the catch-at-age typically have negligible impact on assessment calibration methods compared to random errors in the abundance indices (Sinclair et al. 1991). Capitaliz- 
ing on this characteristic can greatly reduce the number of unknown parameters. It is not uncommon, therefore, to encounter assessments which ignore the random error in catch-at-age. Both the base and diagnostic models adopt this tact. In its general form, the VPA calculations require an estimate of population abundance for each year class. With suitable assumptions about fishing mortality rate patterns on older ages, the calculations can be done if we have estimates of population abundance for the terminal year of the analysis. The abundance indices are the auxiliary data used to obtain those estimates by a process referred to as calibration of the VPA. The calibration process involves fitting abundance index data and population abundance results from VPA to a catchability model to estimate the parameters of that relationship, referred to as the calibration constants. A search is conducted to find the terminal year-class abundance estimates for the VPA that result in the best fit to the catchability model.

For eastern Georges Bank haddock, the sampling data from the commercial fishery were used to derive estimates of annual proportion caught at age, $P_{a, t}$, for ages $a=0,1,2, \ldots, 8$ and periods $t=1969,1970,1971, \ldots$, 1996. The time period label signifies the beginning of the period during which the catch occurred. The commercial fishery catch information may be used with the samples to derive estimates of annual numbers caught at age, $C_{a, t}$, for the same ages and periods. The procedures described in Gavaris and Gavaris (1983) were used to combine samples and to apply the catch information. The available abundance indices, $I_{s, a, t}$, were from bottom trawl research surveys conducted by the Canadian Department of Fisheries and Oceans (DFO) and the U.S. National Marine Fisheries Service (NMFS):

for $s=$ DFO spring survey, ages $a=1,2, \ldots, 8$, times $t=1986.16$, $1987.16, \ldots, 1997.16$

$s=$ NMFS spring survey, ages $a=1,2, \ldots, 8$, times $t=1969.29,1970.29, \ldots$, 1996.29

$s=$ NMFS fall survey, ages $a=0,1, \ldots, 5$, times $t=1969.69,1970.69, \ldots$, 1996.69.

The fall survey captures young of the year and that information is included as 0 group, but older haddock appear less available during this season. Survey indices were not included for older ages where catches were sparse and there were frequent occurrences of zero catches. Accordingly, a plus group was not included in the VPA as it would have no impact on the calibration. If desired, the abundance of the plus group in the population could be computed by making a suitable assumption about its fishing mortality rate and applying the catch equation (equation 3 below). Zero observations for abundance indices were treated as missing data. Further specific details of the data used for the assessment are provided in Gavaris and Van Eeckhaute (1997). 
The model formulation employed for the base model assumed that the random error in the catch at age was negligible and the annual total number caught, $L_{t}$, were reported accurately. The catch at age then can be expressed as $C_{a, t}=L_{t} P_{a, t}$. The errors in the abundance indices were assumed independent and identically distributed after taking natural logarithms of the values. The annual natural mortality rate, $M$, was assumed constant and equal to 0.2. A model formulation, using as parameters the natural logarithm of population abundance at the beginning of the year, was considered because of close to linear behavior for such a parameterization (Gavaris 1993). The following model parameters were defined: $\theta_{a, t^{\prime}}=\ln$ population abundance for ages $a=1,2, \ldots, 8$ at time $t^{\prime}=1997.25 ; \kappa_{s, a}=\ln$ calibration constants for each abundance index source $s$ and relevant ages $a$. We solved for the parameters by minimizing the sum of squared differences between the natural logarithm observed abundance indices and the natural logarithm population abundance adjusted for catchability by the calibration constants. The objective function for minimization was defined as

$$
\underset{s, a, t}{\Psi}(\hat{\theta}, \hat{\kappa})=\sum_{s, a, t}\left[\psi_{s, a, t}(\hat{\theta}, \hat{\kappa})\right]^{2}=\sum_{s, a, t}\left\{\ln I_{s, a, t}-\left[\hat{\kappa}_{s, a}+\ln N_{a, t}(\hat{\theta})\right]\right\}^{2}
$$

For convenience, the population abundance $N_{a, t}(\hat{\theta})$ is abbreviated by $N_{a, t^{*}}$ At time $t^{\prime}$, the population abundance was obtained directly from the parameter estimates, $N_{a, t^{\prime}}=e^{\hat{\theta}_{a, t^{\prime}}}$. For all other times, the population abundance was computed using the virtual population analysis algorithm, which incorporates the common exponential decay model (Beverton and Holt 1957)

$$
N_{a+\Delta t, t+\Delta t}=N_{a, t} e^{-\left(F_{a, t}+M_{a}\right) \Delta t}
$$

Year was used as the unit of time; therefore ages were expressed as years and the fishing and natural mortality rates were annual instantaneous rates. The fishing mortality rate $F_{a, t}$ exerted during the time interval $t$ to $t$ $+\Delta t$, was obtained by solving the catch equation

$$
C_{a, t}=\frac{F_{a, t} \Delta t N_{a, t}\left(1-e^{-\left(F_{a, t}+M_{a}\right) \Delta t}\right)}{\left(F_{a, t}+M_{a}\right) \Delta t}
$$

using a Newton-Raphson algorithm. The fishing mortality rate for the oldest age in the last time interval of each year was assumed equal to the weighted average for ages fully recruited to the fishery during that time interval 


$$
F_{8, t}=\frac{\sum_{a=4}^{7} N_{a, t} F_{a, t}}{\sum_{a=4}^{7} N_{a, t}}
$$

The diagnostic model was similar in every respect to the base model except that we rejected the assumption that the total number caught each year by the fishery was reported accurately. Therefore, in addition to the model parameters $\theta_{a, t^{\prime}}$ and $\kappa_{s, a}$, the total number caught each year were estimated, $\lambda_{t}=$ total number caught for time period $t=1969,1970, \ldots$, 1996. The catch-at-age was obtained from $C_{a, t}=\lambda_{t} p_{a, t}$ and the computations thereafter were the same as for the base model. To maintain comparability, all other assumptions were the same as those for the base model. Both the base model and the diagnostic model were implemented within ADAPT (Gavaris 1988), which employs a Marquardt nonlinear least squares algorithm.

With both models, the covariance matrix of the parameters was estimated using the common linear approximation (Kennedy and Gentle 1980, p. 476)

$$
\operatorname{Cov}(\hat{\theta}, \hat{\kappa})=\hat{\sigma}^{2}\left[J^{T}(\hat{\theta}, \hat{\kappa}) J(\hat{\theta}, \hat{\kappa})\right]^{-1}
$$

where $\hat{\sigma}^{2}$ is the mean square residual and $J(\hat{\theta}, \hat{\kappa})$ is the Jacobian matrix. The bias of the parameters was estimated using Box's (1971) approximation, which assumes that the errors are normally distributed

$$
\begin{aligned}
\operatorname{Bias}(\hat{\theta}, \hat{\kappa})= & \frac{-\hat{\sigma}^{2}}{2}\left[\sum_{s, a, t} J_{s, a, t}(\hat{\theta}, \hat{\kappa}) J_{s, a, t}^{T}(\hat{\theta}, \hat{\kappa})\right]^{-1}\left[\sum_{s, a, t} J_{s, a, t}(\hat{\theta}, \hat{\kappa})\right] t r \\
& \left\{\left[\sum_{s, a, t} J_{s, a, t}(\hat{\theta}, \hat{\kappa}) J_{s, a, t}^{T}(\hat{\theta}, \hat{\kappa})\right]^{-1} H_{s, a, t}(\hat{\theta}, \hat{\kappa})\right\}
\end{aligned}
$$

where $J_{s, a, t}(\hat{\theta}, \hat{\kappa})$ are vectors of the first derivatives for each $\psi_{s, a, t}(\hat{\theta}, \hat{\kappa})$ and $H_{s, a, t}(\hat{\theta}, \hat{\kappa})$ are the Hessian matrices for each $\psi_{s, a, t}(\hat{\theta}, \hat{\kappa})$.

Population quantities of interest for management advice are functions of the estimated parameters. Denote an arbitrary quantity by $\hat{\alpha}=g(\hat{\theta}, \hat{\kappa})$ where $g$ is the transformation function. The variance and bias were estimated using the methods described in Ratkowsky (1983)

$$
\operatorname{Var}(\hat{\alpha})=\operatorname{tr}\left[G G^{T} \operatorname{cov}(\hat{\theta}, \hat{\kappa})\right]
$$




$$
\operatorname{Bias}(\hat{\alpha})=G^{T} \operatorname{Bias}(\hat{\theta}, \hat{\kappa})+\frac{\operatorname{tr}[W \operatorname{cov}(\hat{\theta}, \hat{\kappa})]}{2}
$$

where $G$ is the vector of first derivatives of $g$ with respect to parameters and $W$ is the matrix of second derivatives of $g$ with respect to parameters.

\section{Results}

Proportion caught at age and abundance indices do not contain any information on the absolute magnitude of the population. Therefore, to make the diagnostic model determinate, it is necessary to set one of the parameters to a fixed value. We arbitrarily assigned the abundance of the 1992 year class at time $t^{\prime}=1997.25$. In retrospect, we suggest that it might be better to select one of the estimated catches to be assigned a fixed value instead of one of the year-class estimates as we did. This implementation modification would not alter the basic results but it would avoid unduly restricting a single year-class estimate, as we observed in the sensitivity trials.

The first attempt to estimate all the remaining parameters in the diagnostic model failed. The abundance indices for this resource, which were derived from bottom trawl surveys, were variable and occasionally indicated an increase in the abundance of a year class (Fig. 1) when mortality processes dictate a decline. In these instances, the model estimates of total numbers caught tend to zero, an unrealistic result. Seven of the 29 years displayed this behavior, so we assigned fixed values to these parameters equal to the reported total number caught in those years. We investigated the sensitivity of the results to these assumed values and report on that later.

Parameter estimates from nonlinear models are typically biased, although the bias may be negligible if the parameter behavior is close to linear. The estimates of population abundance needed to compute the VPA for eastern Georges Bank haddock have substantial bias and the VPA computed with bias adjusted estimates is used as the basis for resource evaluation (Gavaris and Van Eeckhaute 1997). Further, the nature of the bias for the base model and for the diagnostic model displayed different characteristics. Consequently, all comparisons were based on bias adjusted results.

Since the absolute magnitude of the population abundance was arbitrarily set by the fixed parameters, we compared patterns of relative yearclass abundance. The base and diagnostic models both identified the two largest year classes, 1975 and 1978, and the 1983, 1985, 1987 and 1992 year classes which were of intermediate magnitude (Fig. 2). The diagnostic model indicated that the 1971 and 1972 year classes were relatively stronger than the estimates obtained from the base model. In general, the rankings of year classes from the two models were comparable (rank cor- 


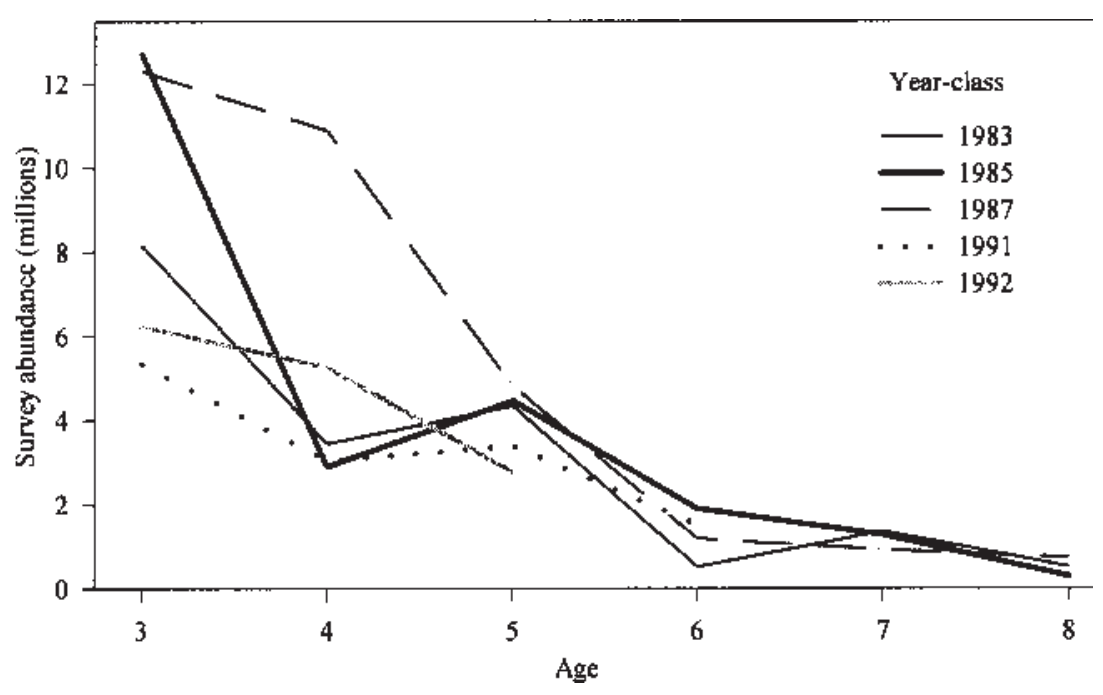

Figure 1. As year classes increase in age, mortality processes dictate a decline in abundance with each successive year. However, year-class abundance estimates from bottom trawl surveys for Georges Bank haddock at ages for which they are fully recruited to the survey sometimes show an increase in abundance due to sampling variability.

relation $=0.98$ ). Better resolution for comparison of the trends is obtained by comparing the ratio of diagnostic model estimates to the base model estimates. The ratio was typically about two but there was a tendency for somewhat higher values in the earlier years. The largest deviations occurred for the 1971 and 1972 year classes. The deviation for the large 1975 year class was among the larger ones at $50 \%$ greater than the modal value. There also appears to be a short period during the mid-1980s when the ratios were smaller. The relative errors for year-class abundance from the two models show markedly different patterns. The base model results, on the merit of assumed known catch-at-age, show a pattern of rapidly diminishing error for earlier year classes while the diagnostic model results remain constant at about $50 \%$ over the entire period (Fig. 2).

Comparison of the fishing mortality rates for ages 4 to 8 , which were considered to be fully recruited to the fishery, indicated that the results from the diagnostic model were much more erratic (Fig. 3). In the diagnostic model, the exploitation pattern is derived principally from the abundance indices, which, as has been noted, are quite variable. This type of erratic behavior is not uncharacteristic of mortality rates derived from abundance indices alone. Further, the fishing mortality pattern from the diagnostic model does not correspond well with that from the base model 


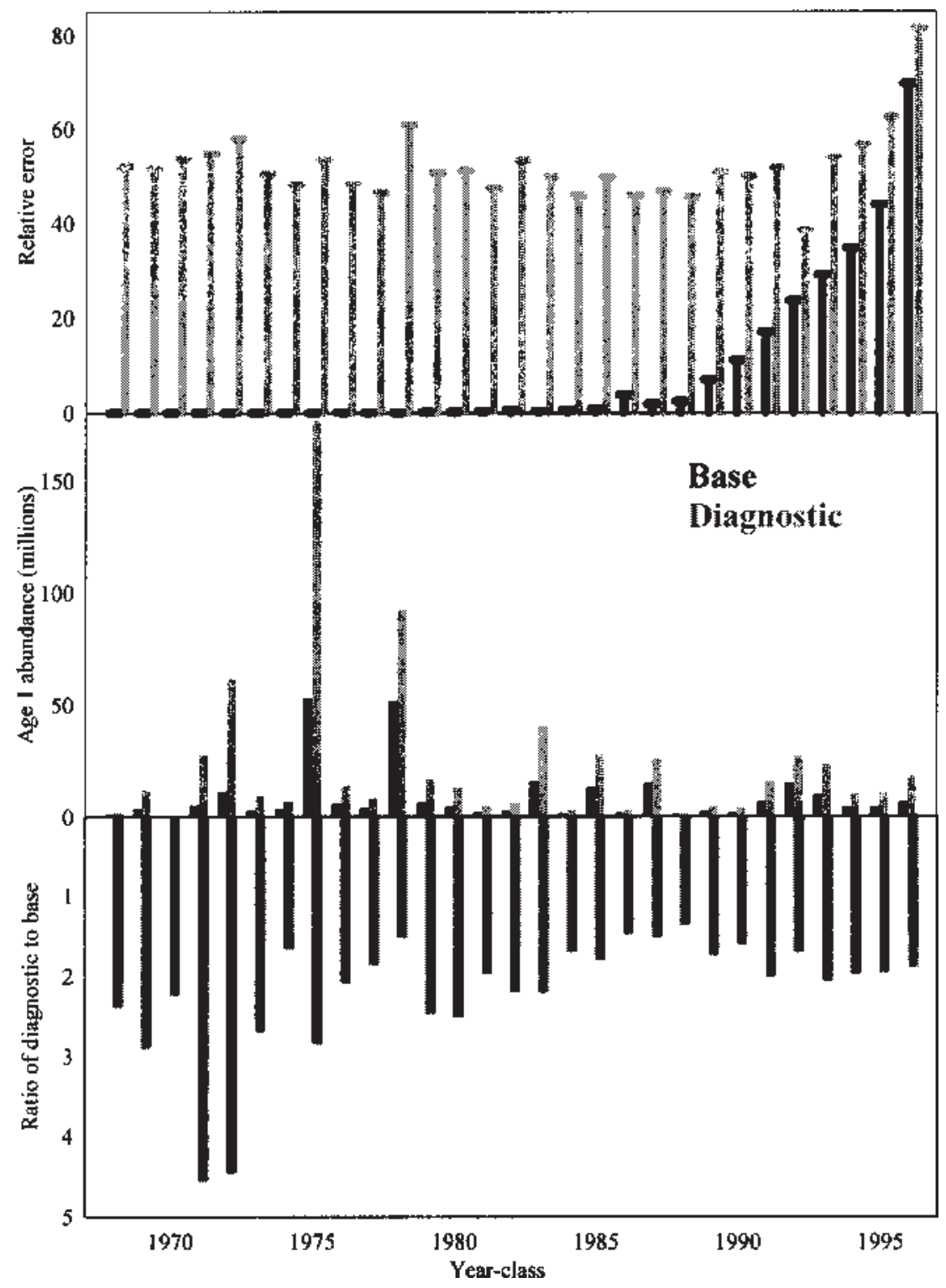

Figure 2. Absolute (middle) and relative (bottom) year-class abundance at age 1 from base and diagnostic assessment models for Georges Bank haddock data, and relative error (standard error/mean) (top) associated with abundance estimates. The 1971 and 1972 relative estimates from the diagnostic model are proportionally much larger than the average ratio. Relative errors for the diagnostic model are about $50 \%$ for all estimates while the error in the base model diminishes rapidly for earlier year classes. 


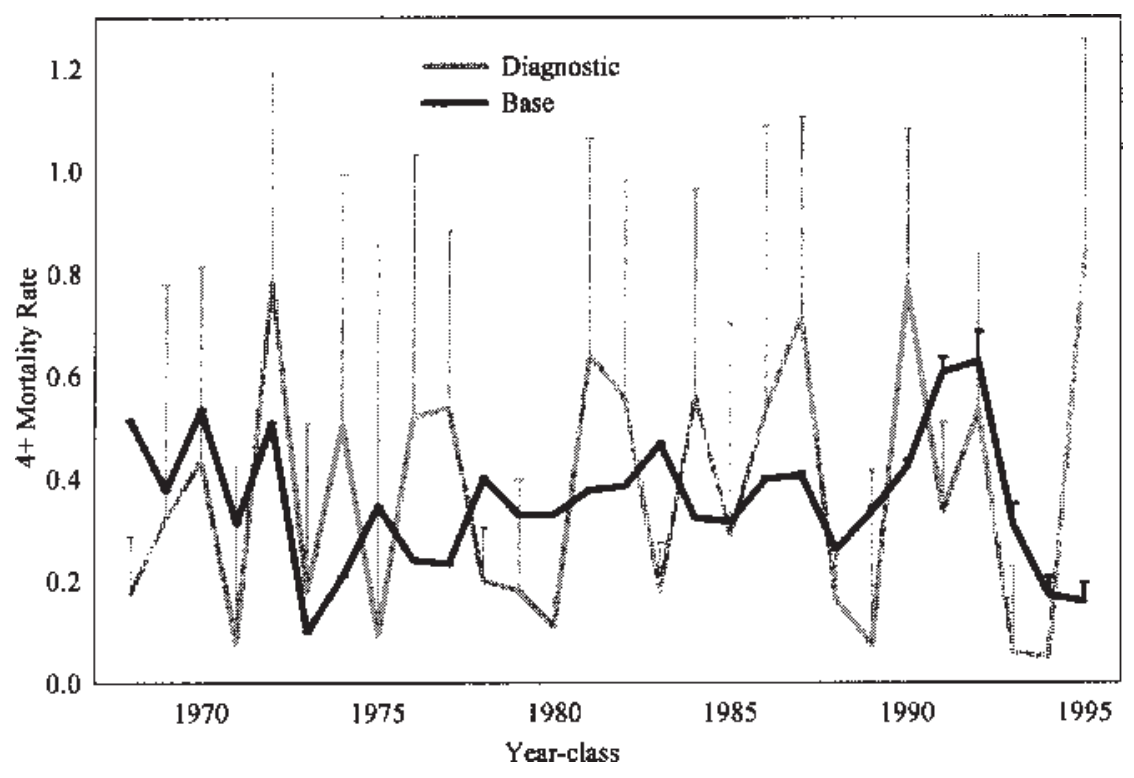

Figure 3. Georges Bank haddock fishing mortality rates for ages which are fully recruited to the fishery, ages 4 to 8 , are much more variable for the diagnostic than for the base model. The relative error (standard error/ mean) on the base model estimates are small and diminish rapidly for earlier year classes while the error on the diagnostic model estimates remain high throughout the time series.

(rank correlation $=0.40$ ), though the general magnitude over the series was comparable for the two. As for the population abundance, the standard errors for the fishing mortality rate from the base model diminish in earlier years but those from the diagnostic model are much larger throughout the time series.

To investigate the sensitivity of the results to the assumed values for the number caught in the seven years where the parameters were assigned fixed values, we conducted two sets of trials. In one set of trials, we halved all the assumed values simultaneously and then we doubled all the assumed values simultaneously. In the former case, all the year-class abundance estimates decreased proportionately while in the latter, they all increased proportionately. Therefore, the relative year-class strengths did not change. In the second set of trials, we selected random multipliers between one half and two and applied them to the fixed values for number caught. The results from 10 such replicates indicated that the yearclass abundance patterns were similar (Fig. 4). Examining the ratio of these 10 trials to the original estimates from the diagnostic model revealed bet- 


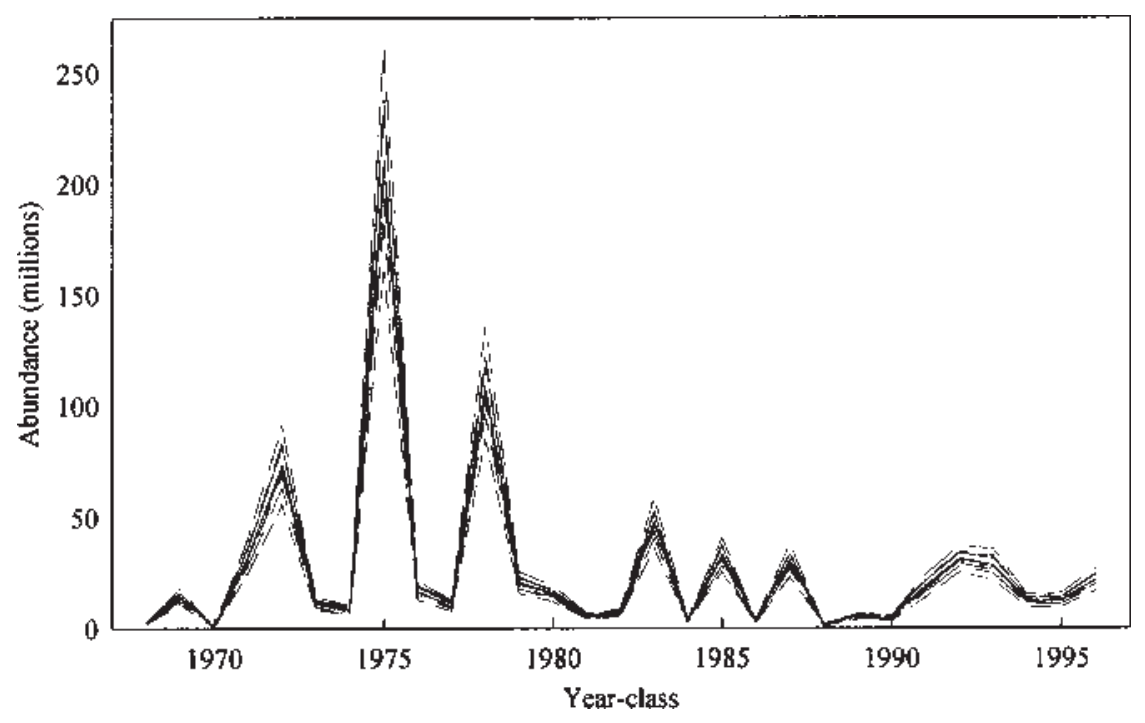

Figure 4. To test the sensitivity of the diagnostic model to fixing the values for numbers caught for several years, the values were varied randomly over a range of half to double the original values. Georges Bank haddock year-class abundance patterns were similar for the 10 trials shown.

ter resolution of the deviations. The ratio lines are essentially horizontal with no persistent slopes (Fig. 5) suggesting that the relative year-class strengths were practically unchanged. Recall that the abundance of the 1992 year class was arbitrarily set to a constant at the beginning of 1997. Therefore, there is limited scope for the abundance of this year class to adjust, and this is evident in the comparison. We concluded from these results that the relative year-class pattern was fairly robust to the assumed fixed values for number caught in those seven years, rendering the comparisons valid.

\section{Discussion}

Statistical models that accommodate random variation do not adequately handle systematic errors in data. Consequently, model results may be inaccurate and unreliable. For fishery stock assessment models, mis-reporting of catches is generally considered to be the most serious source of systematic errors. However, fishery catches play a very important role in the estimation of stock status. In the absence of catch information, absolute estimates of abundance generally cannot be obtained and the relative 


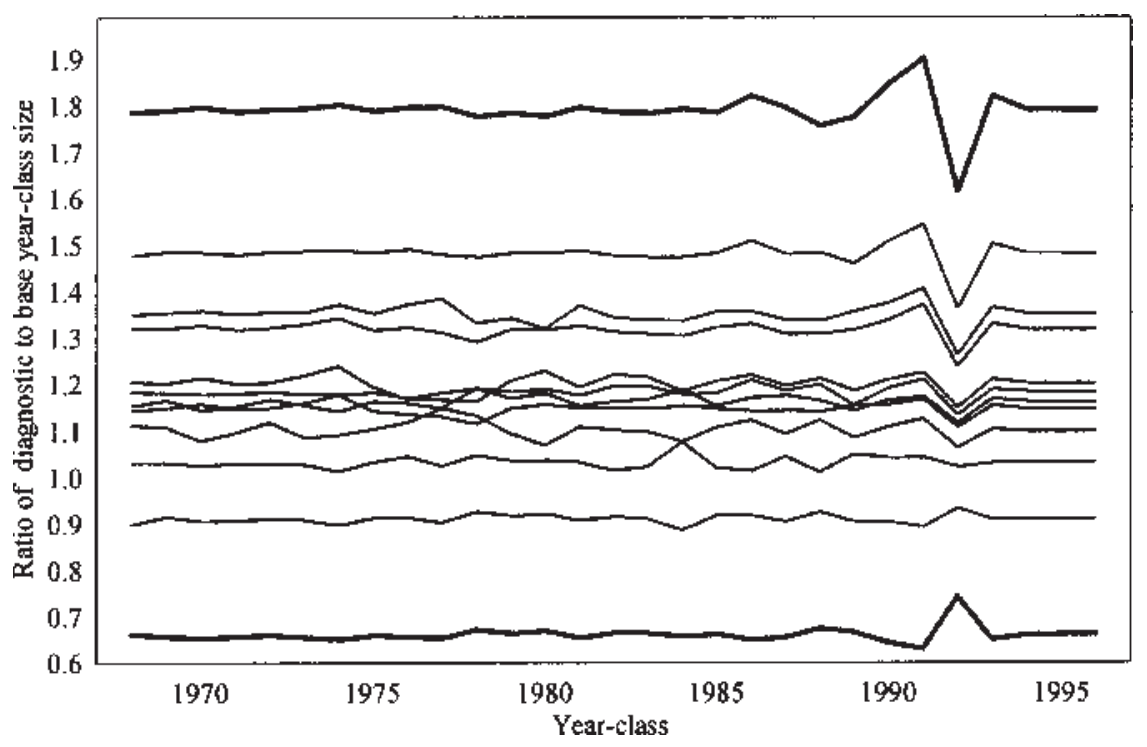

Figure 5. Relative size of Georges Bank haddock year-class abundance estimates from the diagnostic model when the fixed catch values were varied randomly through one half to double the values used in the base model. The ratio of year-class size, diagnostic to base, show that the relative yearclass pattern was fairly robust to the fixed number caught in those select years. The thicker top and bottom lines are the results from doubling (top) and halving (bottom) the base values.

estimates may display large estimation error unless the abundance indices are very precise.

We employed a diagnostic model to identify inconsistencies between results when fishery catches were accepted as accurate and reliable compared to results when those data were not used. We do not advocate use of the diagnostic model for estimation of the status of resources. As anticipated, application of this model to the Georges Bank data demonstrated that the solution might be indeterminate or unstable unless additional assumptions are made. However, the comparison of results suggested that the base model had smaller estimates of the year-class abundance for earlier years. It also indicated that the mid-1980s year classes might have been overestimated by the base model. These observations are useful for focusing attention on specific periods where further investigation of data reliability or robustness to violation of assumptions may be pursued.

For the Georges Bank haddock fishery, 1977 was the last year this resource was fished under an international quota management system. 
Subsequently, the fishery was restricted to coastal states. Differences in pre-1977 and post-1977 monitoring and reporting practices by fleets or possibly intentional mis-reporting by fleets to circumvent restrictive quotas are likely possibilities. The strong 1975 year class would have been susceptible to heavy exploitation at age 2 in 1977 under the international regime. Though estimates of discards for the U.S. fishery have been included, no such adjustments have been attempted for any of the other fleets. The reported catch for this year class at younger ages is probably deficient. Similarly, discard estimates for the U.S. fishery only are included for the 1972 year class, suggesting that the discrepancy for the 1971 and 1972 year classes may have been due to unreported culling and discarding of small fish. Immediately following the establishment of the Canada-U.S. boundary on Georges Bank in 1985, the Canadian fishery in that area was relatively unrestricted. Quotas in the adjacent management unit however, were often restrictive at that time. Fish caught in the adjacent management unit may have been reported as originating on Georges Bank. To prevent this from occurring, fishing vessels now have to choose which grounds they will fish during a trip and they may not fish in both management units on the same trip.

A further avenue that may be pursued is to apply the base and diagnostic models to data for older fish only. For example, in the haddock case, we could apply the models and compare results for ages 3 and older. This may allow us to distinguish between overall misreporting of catches and adequate reporting of landed catch but non-reporting of culled and discarded fish catches.

When the accumulated evidence indicates that fishery catch data may include systematic errors and the assessment results are significantly affected by inclusion of those data, corrective measures should be pursued. In extreme cases where persistent trends are apparent over the time series, it may be prudent to truncate the range of years considered in the analysis to avoid possible non-stationary processes, e.g., decreasing discard rates. For the haddock example, we might investigate the results from an analysis using only the data subsequent to the late 1970s. Another option would be to exclude suspect data points from the objective function. For example, with haddock, we might exclude all data for the 1971, 1972, and 1975 year classes in the minimization. A less severe variant of this would be to include the data but arbitrarily assign a lower weight to those observations. A further option might be to use a hybrid model where the number caught are estimated in only a few years which were suspect while the reported catches were used in all other years. We might use such a model for haddock and estimate the number caught for the years 19851988 only.

We have focused here on biases in the fishery catch data because we have assumed that appropriate measures were taken to avoid systematic deviations in the abundance indices. Differences between the diagnostic 
model and the base model could be caused by systematic patterns in the indices, such as time trends in catchability. Such possibilities should be considered and investigated before the results can be interpreted.

The inability of models, which do not use fishery catch data, to estimate absolute abundance and the high imprecision for estimates obtained from these models limits their use for practical fisheries management advice. It is good practice however, to subject data to models with differing assumptions to evaluate the robustness of results. In this regard, we recommend the diagnostic model as a useful investigative tool, which can uncover important inconsistencies.

\section{Acknowledgments}

We are grateful to the anonymous reviewers for many useful comments and suggestions.

\section{References}

Beverton, R.J.H., and S.J. Holt. 1957. On the dynamics of exploited fish populations. Fish. Invest. Ser. II, 19. 533 pp.

Box, M.J. 1971. Bias in nonlinear estimation. J. R. Statist. Soc., Ser. B 33:171-201.

Deriso, R.B., T.J. Quinn II, and P.R. Neal. 1985. Catch at age analysis with auxiliary information. Can. J. Fish. Aquat. Sci. 42:815-824.

Fournier, D., and C.P. Archibald. 1982. A general theory for analyzing catch at age data. Can. J. Fish. Aquat. Sci. 39:1195-1207.

Gavaris, S. 1988. An adaptive framework for the estimation of population size. Can. Atl. Fish. Sci. Adv. Comm. (CAFSAC) Res. Doc. 88/29. 12 pp.

Gavaris, S. 1993. Analytical estimates of reliability for the projected yield from commercial fisheries. In: S.J. Smith, J.J. Hunt, and D. Rivard (eds.), Risk evaluation and biological reference points for fisheries management. Can. Spec. Publ. Fish. Aquat. Sci. 120:185-191.

Gavaris, S., and C.A. Gavaris. 1983. Estimation of catch at age and its variance for groundfish stocks in the Newfoundland region. In: W.G. Doubleday and D. Rivard (eds.), Sampling commercial catches of marine fish and invertebrates. Can. Spec. Publ. Fish. Aquat. Sci. 66:178-182.

Gavaris, S., and L. Van Eeckhaute. 1997. Assessment of haddock on eastern Georges Bank. DFO Canadian Stock Assessment Secretariat Res. Doc. 97/54. 72 pp.

ICES. 1997. Report of the comprehensive fishery evaluation working group. ICES C.M. 1997/Assess:15. 140 pp.

Kennedy Jr., W.J., and J.E. Gentle. 1980. Statistical computing. Marcel Dekker, New York. 591 pp.

Pope, J.G. 1972. An investigation of the accuracy of virtual population analysis using cohort analysis. Int. Comm. Northwest Atl. Fish. Res. Bull. 9:65-74. 
Ratkowsky, D.A. 1983. Nonlinear regression modeling. Marcel Dekker, New York. $276 \mathrm{pp}$.

Sampson, D.B. 1988. The stability of virtual population analysis cohort size estimates. J. Cons. Cons. Int. Explor. Mer. 44:135-142.

Sinclair, A., and G. Chouinard 1992. Application of a multiplicative model to research survey data for two cod stocks. Can. Atl. Fish. Sci. Adv. Comm. (CAFSAC) Res. Doc. 92/66. 25 pp.

Sinclair, A., D. Gascon, R. O’Boyle, D. Rivard, and S. Gavaris. 1991. Consistency of some northwest Atlantic groundfish stock assessments. Northwest Atl. Fish. Organ. Sci. Counc. Stud. 16:59-77. 


\title{
Model Misspecification or Data Bias: The Assessment of Prince Rupert Herring
}

\author{
Jacob F. Schweigert
}

Fisheries and Oceans Canada, Pacific Biological Station, Nanaimo, B.C., Canada

\begin{abstract}
Pacific herring stock assessments have been conducted with two analytical models since the early 1980s. Correspondence between the estimates of abundance provided by these independent assessments of stock size has been good for four of the five major stock groups. In the remaining area, the Prince Rupert assessment region, estimates of abundance from these models have diverged widely, particularly in recent years. An investigation of the biological sampling data for this area revealed limited information on age structure from the fall food and bait fisheries of the late 1970 s and early 1980s. In addition, there were shifts in the pattern of sample collection throughout the region with time which could be responsible for a bias in the estimates from the age-structured or catch-age model. Deletion of the fall samples from the input data resulted in an improvement in model fit. An investigation of possible bias in the spawn index, used to tune the age-structured model, due to changes in survey methodology yielded minor improvements in model fit and may be artifactual rather than real. Analyses based on the reduced data series described here appear to provide a more realistic reconstruction of stock size and trajectory than in the past and should be used in future assessments of the herring population. This paper provides an example of the benefits of exploring the value and consistency of data inputs or sources.
\end{abstract}

\section{Introduction}

The assessment of Pacific herring stocks in British Columbia has relied on two models to estimate and forecast stock size since the early 1980s. The first model is a modification of the escapement model described by 
Schweigert and Stocker (1988) and reconstructs abundance from an assessment of egg deposition, age composition, and weight-at-age data. The second is a catch-at-age or age-structured model (Fournier and Archibald 1982). These two models have provided similar estimates of absolute abundance and stock trajectories for four of the five major herring assessment regions in British Columbia. In the remaining assessment region, Prince Rupert, the age-structured model estimate of abundance has deviated substantially from the escapement model estimate of stock size in recent years. The age-structured model estimate greatly exceeds the stock size determined from egg deposition data, as well as from the qualitative inseason sonar and sounder estimates of abundance, to the extent that this estimate is not considered in the decision making process for determining annual harvestable surplus. The objective of this study is to revisit the biological data available for this assessment region to determine whether the observed discrepancy in stock trends is a function of model misspecification or bias in the underlying catch-at-age data, with the aim of providing a more realistic assessment of abundance for this area.

\section{Methods}

\section{Stock Considerations}

The geographic scope of the Prince Rupert herring assessment region is shown in Fig. 1 detailing the herring sections which are used to summarize biological characteristics of the stock at finer spatial scales within the region (Haist and Rosenfeld 1988). The major spawning sites for herring in this region occur near Port Simpson in section 42 and in Kitkatla in section 52. In recent years there has been an increase in spawning at Port Simpson and a decrease at Kitkatla. This has led to speculation that the two sites represent distinct herring stocks. However, two separate tagging studies have demonstrated considerable movement of fish between these and other areas (Stevenson 1954, Haegele 1991). Also, suggestions have been made that some of this stock may move north across the British Columbia-Alaska border to mix with the Kah Shakes herring stock. The available biological data do not indicate any large scale unidirectional movement of herring north or south, although tagging has indicated some small scale movement between these areas. For the purposes of this study, the Prince Rupert assessment region is assumed to represent a distinct biological entity.

\section{Assessment Models}

The two assessment models, an "escapement" model and an "age-structured" model, used to assess British Columbia herring stocks, have been described in detail by Schweigert et al. (1997). The escapement model, used since 1984, relies on an assessment of the escapement from the fishery plus total catch to estimate the pre-fishery spawning stock biomass 


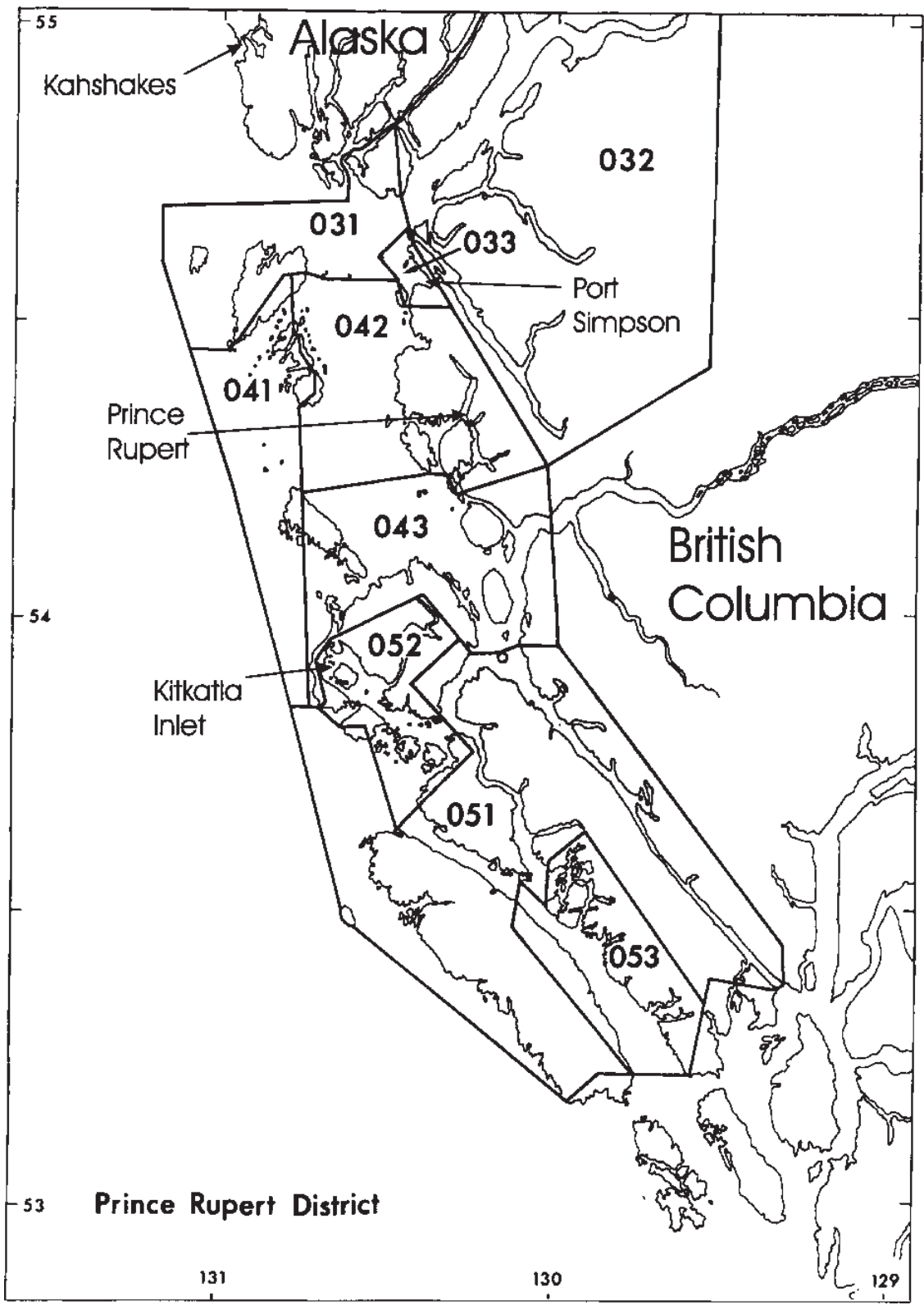

Figure 1. Herring sections in the Prince Rupert District which define the assessment region and delineate the geographic subunits for data aggregation. 
for each assessment region. The estimated escapement to each region is derived from information on spawn deposition. Total egg deposition for each spawning bed or location is calculated as the product of: total length of the bed, an estimate of the observed or adjusted width of the bed, and an estimate of the egg density determined from a visual assessment of the number of layers of eggs on the available vegetation substrates (Schweigert and Stocker 1988, Schweigert 1993). Prior to 1988 all estimates of these parameters of the egg beds were determined from the surface of the water either from a skiff using a grapple or on foot at low tide. Subsequently, scuba surveys of spawning locations have been used to quantify the egg deposition and historical surface observations have been adjusted to emulate more recent diving data. Egg deposition estimates for all spawn beds are summed within each assessment region and the total egg deposition is converted to tons of spawning fish based on an estimate of 100 eggs per gram of herring (Hay 1985).

The age-structured model has been used to assess B.C. herring stocks since 1982. Ongoing revisions to the model have made it more consistent with the life history of herring and the fisheries. The current version uses auxiliary information on spawning escapement, separates catch and age composition data by gear type, and includes availability parameters to estimate partial recruitments to the spawning stock. Model parameters are estimated simultaneously using a maximum likelihood method. The model is implemented in the $\mathrm{C}++$ programming language using AUTODIF for derivative calculations (Otter Research Ltd. 1992).

Two types of fishing gear are used commonly in B.C. herring fisheries. Seine nets are assumed to be nonselective while gillnets are selective for larger, older fish. Herring fisheries have concentrated primarily on fish which are on, or migrating to, the spawning grounds. Therefore, the relative availability of age classes to nonselective gear should be equivalent to the partial recruitment of age classes to the spawning stock. The agestructured model explicitly separates availability (partial recruitment) and gear selectivity. Seine and gillnet fisheries are temporally separate so catch and age composition are partitioned into fishing periods, separating data for the different gears. Three fishing periods are modeled. The first period encompasses all catch prior to the spring roe herring fisheries. This includes reduction fishery catches prior to 1968 and the winter food and bait fisheries since 1970. Most of this catch was taken by seine gear although small amounts were caught with trawl nets (which are also assumed to be non-size selective). The second fishing period includes all seine roe herring catch and the third period includes all gillnet roe herring catch.

Briefly, assume $T_{i j}$ is the total number of fish in age class $j$ at the beginning of season $i$, where season is equivalent to year, and $\lambda_{i j}$ is the proportion of age $j$ fish which are available to the fishery. Then, $N_{i j 1}$ the total number of age class $j$ fish which are available at the start of period 1 in season $i$ is given by 


$$
N_{i j 1}=\lambda_{i j} T_{i j}
$$

To model the fishing process a form of the catch equations, which models fishing and natural mortality as continuous processes over time period $r$, is used:

$$
C_{i j r}=\frac{F_{i j r}}{F_{i j r}+M_{r}}\left[1-\exp \left(-F_{i j r}-M_{r}\right)\right] N_{i j r}
$$

and, for $r<p$

$$
N_{i j r+1}=N_{i j r} \exp \left(-F_{i j r}-M_{r}\right)
$$

where

$C_{i j r}$ is the catch of age class $j$ in season $i$ for period $r$,

$F_{i j r}$ is the fishing mortality of age class $j$ in season $i$ for period $r$,

$M_{r}$ is the natural mortality for period $r$,

$N_{i j r}$ is the number of fish in age class $j$ in season $i$ for period $r$,

$p$ is the number of fishing periods $(p=3)$,

$n \quad$ is the number of seasons $(n=47)$,

$k \quad$ is the number of age classes $(k=9)$.

$N_{i+1, j+1,1}$ is defined by equation (4) where for $j+1<k$

$$
T_{i+1, j+1}=N_{i j p} \exp \left(-F_{i j p}-M_{p}\right)+T_{i j}\left(1-\lambda_{i j}\right) \exp \sum_{r}-M_{r},
$$

In the model the last age class, $k$, accumulates all fish aged $k$ and older. To reduce the number of parameters to be estimated assumptions are made about the form of the availabilities and mortalities. The availabilities are formulated to increase with age and are set to 1 for age $6+$ and older. For age $3+$ to $5+$ the availabilities are assumed to be constant between years. Because the proportion of age 2+ fish that are mature appears to vary among years (Haist and Stocker 1985) and some reduction fisheries targeted on immature 1+ fish, the availabilities for these two age classes are estimated for each year for which there is age-composition data with the exception of the final year.

For the selective gillnet fishery (i.e., fishing period 3), fishing mortality is separated into age selectivity and fishing intensity components. For 
nonselective fisheries (i.e., fishing periods 1 and 2) only fishing intensity parameters are estimated. A natural mortality parameter, $M_{\text {., }}$ is also estimated. Natural mortality for the three fishing periods is modeled as,

$$
\begin{aligned}
& M_{1}=0.95 M . \\
& M_{2}=M_{3}=0.025 M .
\end{aligned}
$$

Additional structure is built into the model through the inclusion of annual spawn data (spawn index, $I_{i}$ ). Spawning occurs at the end of the season so the number of spawners at age $j$ in season $i\left(G_{i j}\right)$ is estimated by

$$
G_{i j}=N_{i j p} \exp \left(-F_{i j p}-M_{r}\right)
$$

and the spawning stock biomass, which is assumed to be equivalent to egg production, in season $i\left(R_{i}\right)$ is

$$
R_{i}=\sum_{j} w_{i j} G_{i j}
$$

where $w_{i j}$ is the average weight-at-age $j$ in season $i$. The errors in the spawn index observations $\left(I_{i}\right)$ are assumed to be multiplicative so that

$$
I_{i}=q R_{i} \exp \left(\xi_{i}\right)
$$

where $q$ is a spawn conversion factor and $\xi_{i}$ is a normally distributed random variable with mean 0 and variance $\sigma_{1}^{2}$.

\section{Data Considerations}

Biological data on total catches, weight at age, age structure, and egg deposition are available from 1951 to present. From these data, catch at age and total egg deposition data were determined and total stock abundance calculated from age-structured and escapement model analyses as described above. The results of applying the age-structured model to three alternative sets of input data are presented below. First, consider all the available data assuming no data biases which is the current model. Second, accept that the catches and associated biological samples collected from fall fisheries since the beginning of the roe fishery in 1972 are limited and may not be representative of the selectivity pattern that occurred during the earlier reduction fishery or of the current roe fishery, and therefore remove these data from the analysis. Third, delete potentially biased samples as above but also adjust the spawn index or egg deposition data since 1988 for a possible 25\% overestimate of spawn deposition from diving surveys relative to previous surface based spawn assessments due to increased survey effort and efficiency. 


\section{Results}

Trends in abundance for the age-structured model estimate of stock size and for the escapement model or the egg deposition index are presented in Fig. 2. The results indicate good agreement between the escapement and the age-structured estimates for all areas except the Queen Charlotte Islands and Prince Rupert. It is generally agreed that because of its remoteness, some spawnings in the Queen Charlotte Islands were not completely surveyed in the 1970s and early 1980s. However, with the beginning of diving surveys in 1988, spawn surveys are felt to be representative of the total spawn deposition in the area and these results agree with the age-structured assessment since that time. The same is not true of the Prince Rupert assessment where there is poor agreement with the escapement estimate from the early 1970s through the mid-1980s and again in recent years. Because of its proximity to the city of Prince Rupert it is assured that no significant spawning events would have been missed during the annual spawn assessments throughout this period (Pers. comm., L. Gordon, Dept. Fisheries and Oceans, Pt. Alberni, B.C.). Hence, the discrepancy between the two abundance indicators has been difficult to reconcile.

The results of conducting a retrospective analysis for these data from the age-structured model further supports these findings (Fig. 3). The assessment of stock size for all areas except Prince Rupert has been consistent over time in a retrospective sense although there has been a tendency to underestimate abundance in the two southern assessments. For Prince Rupert the agreement between the models has become worse for the period from 1970 to 1985 as new data have been added to the analysis, and somewhat better thereafter as the estimate of stock size by the age-structured model has declined.

In an effort to reconcile the two estimates of abundance the biological sampling data were reviewed. It was noted that the spatial and temporal distribution of the sampling effort had changed substantially over time. During the reduction fishery prior to 1972, sampling effort was fairly evenly distributed throughout the assessment region with most samples coming from sections 41 or 42 and 51 (Fig. 1). With the advent of the roe fishery in the 1970s, which focused on the spawning grounds, sampling effort became more concentrated spatially in sections 33 or 42 and 52 with the majority coming from the latter area. In the current analysis it is assumed that all available biological samples are representative of the mature population and so contribute equally to the estimate of catch at age. Therefore, to identify possibly unrepresentative or biased samples, a variety of ordination methods were investigated as a means to detect outliers in the data series for each year that might be contributing to the poor fit of the age-structured model to the catch-at-age data. However, because there was no objective basis for rejecting any particular sample in the data series this approach was not very useful for identifying biased biological 


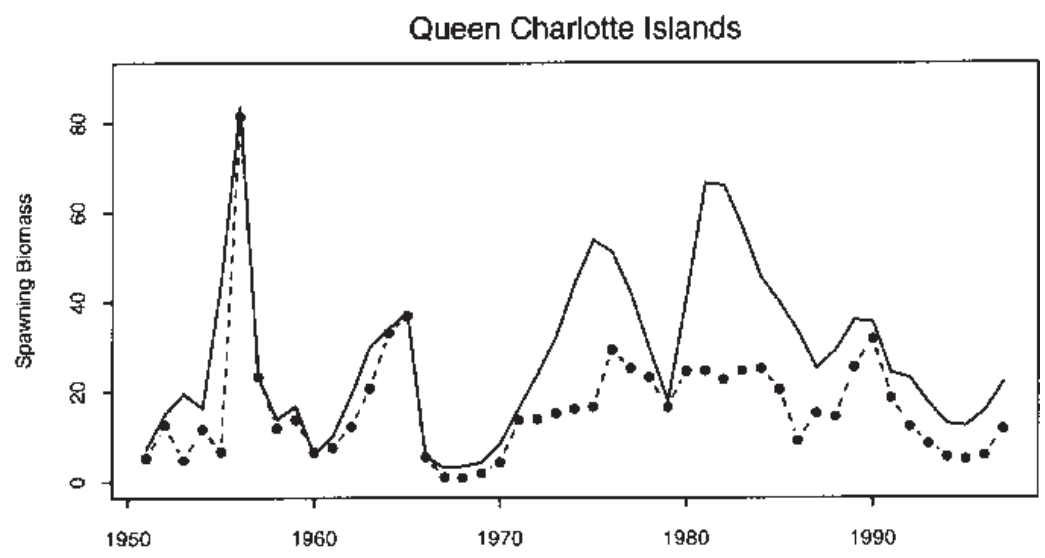

Prince Rupert District

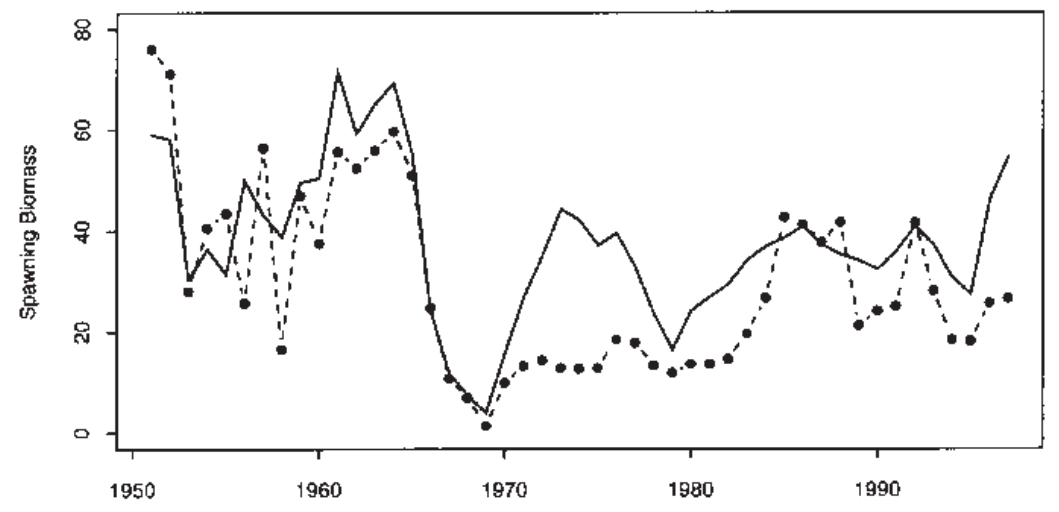

Central Coast

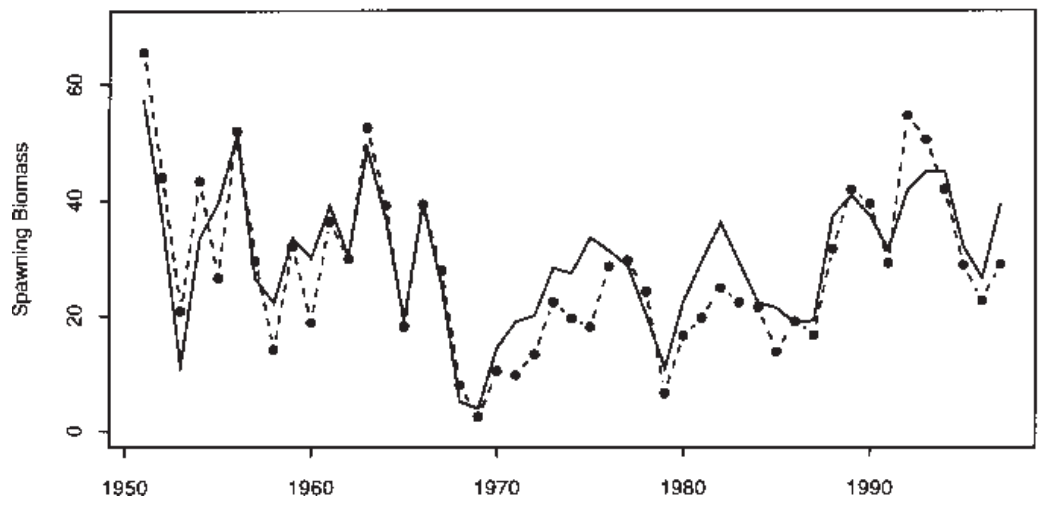

Figure 2a. Estimates of pre-fishery spawning stock biomass (1,000 t) from agestructured (solid line) and escapement model (dashed dotted line) analyses for northern B.C. herring stock assessment regions, 1951-1997. 

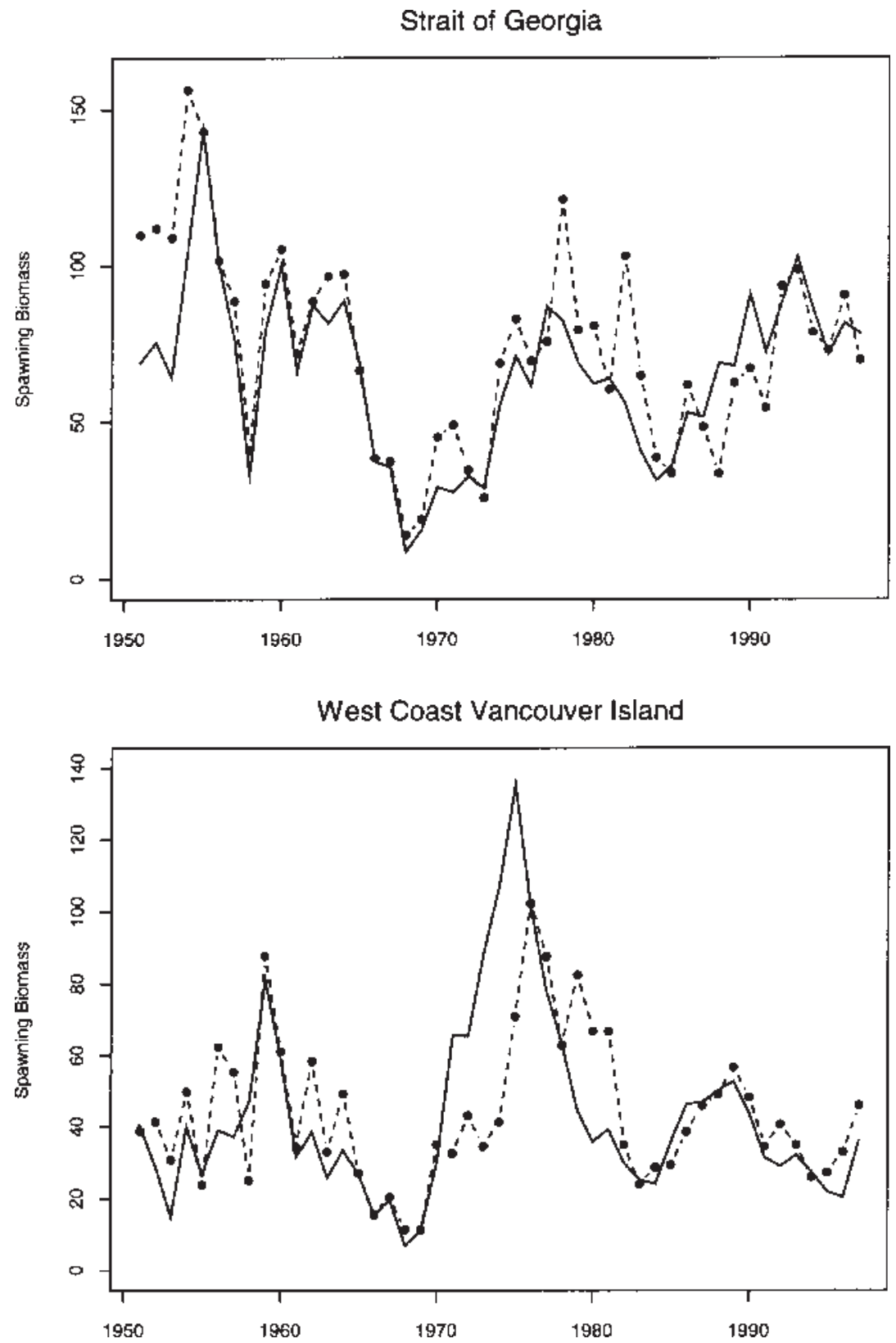

Figure $2 b$. Estimates of pre-fishery spawning stock biomass (1,000 t) from agestructured (solid line) and escapement (dashed dotted line) model analyses for southern B.C. herring stock assessment regions, 1951-1997. 


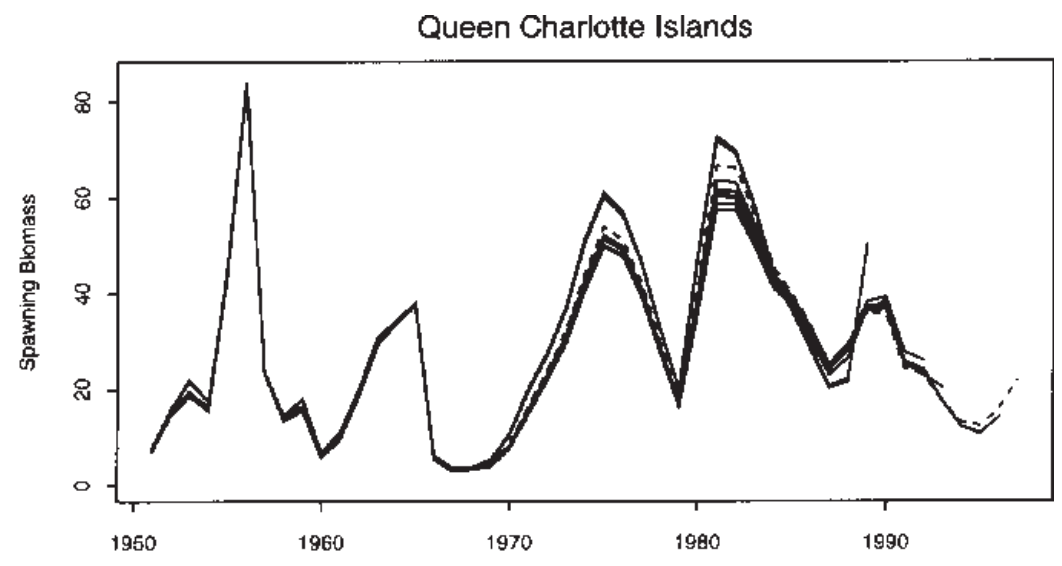

Prince Rupert District

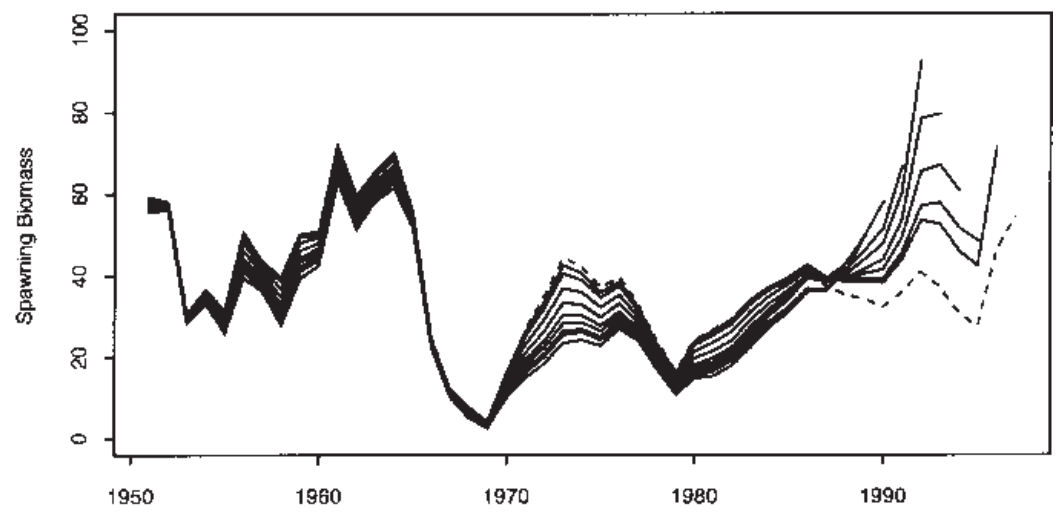

Central Coast

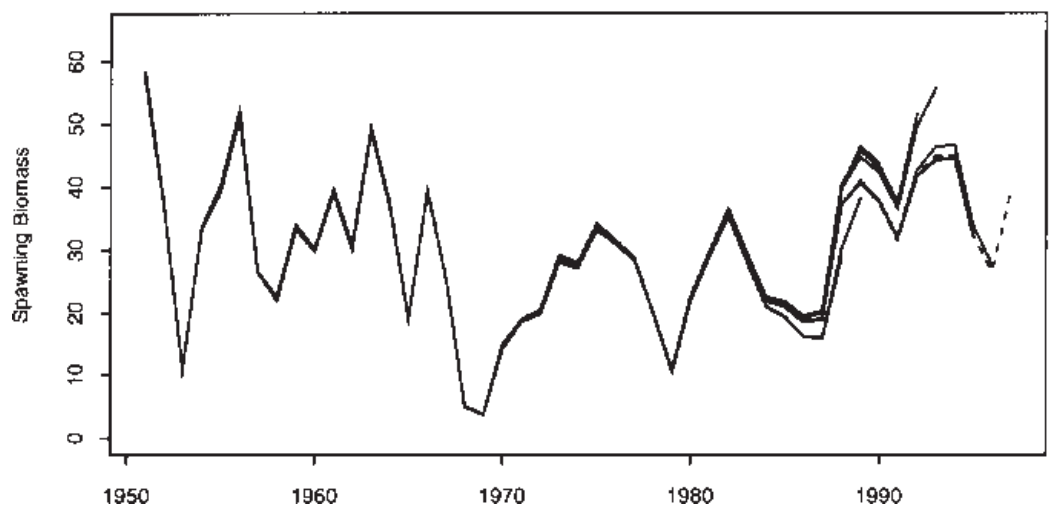

Figure 3a. Retrospective analysis of estimated spawning biomass $(1,000 \mathrm{t})$ from age-structured analysis for northern B.C. herring stocks, 1951-1997. 

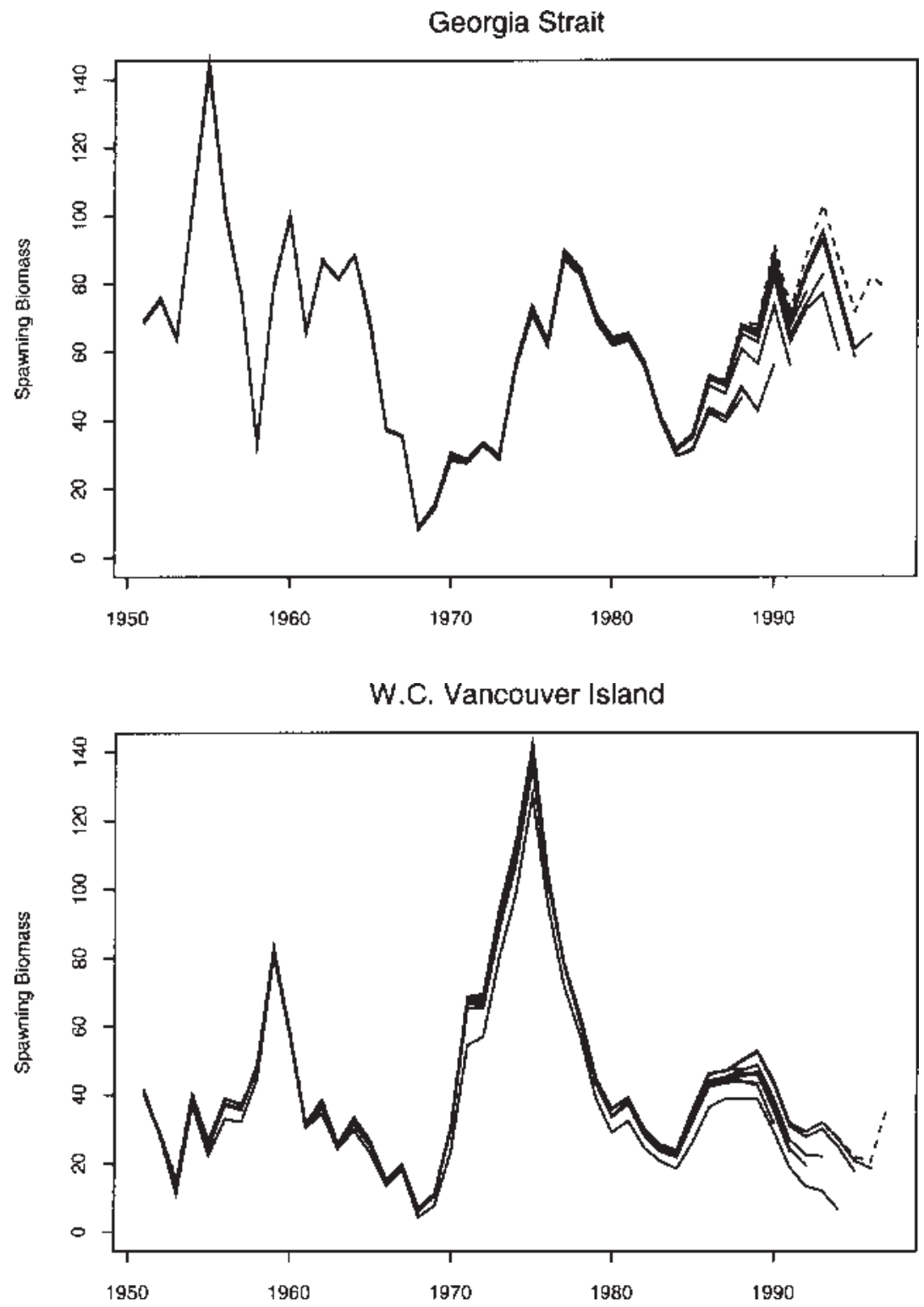

Figure 3b. Retrospective analysis of estimated spawning biomass (1,000 t) from age-structured analysis for southern B.C. herring stocks, 1951-1997. 
samples. Instead, the estimated age compositions from all the available samples for each of the three fishing periods (reduction seine, roe seine, and roe gillnet) were examined. These data highlighted significant differences between the estimates of age structure of the catch depending on when and where the samples were collected (Fig. 4). It is evident that, for a number of the years for which samples are available from both a fall food or bait fishery and a subsequent spring seine roe fishery, the same population is not being sampled (1973, 1976-1979, 1982, 1985, 1986). In fact, the graphs indicate that the fall food and bait samples do not reveal the dominant cohorts of fish that are found in the spawning run the following spring and as a result are apparently biasing the age-structured assessment of stock abundance and trend.

To examine the impact of the fall samples, these data were dropped from the data set and the catches taken in these fisheries were combined with the roe catches the following spring. The roe fishery sampling data then became the only source of catch-at-age information for the age-structured analysis. The result of this analysis is shown in Fig. 5 where the abundance time series is compared to the current analysis and the escapement model estimate. The resulting fit to the catch-age data from 1970 to 1980 remains similar although abundance is markedly lower from 1972 to 1974 . However, from 1981 to 1990 the estimate of abundance increases significantly and subsequently more closely approximates the magnitude and trend in the escapement model estimate of stock size.

In addition to this analysis, the impact of a possible systematic bias in the spawn index due to a change in data collection techniques was investigated. Beginning in 1988, the assessment of egg deposition has increasingly relied on diving surveys since much of the spawn occurs subtidally and was likely underestimated by earlier surface based surveys. While historical data have been adjusted to account for this effect (Schweigert et al. 1997), it is conceivable that the use of scuba based surveys has discovered more egg beds than was the case previously, and as a consequence the spawn index is biased upward due to increased survey effort and efficiency. To evaluate this possibility the spawn index data used in the agestructured analysis were arbitrarily deflated by $25 \%$ to render them more similar to what might have been observed using historical survey procedures, and the analysis was repeated on the reduced sampling data set (no food and bait samples after 1972). The biomass trajectory for this analysis is also presented in Fig. 5 and closely follows that for the reduced sampling data set until about 1985 when the fit more closely approximates the escapement model abundance series.

The residuals from the current age-structured analysis for the fit to the age composition data are shown in Fig. 6a. For comparison, the residuals from the fit to the reduced sampling data set are presented in Fig. 6b. A similar plot for the adjusted spawn analysis does not result in any detectable difference from Fig. $6 \mathrm{~b}$ and so is not presented here. It is clear from Fig. $6 \mathrm{~b}$ that removal of the food and bait samples from the analysis 

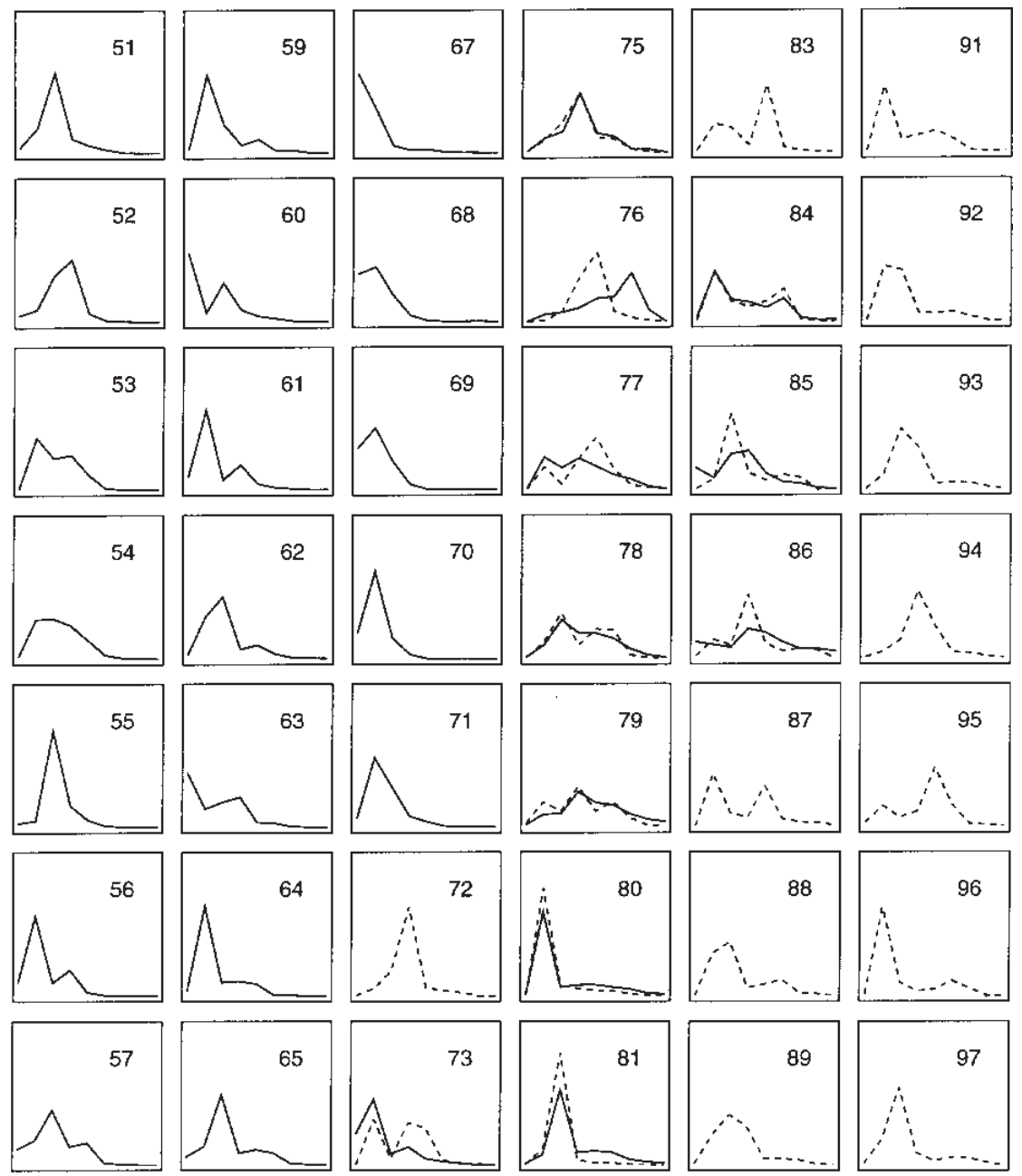

58
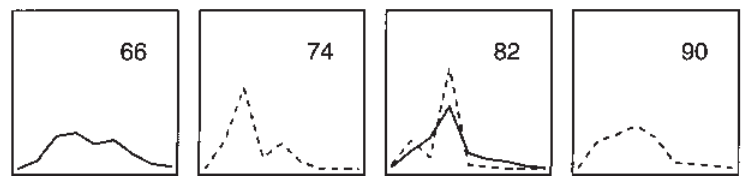

Figure 4. Estimated age composition by year for period 1 (fall fisheries $=$ solid line) and period 2 (spring fisheries = dashed line) in the Prince Rupert region, 1951-1997. Ordinate ranges from zero to one and abscissa from ages 1 through 10. 


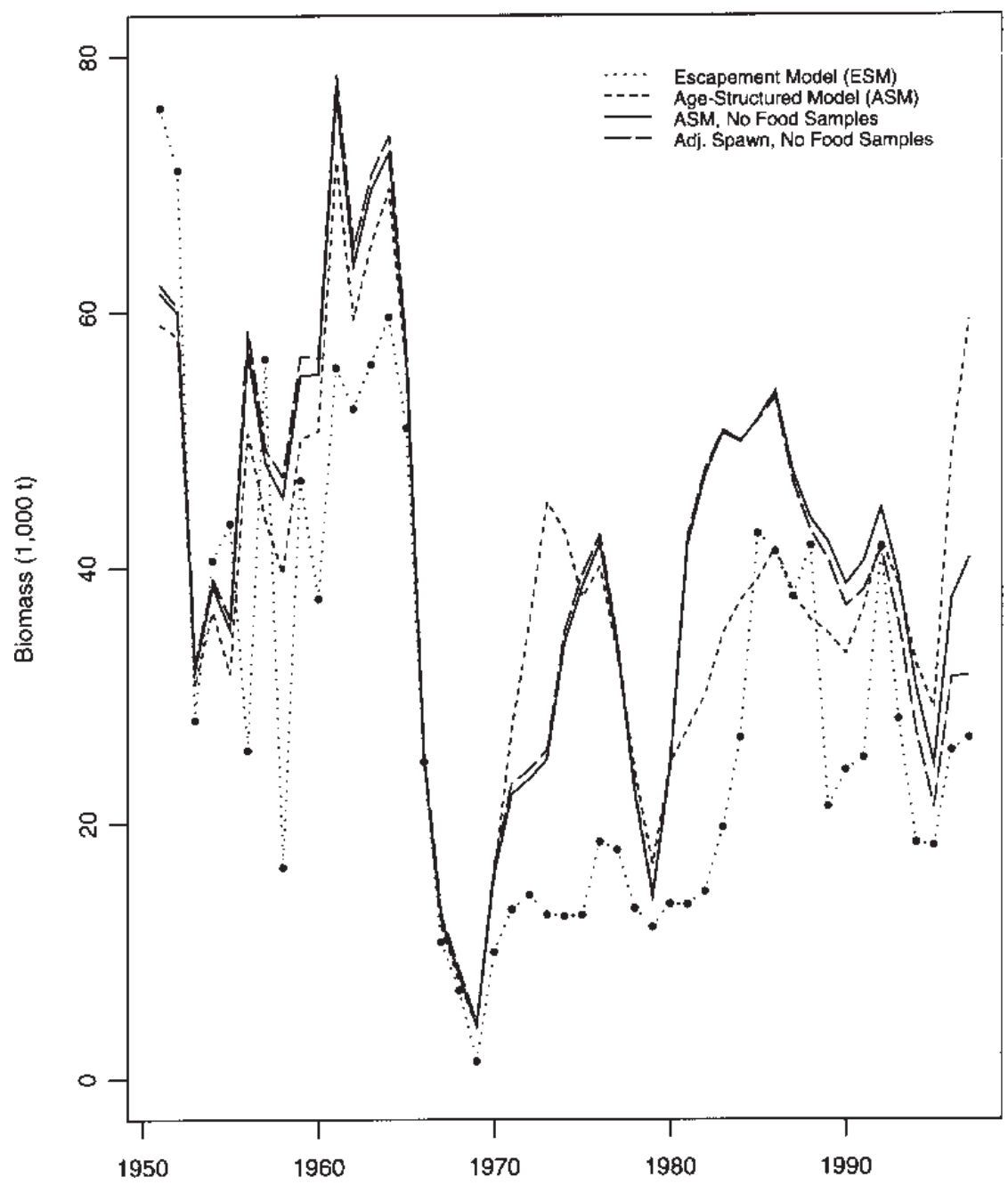

Figure 5. Estimates of mature biomass for age-structured model analysis, for an adjusted data set removing fall food fishery samples since 1972, and for an adjusted spawn index without fall food samples, relative to the escapement model estimate for 1951-1997. 

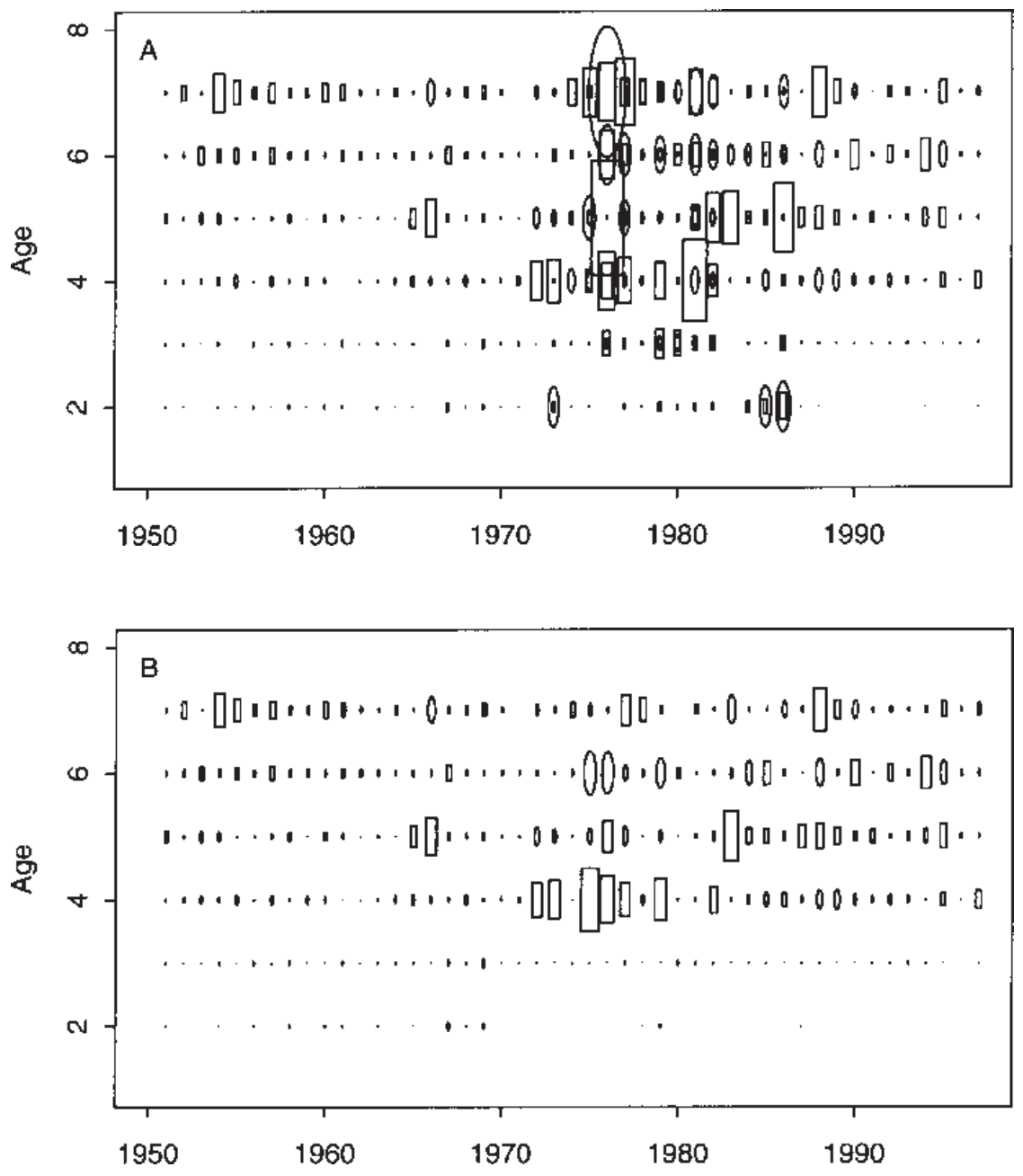

Figure 6. Standardized residuals from the age-structured analysis using (A) the current model and (B) the reduced data set without fall food fishery samples after 1972. Positive residuals are elliptical and negative values appear as rectangles. Multiple residuals for year and age combinations reflect the presence of both fall and spring samples in A, from which fall samples are removed in $B$. 
has markedly improved the fit to the age structure data for this assessment region.

The lognormal residuals from the fit of the age-structured model estimate of egg production (equation 8) and the observed spawn index data are plotted in Fig. 7. Results indicate a generally improved fit to the spawn index over much of the time series, except the period 1981-1984, for the reduced sampling data set relative to the base analysis. There is also a marked deviation of the model estimates from the spawn index for the 1950s which may have other explanations such as incomplete spawn surveys, poor catch reporting, or fisheries targeting on mixtures of fish destined for other areas. The adjustment of the spawn index to assess the impact of changes in survey methodology is equivocal since there is no marked improvement in the fit to the spawn index; in fact, the fit is actually poorer in many of the recent years.

The estimated minimum function value determined for each analysis is presented in Table 1 for all assessment regions and for the two alternate analyses described here. It is apparent that the current assessment for Prince Rupert has provided a significantly poorer fit to the available data than has the same model in any of the other areas. The fit of the Prince Rupert assessment region without the food and bait sampling data is significantly better than the base assessment and in the range of that observed for other stocks. The fit of the model to the reduced data set and the adjusted spawn is marginally better than to the reduced data set alone. For comparison, the fit to the current data series without the roe seine sampling data from section 52 also shows a very marginal improvement in the fit for this stock relative to the base assessment. The modified analysis was presented in a recent assessment to adjust for apparent overweighting of sampling from section 52 relative to other sections in the region in recent years (Schweigert et al., unpubl. manuscript).

\section{Discussion}

An assessment of the status of any resource relies on the development of a quantitative model of the processes that describes changes in the dynamics of the system with time. The model may then be tested against the available data to estimate the parameters of interest. An important question that frequently arises in the evaluation of the adequacy of the model is whether the mathematical structure or relationships hypothesized in constructing the model are supported by the available data or if perhaps there are errors or biases in these data or there are insufficiencies in the model structure. Generally, it is not possible to decide between these alternatives although there are subjective approaches for evaluating alternative competing models, such as comparing the function values of a class of models at their respective maxima or comparing the parameter estimates from competing models against independent information that was not used in constructing the model. Often, however, there are systematic 


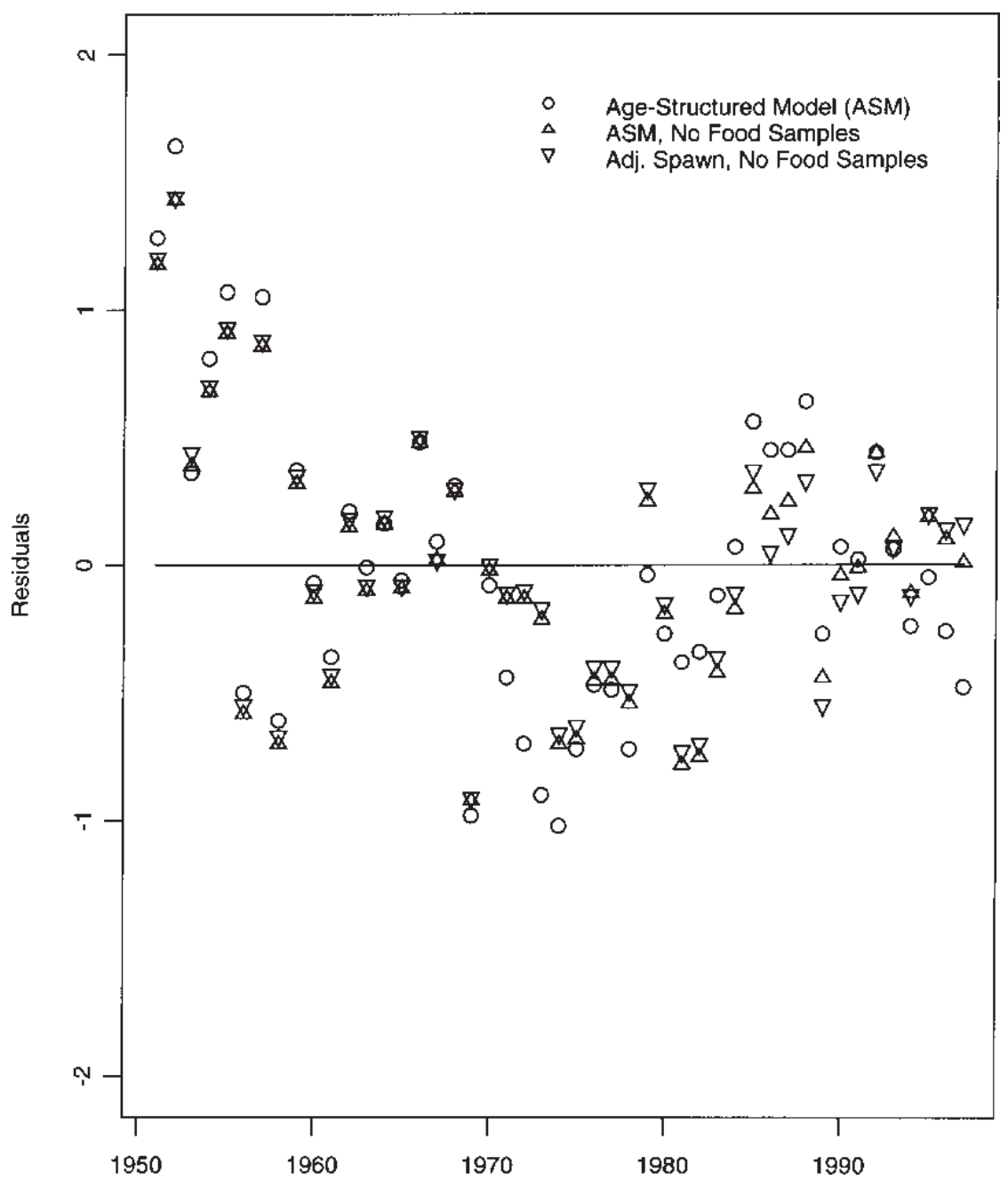

Figure 7. Estimated lognormal residuals from the relationship between the observed spawn index and the total egg production derived by the current agestructured model, for a reduced model without fall food fishery samples since 1972, and for an adjusted spawn index without fall food samples. 
Table 1. Estimated minimum function values from age-structured model analysis for British Columbia herring stocks from 1951 to 1997.

\begin{tabular}{lcc}
\hline & Function value & No. of parameters \\
\hline Queen Charlotte Islands & 582 & 199 \\
Prince Rupert base model & 2,652 & 233 \\
$\quad$ Reweight sect. 52 samples & 2,222 & 233 \\
$\quad$ No fall food samples (NFFS) & 1,152 & 221 \\
$\quad$ Adjusted spawn (NFFS) & 1,148 & 221 \\
Central coast & 520 & 222 \\
Georgia Strait & 1129 & 249 \\
W.C. Vancouver Is. & 935 & 207 \\
\hline
\end{tabular}

biases in the available biological data used to assess the resource that have effects on model performance but are difficult to interpret or to adjust for in model restructuring. The present study attempts to discern some of these effects for one of the British Columbia herring stocks using a combination of methods.

The age-structured model used in the assessment of British Columbia herring stocks evolved over a period of years through a trial and error process which attempted to encapsulate the important population dynamics processes of herring populations and the associated fisheries (Haist et al. 1988). The available data on catch at age and relative stock size in the form of spawn index information are fitted to the model to estimate population parameters of interest and deviations or residuals from the fitted model examined to attempt to understand the process and measurement errors of the model. In the application of this model to the five major herring populations in British Columbia it was evident that an acceptable fit to the spawn index occurred in the three southern populations. A reasonable fit also occurred for the Queen Charlotte Islands for all but the early roe fishery period when there was apparently limited effort devoted to assessing spawn deposition. The fit is improved in recent years where extensive diving surveys have provided good spawn survey coverage. Taken together this result suggests that the current model structure adequately represents the population dynamics of British Columbia Pacific herring and that the lack of fit to the Prince Rupert area data is indicative of data anomalies which are inconsistent with the underlying model assumptions about herring population dynamics.

Richards et al. (1997) recently commented on the applicability of graphical approaches for interpreting catch-at-age analyses and some of these suggestions are adopted here. For example, a simple time series plot of the estimated age structure of the population each year as determined by 
samples from the various fisheries revealed anomalies between the population structure as evidenced in the fall and that observed the following spring (Fig. 4). While it might theoretically be possible to alter the structure of the age-structured model to describe the change in age composition from fall to spring as a function of changes in distribution or migration, the underlying biology is not well understood. It is also possible that the measurement error in the samples which were collected in the fall is so large that these samples do not accurately reflect the true underlying population age structure which is caught in the spring roe fishery, or in fact yields a biased estimate of these parameters. The retrospective analysis conducted for all five herring stocks lends some support to this interpretation because it demonstrates that for all but the Prince Rupert population the stock reconstructions have remained relatively stable, as new data are included in the data set and stock trajectories are re-estimated (Fig. 3). For Prince Rupert the stock reconstruction varies widely suggesting that there are serious inconsistencies in the underlying age composition and consequently catch at age which make it impossible to consistently determine relative cohort strengths over time. As a consequence the stock reconstruction varies widely depending on which new set of data are added to the model and how they alter the estimated fishing intensity parameters. The approach adopted here to deal with this problem was an expedient one since it was determined that the number of samples available for estimating age composition for the years of greatest discrepancy were minimal. Hence, it was simplest to ignore these samples and rely on the estimates of age composition determined from the more extensive sampling program during the spring roe fisheries, to estimate the catch at age for the entire food and roe catch throughout the roe fishery period beginning in 1972 .

It is also recognized that this simple adjustment to the data series, although significantly improving model fit, has not accounted for all of the residual variation in the fit to the data series. For example, it is evident that as the fisheries have focused on roe, sampling has become more concentrated geographically which has had the effect of differentially weighting the age structure and consequently the catch-at-age data to those areas or components of the stock that are most readily available to the sampling gear. Such a practice could also be introducing a bias into the sampling data and the effects of differentially weighting some of the sampling data should be further investigated. It has also been demonstrated that the older fish spawn first so the age structure of the herring run changes during the season (Hay 1985) which may bias the sampling data depending on the synchronicity of the herring spawning times and the sampling program. An attempt to adjust for this effect was presented in a previous assessment for this stock and involved removing all seine fishery samples collected from section 52 since 1970 from the data set (Schweigert et al., unpubl. manuscript). The effect of such a simple reweighting of the catchat-age data was not particularly effective, providing only a marginally better 
fit to the data than the base assessment (Table 1 ). This also raises the question of how important an effect this might be in other assessment regions. The only other area in which a significant fall food and bait fishery occurs is the Strait of Georgia, and it will be necessary to examine these data in a similar manner to determine whether adjustments to the basic data might also improve the model fit for that stock.

An interesting question that remains is why the age-structured and escapement model biomass estimates differ so significantly in the early roe fishery period if survey effort and coverage were thorough and consistent throughout this period. Prior to 1970 harvest rates were generally very large and removed upward of $50 \%$ of the available stocks in most areas of the coast (Hourston 1980). As a result most of the biomass estimated during this period was determined by the catch since there was relatively less spawning escapement, and so the stock abundance estimate should be fairly well determined. Throughout the roe fishery period, harvest rates have been maintained close to the 20\% target (Schweigert et al. 1997) and as a result the biomass estimate is heavily dependant on the estimate of egg deposition. A possible explanation remains that significant herring spawning occurred in unusual areas or at unusual times and as a consequence went unreported and unsurveyed. Another possibility is that the procedures used to convert these data to estimates of spawning biomass are inappropriate for this area, although they seem to work quite well in the other assessment regions. The only other possibility seems to be that there are outstanding biases in the age structure information during the earliest years of the roe fishery that need to be resolved or the model structure altered to account for them if a reliable catch age analysis for this stock is to be obtained.

In summary, based on the compare and contrast strategy adopted here to evaluate model process error, there is no evidence of any serious failure to meet the structural assumptions of the existing catch-age model used in British Columbia herring stock assessments. Removal of a few apparently biased samples of population age structure resulted in a markedly improved fit of the model to catch-age data and spawn index information without the need to alter model formulation for the Prince Rupert assessment region. The base assessment model applied to a reduced data series provides a comparable fit to that for the other B.C. herring stocks and should be used in the future assessment of this resource.

\section{References}

Fournier, D., and C.P. Archibald. 1982. A general theory for analyzing catch at age data. Can. J. Fish. Aquat. Sci. 39:1195-1207.

Haegele, C.W. 1991. Returns from anchor taggings of herring in British Columbia, 1979 to 1985. Can. Data Rep. Fish. Aquat. Sci. 582. 129 pp. 
Haist, V., and L. Rosenfeld. 1988. Definitions and codings of localities, sections, and assessment regions for British Columbia herring data. Can. Manuscr. Rep. Fish. Aquat. Sci. 1994. 123 pp.

Haist, V., and M. Stocker. 1985. Growth and maturation of Pacific herring (Clupea harengus pallasi) in the Strait of Georgia. Can. J. Fish. Aquat. Sci. 42(Suppl. 1):138-146.

Haist, V., J.F. Schweigert, and D. Fournier. 1988. Stock assessments for British Columbia herring in 1987 and forecasts of the potential catch in 1988. Can. Tech. Rep. Fish. Aquat. Sci. 1990. 63 pp.

Hay, D.E. 1985. Reproductive biology of Pacific herring (Clupea harengus pallasi). Can. J. Fish. Aquat. Sci. 42(Suppl. 1):111-126.

Hourston, A.S. 1980. The decline and recovery of Canada's Pacific herring stocks. Rapp. P.-V. Reun. Cons. Int. Explor. Mer 177:143-153.

Otter Research Ltd. 1992. AUTODIF: A C++array extension with automatic differentiation for use in nonlinear modeling and statistics. Otter Research Ltd., Nanaimo, B.C.

Richards, L.J., J.T. Schnute, and N. Olsen. 1997. Visualizing catch-age analysis: A case study. Can. J. Fish. Aquat. Sci. 54:1646-1658.

Schweigert, J.F. 1993. Review and evaluation of methodology for estimating Pacific herring egg deposition. Bull. Mar. Sci. 53(2):818-841.

Schweigert, J.F., and M. Stocker. 1988. A new method for estimating Pacific herring stock size from spawn survey data and its management implications. N. Am. J. Fish. Manage. 8:63-74.

Schweigert, J.F., C. Fort, and L. Hamer. 1997. Stock assessments for British Columbia herring in 1996 and forecasts of the potential catch in 1997. Can. Tech. Rep. Fish. Aquat. Sci. 2173.73 pp.

Stevenson, C.J. 1954. The movement of herring in British Columbia waters as determined by tagging, with a description of tag and tag recovery methods. In: Herring tagging and results. Cons. Int. Explor. Mer Spec. Sci. Meeting 55. 36 pp. 
